\title{
Methods of diffusion-weighted and functional magnetic resonance imaging investigated in the human brain at ultra-high-field
}

Citation for published version (APA):

Seehaus, A. (2016). Methods of diffusion-weighted and functional magnetic resonance imaging investigated in the human brain at ultra-high-field. [Doctoral Thesis, Maastricht University]. Maastricht University. https://doi.org/10.26481/dis.20160928as

Document status and date:

Published: 01/01/2016

DOI:

10.26481/dis.20160928as

Document Version:

Publisher's PDF, also known as Version of record

Please check the document version of this publication:

- A submitted manuscript is the version of the article upon submission and before peer-review. There can be important differences between the submitted version and the official published version of record.

People interested in the research are advised to contact the author for the final version of the publication, or visit the DOI to the publisher's website.

- The final author version and the galley proof are versions of the publication after peer review.

- The final published version features the final layout of the paper including the volume, issue and page numbers.

Link to publication

\footnotetext{
General rights rights.

- You may freely distribute the URL identifying the publication in the public portal. please follow below link for the End User Agreement:

www.umlib.nl/taverne-license

Take down policy

If you believe that this document breaches copyright please contact us at:

repository@maastrichtuniversity.nl

providing details and we will investigate your claim.
}

Copyright and moral rights for the publications made accessible in the public portal are retained by the authors and/or other copyright owners and it is a condition of accessing publications that users recognise and abide by the legal requirements associated with these

- Users may download and print one copy of any publication from the public portal for the purpose of private study or research.

- You may not further distribute the material or use it for any profit-making activity or commercial gain

If the publication is distributed under the terms of Article $25 \mathrm{fa}$ of the Dutch Copyright Act, indicated by the "Taverne" license above, 
Arne Seehaus

Methods of diffusion-weighted and functional magnetic resonance imaging

Investigated in the human brain at ultra-high-field

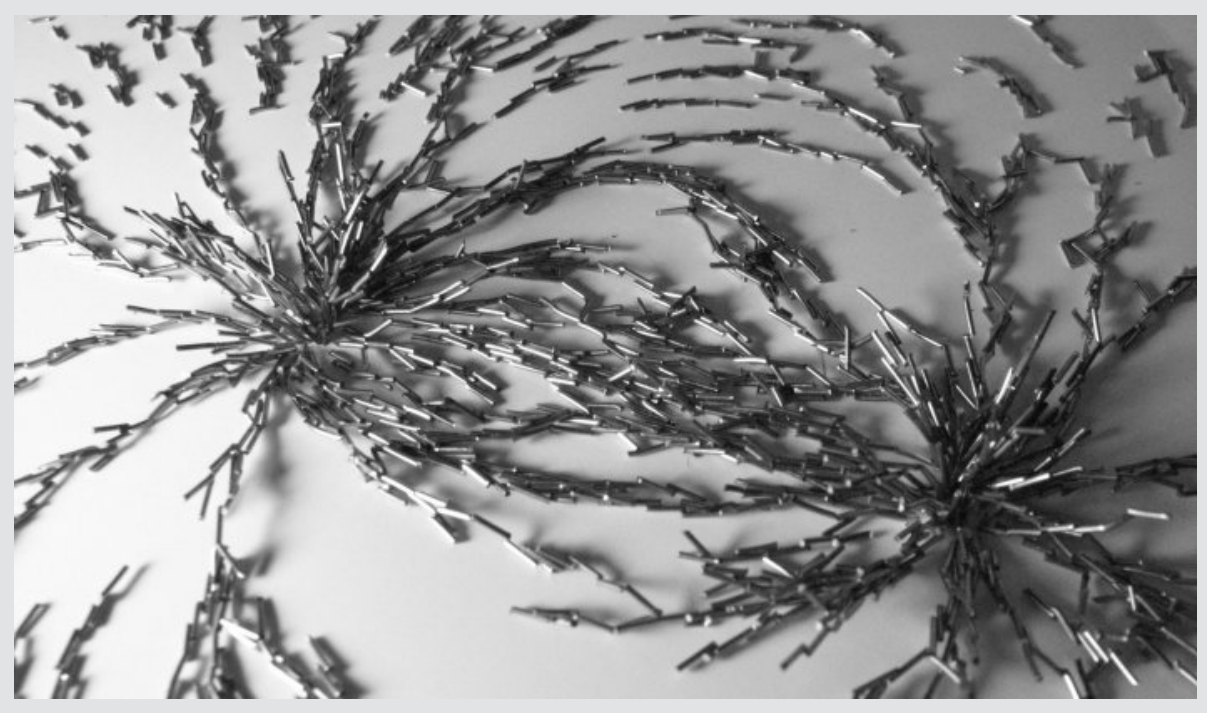



Methods of diffusion-weighted and functional magnetic resonance imaging investigated in the human brain at ultra-high-field

\section{DisSERTATION}

to obtain the degree of Doctor at the Maastricht University, on the authority of the Rector Magnificus, Prof. dr. Rianne M. Letschert, in accordance with the decision of the Board of Deans, to be defended in public on Wednesday, September 28, 2016, at 16:00 hours by Arne Konstantin Seehaus 
Supervisor:

Prof. dr. R. Goebel

Co-supervisors:

Dr. A. Roebroeck

Dr. J. Peters

Assessment Committee:

Prof. dr. E. Formisano (Chairman)

Dr. B. Jans,

Dr. P. Pullens, Antwerp University Hospital \& University of Antwerp Prof. dr. M. Wibral, J. W. Goethe University Frankfurt a. M.

Funding: Parts of this work were funded by the European Research Council (ERC-2010-AdG).

Cover picture: Windell Oskay (CC By 2.0, https://creativecommons.org/licenses/by/2.0/\#, picture detail)

(c) Arne Seehaus, 2016. 


\section{Contents}

I General Introduction 1

1 Preface ........................ 2

2 MRI physics ................... 3

3 Diffusion MRI .................. 4

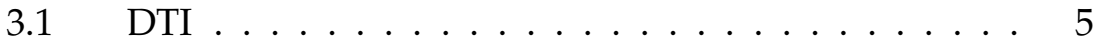

3.2 Alternative dMRI models . . . . . . . . . . . 7

4 DTI validation . . . . . . . . . . . . . . 8

4.1 Tractography validation ............ 9

4.2 Diffusion tensor validation . . . . . . . . . . 9

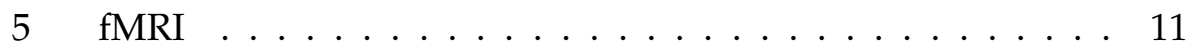

5.1 Methodological concerns . . . . . . . . . 12

5.2 fMRI statistics . . . . . . . . . . . . . 13

5.3 Cortical columns . . . . . . . . . . . . . 15

5.4 Radial bias in fMRI orientation decoding . . . . . . 17

II Histological validation of DTI: Tractography 25

1 Introduction . . . . . . . . . . . . . . . 26

2 Materials and Methods . . . . . . . . . . . . . . . . . . 29

2.1 Tissue . . . . . . . . . . . . . . . . . . . . 29

2.2 Carbocyanine tracing . . . . . . . . . . . . . 29

2.3 Diffusion MRI . . . . . . . . . . . . . . . . 30

2.4 DTI tractography . . . . . . . . . . . . 31

2.5 Alignment . . . . . . . . . . . . 32

2.6 Sensitivity and specificity analysis . . . . . . . 34

2.7 Entire tract analysis . . . . . . . . . . . . 36

3 Results .................... 37

3.1 Sensitivity/specificity . . . . . . . . 37 


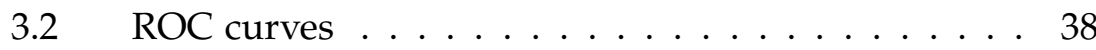

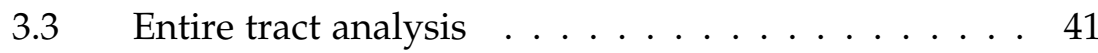

4 Discussion . . . . . . . . . . . . . . 41

$4.1 \quad$ DTI tractography . . . . . . . . . . . 41

4.2 Evaluation method . . . . . . . . . . . . . 46

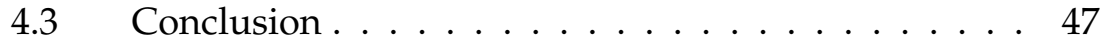

III Histological validation of DTI: Diffusion Tensors 53

1 Introduction . . . . . . . . . . . . . . . . . 54

2 Materials and Methods . . . . . . . . . . . . . . . 57

2.1 Tissue acquisition . . . . . . . . . . . . 57

2.2 Diffusion MRI . . . . . . . . . . . . . 58

2.3 Histological processing . . . . . . . . . . 58

2.4 Histological orientation analysis . . . . . . . . . . . 59

2.5 Alignment . . . . . . . . . . . . . . . 62

2.6 Diffusion tensor orientations . . . . . . . . . 63

$2.7 \quad$ Statistical evaluation . . . . . . . . . . . . . . 64

3 Results ........................ 65

3.1 Sample sizes . . . . . . . . . . . . 65

3.2 Orientation differences . . . . . . . . . . 65

3.3 Correlation and regression results . . . . . . . . 67

4 Discussion . . . . . . . . . . . . . . . 71

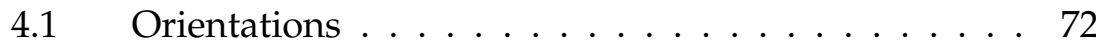

4.2 Scalar measures . . . . . . . . . . . . . . 75

4.3 Conclusion . . . . . . . . . . . . . . . 77

IV Sources of orientation decoding in 7 T fMRI data 85

1 Introduction . . . . . . . . . . . . . . . . 86

2 Materials and Methods . . . . . . . . . . . . . . . 92

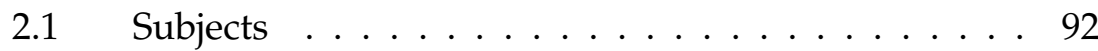

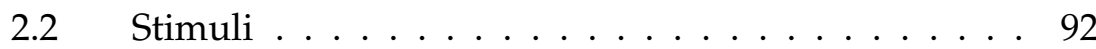

2.3 MRI acquisition . . . . . . . . . . . . . . . . 94

2.4 Analysis . . . . . . . . . . . . . . . . . . 94

3 Results ........................ 99 
3.1 Orientation preference shows radial bias . . . . . . 99

3.2 Orientation can be robustly decoded within visual field wedges . . . . . . . . . . . . . . . 999

3.3 Radial bias is strong for orientation preference, weak for classification performance . . . . . . . . . . . 100

3.4 Border information is not necessary for orientation decoding . . . . . . . . . . . . . . 101

3.5 Data normalization has no effect on classification performance . . . . . . . . . . . . . . . 101

3.6 Flow field stimulation evokes slightly radially biased response with inverse sign . . . . . . . . . . 102

4 Discussion . . . . . . . . . . . . . . . . . 102

V General discussion $\quad 113$

1 Histological validation of DTI tractography . . . . . . . . 114

2 Histological validation of diffusion tensors . . . . . . . . 116

3 Intermediate conclusion . . . . . . . . . . . . . . . . 118

4 Radial bias in fMRI orientation decoding . . . . . . . . . . 119

5 Conclusion . . . . . . . . . . . . . . . . . . . . . 121

$\begin{array}{ll}\text { A Knowledge valorization } & 123\end{array}$

$\begin{array}{ll}\text { B Acknowledgements } & 129\end{array}$

$\begin{array}{ll}\text { C Curriculum vitae } & 131\end{array}$ 


\section{Abbreviations}

BOLD Blood oxygenation level dependent

dMRI Diffusion(-weighted) magnetic resonance imaging

DTI, DT Diffusion tensor imaging, diffusion tensor

FA Fractional anisotropy

(f)MRI (functional) magnetic resonance imaging

FOD Fiber orientation distribution

GM Gray matter

HRF Hemodynamic response function

MD Mean diffusivity

MVPA Multi-voxel pattern analysis

NMR Nuclear magnetic resonance

RD Radial diffusivity

RF Radiofrequency

ROC Receiver operating characteristic

ROI Region of interest

ST Structure tensor

SVM Support vector machine

TE Echo time

TR Repetition time

WM White matter 
Chapter I

\section{General Introduction}




\section{Preface}

Nuclear magnetic resonance (NMR) is the physical phenomenon that molecules in a magnetic field can be excited by radio waves and reemit electromagnetic radiation while returning to their original state. This effect was first described in 1938 and successively became the basis for the spectroscopic analysis of substances over the following decades. In the early 1970s, it was reasoned that NMR might have practical applications not only in chemistry but also in biomedicine. This idea sparked the ingenious effort of several researchers to construct an NMR-based device to scan biological tissue and eventually led to the first magnetic resonance image of a human thorax in 1977 (Ai et al. 2012). Since then, magnetic resonance imaging (MRI) has constantly advanced and today is undoubtedly one of the most important imaging techniques in medicine and neuroscience.

The following manuscript is concerned with the methodological foundations of MRI. More precisely, I will investigate the validity of two different MRI methods, aiming to shed light on the relation between results from these methods and the underlying brain structure and function.

The first of the studied methods is diffusion tensor imaging (DTI), a technique to model the fiber architecture of the brain. In two subsequent studies, I compared DTI results to histological data obtained from human brain tissue. These studies are the subject of chapters 2 and 3. The second method I examined was multi-voxel pattern analysis (MVPA) of functional MRI data, which was used to investigate patterns of orientation tuning in the visual cortex. This project is portrayed in chapter 4 . In the following general introduction, I will review the theoretical principles behind each of those techniques, briefly describe the conducted studies and put them in a broader context. In the general discussion following the individual chapters, I will summarize and critically reassess my findings and speculate about future directions of diffusion imaging and MVPA, particularly classifier studies. 


\section{MRI physics}

First of all, I want to give a brief introduction into the physical principles of MRI (detailed introductions are provided in many textbooks, e.g. Bushong and Clarke 2013). The fundament of an MRI scanner is a strong magnetic field $\mathrm{B}_{0}$, which is created by a big coil kept at temperatures near absolute zero in order to be superconductant. It reaches field strengths in the order of a few Tesla. For comparison, the strongest permanent magnets in use have surface field strengths of about $1 \mathrm{~T}$ as well. Therefore, the key feature of the MRI magnetic field is not only its strength but also its homogeneity and its remarkable size allowing to place a human subject inside. While the most widely used clinical scanners operate at $1.5 \mathrm{~T}$, in research higher field strengths are common. Here, $3 \mathrm{~T}$ is referred to as high-field and everything above that as ultra-high-field.

The magnetic force exerted by $\mathrm{B}_{0}$ causes nuclei with a magnetic momentum (i.e. with nonzero spin) to align with the direction of the magnetic field, which is defined as longitudinal. From this orientation, the nuclei are temporarily forced away through exposition to a radiofrequency (RF) pulse matched to their precession frequency. This frequency is called Larmor frequency and depends on the type of nucleus and the magnetic field strength. In biomedical imaging, the nuclei of interest are protons due to their vast abundance in biological tissue. After excitation through the RF pulse, the protons form a magnetization vector orthogonal to the base magnetization, which can be measured by a receive coil. The rate at which the protons' longitudinal magnetization is restored $\left(\mathrm{T}_{1}\right)$ and at which the orthogonal magnetization decays $\left(T_{2}, T_{2}^{*}\right)$ is specific for different substances and can be used to obtain image contrasts.

To obtain a $\mathrm{T}_{1}$ contrast differentiating between two substances $\mathrm{A}$ and $\mathrm{B}$, the time between subsequent excitation pulses (called repetition time, TR) is chosen such that the longitudinal magnetization of substance A has already been reasonably restored at the time of the next RF pulse while the magnetization of B has not. As a result, A will be fully excitable 
again, yielding a strong signal, whereas the signal of B will be attenuated. Alternatively, the time between excitation and readout (echo time, TE) can be chosen such that only the transverse magnetization of one of the substances has remarkably decayed at time of readout, resulting in a smaller signal. Without further action, this yields a $\mathrm{T}_{2}^{*}$ contrast, which is sensitive to all sources of magnetization decay. If one is interested in only those sources of decay that happen due to molecular interactions and not due to fixed external sources such as magnetic field inhomogeneities ( $T_{2}$ contrast), then additionally a refocusing pulse is added in between excitation and readout. It results in a neutralization of all fixed effects.

Finally, by adding different magnetic field gradients on top of the base magnetization during excitation, readout and in between both steps, the specificity of NMR to the correct Larmor frequency is utilized to localize signals and create $3 \mathrm{D}$ images from these contrasts. Both $\mathrm{T}_{1}$ and $\mathrm{T}_{2}$ contrasts are capable to discern between different types of human tissue (Nitz and Reimer 1999), which gives them a wide range of applications in clinical practice and in neuroscience.

\section{Diffusion MRI}

Both a $T_{1}$ and a $T_{2}$ contrast measure static properties of molecules, roughly speaking the amount of interaction of proton spins with the magnetic field or with each other. Besides that, MRI can be made sensitive to molecular motion as well. In diffusion imaging (dMRI), an additional diffusion gradient is applied twice, once between the excitation and the refocusing pulse, and once between refocusing pulse and readout (Bernstein et al. 2004, pp 274f). As a consequence, the gradients cancel each other out for all proton populations except the ones diffusing along the gradient direction. For those, the rephasing process initiated by the refocusing pulse is disrupted, resulting in signal attenuation. By this principle, a diffusion contrast for a certain direction in space is obtained. Combining 
measurements of multiple different diffusion directions, the entire 3D diffusivity profile within a tissue probe can be sampled.

This is particularly interesting in neuroscience because nerve fibers due to their elongated shape and their lipid membranes constrain water diffusion remarkably. For this reason, dMRI promises to provide information about fiber architecture at a spatial scale much smaller than the actual voxel size. This however, is an indirect way of information acquisition. It needs mathematical models first to aggregate the individual maps for the different diffusion directions into structures representing the whole 3D diffusivity and second to obtain the sought-after information from these structures. Among the most relevant information are overall diffusivity and diffusion anisotropy within voxels as well as the course of fiber pathways across voxels.

\subsection{DTI}

The simplest dMRI model is diffusion tensor imaging, which has first been described in Basser et al. (1994): The namesake diffusion tensor is a symmetric $3 \times 3$ matrix $D$, which reflects the linear relation between proton concentration gradient $\nabla C$ and flux $J$ via the formula

$$
J=-D \nabla C
$$

The diffusion tensor conveys information about the physical structure inside an imaging voxel: If there are no structures present which hinder water from flowing, then a concentration gradient in any direction is directly translated into a corresponding flux, so $D$ (ideally) is a multiple of the identity matrix. If on the contrary, there are obstacles such as lipid membranes, then concentration gradients in certain directions cause less flux than others, and a gradient in one direction can cause flux in another direction. This principle does not only hold for directed flow, but for diffusion processes as well. Therefore, $D$ can be estimated by probing the amount of signal attenuation caused by different diffusion gradients. This 
is done on the basis of the formula

$$
\ln \frac{S}{S_{0}}=-\sum_{i=1}^{3} \sum_{j=1}^{3} b_{i j} D_{i j}
$$

where $S$ is the signal in a voxel with, $S_{0}$ without application of the diffusion gradient. The b-matrix $\left(b_{i j}\right)$ provides information about the contribution of each entry of $D$ towards the signal attenuation and is essentially a function of the diffusion gradient. The b-matrices corresponding to different diffusion gradients along with the measured signal attenuation values yield a system of linear equations which can be solved for $D$. From there, the first analysis step is usually to obtain the eigenvectors and eigenvalues of $D$. The eigenvector $v_{1}$ corresponding to the largest eigenvalue $\lambda_{1}$ is interpreted as the primary axis of diffusion. The absolute and relative magnitudes of the eigenvalues contain information about the overall diffusivity in a voxel and about diffusion anisotropy. These are most often quantified in terms of mean diffusivity (MD) and fractional anisotropy (FA) (Basser and Pierpaoli 1996), following the formulae

$$
\begin{aligned}
M D & =\bar{\lambda}=\frac{\lambda_{1}+\lambda_{2}+\lambda_{3}}{3} \\
F A & =\sqrt{\frac{3\left(\left(\lambda_{1}-\bar{\lambda}\right)^{2}+\left(\lambda_{2}-\bar{\lambda}\right)^{2}+\left(\lambda_{3}-\bar{\lambda}\right)^{2}\right)}{2\left(\lambda_{1}^{2}+\lambda_{2}^{2}+\lambda_{3}^{2}\right)}}
\end{aligned}
$$

Furthermore, $\lambda_{1}$ and the average $\left(\lambda_{2}+\lambda_{3}\right) / 2$ of the two minor eigenvalues are often termed axial diffusivity (AD) and radial diffusivity (RD), respectively. This nomenclature refers to the typical illustration of diffusion tensors as ellipsoids (Basser 1995).

In a secondary step, fiber pathways can be modeled in a procedure referred to as tractography. A tractography algorithm typically models a fiber path as a curve in 3D space and interprets the calculated principal diffusion directions as samples of the curve's tangents. Mathematically, this problem translates to the solution of a differential equation. Its initial condition is an arbitrarily chosen "seed point" on the fiber tract. Other parameters of the algorithm are different stopping criteria, such as 
maximum angles between neighboring diffusion vectors and minimal FA values (Basser et al. 2000).

\subsection{Alternative dMRI models}

The possibilities of DTI are rich and it sees a wide range of clinical as well as research applications (a number of examples are given in chapter 2). However, due to its simplicity, DTI faces certain limitations. While modeling one primary diffusion direction per voxel suffices to describe unidirectional fiber pathways, more complex fiber architecture can not be grasped with diffusion tensors. Particularly, the model cannot discern between no fiber structure present at all, crossing or curving fiber tracts or fiber populations with orientations uniformly distributed in space (Roebroeck et al. 2008). To be able to distinguish these cases, more sophisticated models are needed. Examples for such models are q-ball imaging (Tuch 2004), diffusion spectrum imaging (Wedeen et al. 2005) or spherical deconvolution (Tournier et al. 2004). In one way or another, these models attempt to represent the entire 3D diffusion profile instead of projecting it onto the coordinate axes.

Each diffusion model has its distinct advantages and disadvantages. Generally, DTI has a limited scope but yields very robust data within its scope. Complex models have more degrees of freedom and are therefore more powerful but also more prone to noise, since it gets incorporated in the model more easily, a problem known as overfitting (Jones et al. 2013). Also, these models require imaging protocols with many different acquired diffusion directions, termed high angular resolution diffusion imaging (HARDI). While the estimation of diffusion tensors profits from a high angular resolution as well, DTI can operate on a minimum of merely six different diffusion gradients, allowing for relatively short scanning times. 


\section{DTI validation}

The previous section illustrates that in practice, evaluating the suitability of a diffusion model for a given problem and likewise identifying the optimal model is not at all trivial. For this reason, it is desirable to collect information about the performance of diffusion models in real-world applications in order to complement the theoretical considerations made above. This endeavor is the subject of validation studies. Concretely, a validation study of DTI should answer some of the following questions: Does DTI accurately describe fiber architecture where it is theoretically expected to work well (in the presence of unidirectional fiber bundles)? How strongly is DTI affected by its limitations in practice and what precisely happens in cases which cannot be sufficiently modeled? How are DTI results related to anatomical characteristics? And lastly, which secondary measures and which choice of parameters optimally reflect these relations?

There has been a considerable number of previous validation studies, the methods of which are reviewed in chapters 2 and 3. One possible approach is to acquire a diffusion scan of an ex-vivo tissue probe, section it, treat it with some kind of staining in order to highlight specific anatomical characteristics and compare those to the dMRI data. Among the various validation methods, such histological analyses are often seen as the gold standard since they provide the closest approximation of the real situation faced when scanning live subjects.

While some histological validation studies of DTI had already been published when I started my work on this topic, they had all been conducted on animals (Lin et al. 2001, Kaufman et al. 2005, Leergaard et al. 2010). A direct comparison between human brain sections and DTI volumes acquired from the same tissue probe was at that time missing. To this end, we conducted two studies in which we investigated DTI on two different conceptual levels with histological analyses of human brain tissue. 


\subsection{Tractography validation}

In the first study, we tested the validity of modeled fiber tracts. Our main question was how sensitively and how specificly area connections are modeled by means of DTI tractography. That is, we aimed to quantify how likely two points that are connected via nerve fibers are also connected via a modeled fiber tract and vice versa. The main challenge in obtaining this kind of information is the obligatory sectioning of the tissue which cuts all connections in one space dimension. While reconstructions of 3D volumes from stained sections are possible on a macroscopic level (Amunts et al. 2013), the microscopic reconstruction of axons is only possible for very limited numbers of individually stained exemplars. We were able to circumvent this problem by utilizing histological tracers. These are substances which are injected into a piece of tissue and diffuse along nerve fibers. Here, the stained fiber parts, without any 3D reconstruction, carry information about connectivity across sections, at least with respect to the injection site. By performing tractography originating from stained and unstained locations and testing whether the modeled fiber tracts reach the injection site, we managed to obtain numbers of true and false positives and negatives, as needed for the target signal detection analysis.

As the main results of this study, we found that fiber connections modeled based on DTI were highly sensitive and specific for some but not all tested fiber populations, depending on whether the populations followed an unidirectional pattern or not. Furthermore, we were able to report optimal tractography parameters with respect to the sensitivity/specificity tradeoff.

\subsection{Diffusion tensor validation}

The aim of the second study was a validation of the diffusion tensors themselves at a high spatial resolution. We investigated how well their primary diffusion directions align with fiber orientations and how derived measures of anisotropy and overall diffusivity are correlated to fiber archi- 
tecture both qualitatively and quantitatively. Since this was an analysis within voxels, we did not need any information about fiber connections. This allowed us to employ a staining method that globally stains fiber material and thus provides a much larger sample size than the tracers used in the first project. The challenge in this study was to develop an algorithm to automatically obtain fiber orientations from the stained sections digitized at microscopic resolution. In chapter 3, I will shortly review different approaches towards such an algorithm. We decided to employ structure tensors since these are linear structures based on image gradients and thus of all available methods showed the best structural resemblance with diffusion tensors. We conducted the structure tensor analysis per image pixel and aggregated the results over areas equaling the voxel size in the imaging experiment. This yielded 2D fiber orientation distributions (FOD) which we compared to the diffusion tensors projected into the sectioning planes.

The comparison was made on two levels: First, the agreement between principal diffusion and fiber orientations was determined. Second, FA, $\mathrm{MD}$ and RD in the DTI volume were correlated with staining intensity (as an indirect measure of myelinization) and the parameters of the model fitted to the histological FODs.

Our main finding was a that DTI behaved exactly as expected in white matter: Prominent unidirectional fiber pathways were modeled with high accuracy, whereas at fiber crossings, diffusion tensor directions resembled averages of the different fiber orientations present. In total, diffusion tensors with a very precise goodness-of-fit outnumbered the weakly matching ones. Even in the deeper gray matter layers, DTI still seemed to perform sufficiently well. In the middle and superficial layers, the myelin staining we used gave an incomplete image of the largely unmyelinated fiber architecture. With respect to the scalar measures, we found FA to be correlated with unimodal FODs, strong staining (indicating prominent fiber pathways) and well matching principal directions. The opposite was the case for MD and RD. MD showed a particularly strong negative 
correlation with staining intensity which makes sense since diffusivity is generally hindered by the presence of myelin sheaths.

We also investigated $\mathrm{AD}$ in the same fashion as the other secondary DTI measures, but we found no comparably strong relations with histological measures. We attribute this to the fact that AD only contains the information of one of the three DT eigenvalues, whereas RD includes two, FA and MD all three eigenvalues. Therefore, AD should pose the least robust of these measures and we would as a consequence not recommend its usage. For the publication, these considerations were omitted due to space limitations.

\section{5 fMRI}

All MRI methods discussed so far were structural: They provide images of the physical structure of an object, whether in form of a $T_{1}, T_{2}$ or a diffusion contrast. On the contrary, functional magnetic resonance imaging (fMRI) provides information about dynamic processes in the brain through acquisition of multiple MRI volumes in rapid succession while exposing test subjects to some kind of psychological experiment. In most cases, the physiological basis of $\mathrm{fMRI}$ is the susceptibility of $\mathrm{T}_{2}^{*}$ contrasts to blood oxygenation. This is due to the fact that oxyhemoglobin being diamagnetic and deoxyhemoglobin being paramagnetic show different rates of dephasing and thus different $\mathrm{T}_{2}^{*}$ values. Utilizing this relation, one can obtain a blood oxygenation level dependent (BOLD-) contrast by comparing $\mathrm{T}_{2}^{*}$-images in response to different experimental conditions (Ogawa et al. 1990, Bandettini et al. 1992). The hemodynamic information obtained in this way can be interpreted in terms of neuronal activity, since both are known to be connected from earlier studies (Roland et al. 1980, Logothetis et al. 2001). 


\subsection{Methodological concerns}

As with diffusion MRI, the study of brain activity through fMRI experiments is less straightforward than a simple anatomical scan but relies on different assumptions and intermediate data processing and modeling steps. Precisely, there are at least four conceptual steps between fMRI data and the cognitive phenomenon a researcher wants to investigate in terms of brain involvement:

First of all, the average percent signal change between an experimental and a control trial (a trial is defined as a single exposition to an experimental condition, typically for the duration of a few seconds) is usually small and gets surpassed by the inter-trial variability (Desmond and Glover 2002). Therefore, many trials are needed in an fMRI experiment, which have to be statistically aggregated in one way or another.

Second, the connection between a BOLD contrast and the causal hemodynamic processes is not trivial. There are at least three main contributors to this relation, regional cerebral blood flow, cerebral blood volume and the amount of oxygen consumption. They affect the BOLD response in different directions and with different temporal response functions. As a result, BOLD responses follow a specific temporal pattern which emerges from the interplay of these factors and which is termed hemodynamic response function (HRF) (Buxton et al. 2004). This function has to be adequately modeled and incorporated into statistical analyses, which is usually done by convolving boxplots of the experimental conditions with the HRF and using the resulting idealized time series to predict the empirical ones.

Third, one has to consider the exact relation between neuronal activity and hemodynamic response, a problem termed neurovascular coupling. That there is a relation was already assumed in the late 19th century (Roy and Sherrington 1890). Since then, there has been extensive research on this topic. One of the main findings so far is that synaptic activity, particularly the restoration of sodium gradients after excitation, is the most energy consuming process in neuronal activity and is most strongly corre- 
lated to hemodynamic responses (Jueptner and Weiller 1995, Buxton et al. 2004). Consequently, BOLD responses can be assumed to reflect primarily excitatory neuronal input activity. Nevertheless, a comprehensive model unifying the many aspects of neuronal activity and hemodynamics and connecting them is still lacking. As a consequence, in most applications one prototypical HRF is used in all brain areas. While this seems to suffice in practice, one has to keep in mind that there might be intricate differences to the HRF of different areas, depending on their exact neuroanatomical structure, their way of functioning, or their spatial relation with capillaries.

Lastly, the MRI environment is very limiting with regards to experimental design. Subjects have to reside motionless in a narrow tube, wear hearing protection and can usually only get visual stimulation via a small screen. Furthermore, no magnetically susceptible equipment may be used in vicinity to the MRI scanner. Consequently, methodological concerns about generalizing experimental results to real-world settings are particularly relevant for fMRI studies.

\section{2 fMRI statistics}

As it becomes evident from these considerations, there are many intricacies of fMRI still to be explored. In the second pillar of my work I was concerned with the first of the stages explained above, the statistical treatment of fMRI data. Classically, fMRI analyses are univariate: Each voxel represents a separate experiment where its activation time series is predicted by the experimental conditions. If the voxel response is remarkably stronger following one compared to another condition, then this will result in a high significance value for this voxel and the task processing will be regarded to take place in this location (Amaro and Barker 2006). Alternatively, statistics can be performed multivariately over an entire region of interest (ROI). One can for example, correlate activation patterns of voxel populations between different conditions (Kriegeskorte et al. 2008, Connolly et al. 2012) or train a classifier to distinguish between 
those patterns (Haxby et al. 2001, Kamitani and Tong 2005, Haynes and Rees 2005). Among the available classifiers, support vector machines (SVM) and discriminant analysis are most common and have been reported to show the highest performance (Misaki et al. 2010). All these multivariate approaches are subsumed under the label of multi-voxel pattern analysis (MVPA).

As will be discussed in more detail in chapter 4, the advantage of MVPA over univariate analyses is most importantly its high sensitivity. MVPA can often detect small condition differences which do not reach significancy in single voxels but which become apparent in the combined activation patterns. This can lead to mind-boggling results such as the decoding of imagined stimuli from fMRI data (Vetter et al. 2014, Emmerling et al. 2016). The cost of this high sensitivity is the spatial information: While the univariate approach can pin an effect down to those voxels where it was most pronounced, MVPAs only yield results for the entire ROI. There are approaches to circumvent this problem, such as operating on small individual ROIs, centered around each voxel (Kriegeskorte et al. 2006). However, the localization this yields is less precise and robust than in the univariate case where it is inherent (Etzel et al. 2013).

While MVPA results cannot be precisely located, an interesting question is whether any further information about the underlying voxel patterns can be inferred. That is, are MVPAs essentially black boxes translating ROI activitation patterns into correlations, performance values etc.? Or do they convey implicit information about how processes in the brain are organized, possibly even on a subvoxel level?

The problem here is the relative inaccessibility of the inner workings of particularly machine learning algorithms. Even though theoretically it is well known how they work, the high dimension of the feature space (i.e. the voxel count) makes it hard to study them in practice. Likewise, intermediate results of those algorithms, e.g. the support vectors selected by an SVM in the learning phase, are difficult to interpret. As a consequence, one has for the most part to rely on indirect approaches. One 
possibility is to form and test hypotheses about the effects of different kinds of data manipulation on the MVPA output. Although there have been very interesting attempts in this direction, the results are often hotly debated (Op de Beeck 2010b, Kamitani and Sawahata 2010). This might not only be caused by the intransparency of MVPAs, but also by the fact that these studies are often motivated by a neuroscientific paradigm which itself is quite controversial: The concept of the cortical column.

\subsection{Cortical columns}

The cortical column is a model of the elementary "building block" of the cortex. It is a hypothesized arrangement of neurons with similar function into a cylindrical structure which vertically extends through all cortical layers.

The first evidence for an organization of the cortex in cylindrical functional units was presented by Mountcastle et al. (1957). He penetrated cats' somatosensory cortices with long microelectrodes and found that neurons at different positions on the electrode responded to the same class of tactile stimuli when the electrode was inserted orthogonally into the cortex. On the contrary, at oblique penetration angles, the sampled neurons responded to different stimulus classes. With the same method, Hubel and Wiesel $(1962,1977)$ investigated the visual cortex of cat and monkey in an extensive series of studies which earned them a Nobel prize in 1981. They found two orthogonal patterns of columnar organization for ocular dominance and stimulus orientation, a system which was later termed "ice cube" model. These findings were very influential and promoted the view that the fundamental unit of cortical organization are said column-shaped arrangements of neurons sharing the same response properties (Szentagothai 1978, Mountcastle 1997). Findings of columnar patterns in the motor and auditory cortex complemented results from the visual and somatosensory domain (Asanuma 1975, Imig et al. 1977). In the last decade, fMRI advanced far enough that voxel sizes below one millimeter became possible. In succession, functional patterns in the human 
visual cortex similar to the columnar patterns found earlier were reported for ocular dominance (Yacoub et al. 2007), orientation (Yacoub et al. 2008) and axis of motion (Zimmermann et al. 2011).

Despite the high acceptance the column concept gained in the years after its introduction, it faced an increasing amount of criticism later on. The most important points are first the high variability of columnar organization. The shape, size and mere presence of columnar patterns varies remarkably between different related species as well as between and even within individuals of one species (Adams and Horton 2009). Also, there are cortical areas which are not organized in columns at all (Rockland 2010). Second, the term column suggests a structure which has the shape of a cylinder and is sharply delineated from neighboring columns. This perception however might be biased by the method of microelectrode penetration which is prone to yield such an organizational pattern. Studies employing different approaches give evidence that in general there are no abrupt borders between functional preference but rather smooth transitions (Horton and Adams 2005, Rockland 2010) and that depending on the cortical layer, the horizontal extent of a column varies (Boucsein et al. 2011). Last, in the classical point of view, the column is both a structural and a functional unit of cortical organization. Indeed, columnar structures have been shown both functionally (the studies reviewed above) as well as histologically in sections stained for cell bodies. However, both patterns seem to be unrelated (Horton and Adams 2005). The anatomical columns are considerably smaller than their functionally defined counterparts (about $50 \mu \mathrm{m}$ vs $300 \mu \mathrm{m}$ in diameter, hence often termed "microcolumns") and might arise due to developmental rather than functional factors (Purves et al. 1992). Furthermore, as seen in the ice cube model, there can be overlapping columnar patterns for different functional domains. The more such domains are mapped, the harder it is to define corresponding structural columns. As a consequence, the term column nowadays is still popular but often used in a weakened form where it refers to a functional unit on a scale of about $0.1-1 \mathrm{~mm}$, without claiming sharp borders or a strictly cylindrical shape. 


\subsection{Radial bias in fMRI orientation decoding}

The debate about the existence and relevance of cortical columns has pervaded up to now particularly in the context of MVPAs in the visual domain. Proponents of the hyperacuity hypothesis claim that orientation columns are the reason why the orientation of viewed grating patterns can be decoded from fMRI data (Kamitani and Tong 2005, Haynes and Rees 2005). Even when fMRI volumes are acquired at a voxel size larger than the size of a column, so the theory, the inhomogeneous distribution of columns and/or aliasing effects cause different voxels to exhibit different orientation preferences. The opposition to this view regards weak global organization patterns as responsible for orientation decodability (Op de Beeck 2010a, Freeman et al. 2011). A possible candidate for such a pattern is the radial bias: Orientations are best perceived when they are radial with respect to the point of fixation. This effect has been shown both behaviorally and in fMRI data (Sasaki et al. 2006) and might therefore be learned by a classifier algorithm.

The third study I conducted is concerned with both the methodological (how can classifiers be analyzed?) and the practical (how can orientation decoding be explained?) aspect of this problem. Reviewing the literature on the aforesaid debate, I particularly noticed two points in the initial paper by Kamitani and Tong (2005): First, the authors argue in favor of hyperacuity by stating that they found a scattered orientation preference map. Although they admit some spatial clustering, this was regarded as artifacts. This pattern, observed at a voxel size of $3 \mathrm{~mm}$, could indeed be the consequence of irregularly distributed orientation columns at a submillimeter scale. But it might just as well illustrate an organization pattern at a periodicity of a few millimeters, without any artifacts or partial volume effects. At submillimeter resolution, we should expect columnar, i.e. scattered, organization patterns according to Yacoub et al. (2008). However, it would be interesting to see what kind of pattern is observed at an intermediate resolution, as this could answer the question whether 
columnar patterns propagate with increasing voxel size or whether there are independent patterns at different spatial scales.

Second, the authors bring forward an argument against the radial bias as a source of decodable information: They state that data normalization within visual field wedges did not have a remarkable effect on performance, implying that this processing step removed the radial bias pattern from the data. Yet this is not the case. Accordingly, it has been reported in a more recent work that classifiers are generally invariant to data normalization (Misaki et al. 2010). However, the argument seems to have prevailed (Op de Beeck 2010a).

In order to address these points, I conducted a similar experiment at $7 \mathrm{~T}$ and a functional voxel size of $1.25 \mathrm{~mm}$. I showed the subjects oriented grating patterns and analyzed the BOLD responses in the visual cortex in terms of radial bias and orientation decodability. With respect to the first point, I visualized the strength of statistical condition contrasts and weighted support vectors, resulting from the classification task, on the cortical surface. The patterns I found were not scattered but showed a spatial frequency in the order of a few millimeters. The second point was addressed by showing the inability of data normalization to eliminate a radial bias pattern in simulated data. I proposed an improved normalization-based approach, which however did not show convincing results in practice either. In an alternative approach to evaluate the importance of the radial bias, I performed the entire classification on separate visual field wedges. The performance within wedges was poorer than in equally sized random subsets of the entire voxel population, but not by a large margin. From the different analyses conducted, I conclude that the radial bias does probably play a role in orientation decoding, but not a crucial one. While I could not identify the main source of information, the data speak for a mesoscale rather than a subvoxel pattern. Future directions to further illuminate this question are discussed in chapter 4 as well as the general discussion. 


\section{References}

Adams, D. L. and Horton, J. C. (2009). Ocular dominance columns: enigmas and challenges. The Neuroscientist, 15(1):62-77.

Ai, T., Morelli, J. N., Hu, X., Hao, D., Goerner, F. L., Ager, B., and Runge, V. M. (2012). A historical overview of magnetic resonance imaging, focusing on technological innovations. Investigative radiology, 47(12):725741.

Amaro, E. and Barker, G. J. (2006). Study design in fmri: basic principles. Brain and cognition, 60(3):220-232.

Amunts, K., Lepage, C., Borgeat, L., Mohlberg, H., Dickscheid, T., Rousseau, M.-É., Bludau, S., Bazin, P.-L., Lewis, L. B., Oros-Peusquens, A.-M., et al. (2013). BigBrain: an ultrahigh-resolution 3D human brain model. Science, 340(6139):1472-1475.

Asanuma, H. (1975). Recent developments in the study of the columnar arrangement of neurons within the motor cortex. Physiological reviews, 55(2):143-156.

Bandettini, P. A., Wong, E. C., Hinks, R. S., Tikofsky, R. S., and Hyde, J. S. (1992). Time course EPI of human brain function during task activation. Magnetic resonance in medicine, 25(2):390-397.

Basser, P., Mattiello, J., and LeBihan, D. (1994). Estimation of the effective self-diffusion tensor from the NMR spin echo. Journal of Magnetic Resonance, Series B, 103:247-254.

Basser, P., Pajevic, S., Pierpaoli, C., Duda, J., and Aldroubi, A. (2000). In vivo fiber tractography using DT-MRI data. Magnetic Resonance in Medicine, 44:625-632.

Basser, P. J. (1995). Inferring microstructural features and the physiological state of tissues from diffusion-weighted images. NMR in Biomedicine, 8(7):333-344. 
Basser, P. J. and Pierpaoli, C. (1996). Microstructural and physiological features of tissues elucidated by quantitative-diffusion-tensor MRI. Journal of magnetic resonance, 111:209-219.

Bernstein, M. A., King, K. F., and Zhou, X. J. (2004). Handbook of MRI pulse sequences. Elsevier.

Boucsein, C., Nawrot, M., Schnepel, P., and Aertsen, A. (2011). Beyond the cortical column: abundance and physiology of horizontal connections imply a strong role for inputs from the surround. Frontiers in Neuroscience, 5:32.

Bushong, S. C. and Clarke, G. (2013). Magnetic resonance imaging: physical and biological principles. Elsevier Health Sciences.

Buxton, R. B., Uludağ, K., Dubowitz, D. J., and Liu, T. T. (2004). Modeling the hemodynamic response to brain activation. NeuroImage, 23:220-233.

Connolly, A. C., Guntupalli, J. S., Gors, J., Hanke, M., Halchenko, Y. O., Wu, Y.-C., Abdi, H., and Haxby, J. V. (2012). The representation of biological classes in the human brain. The Journal of Neuroscience, 32(8):2608-2618.

Desmond, J. E. and Glover, G. H. (2002). Estimating sample size in functional MRI (fMRI) neuroimaging studies: statistical power analyses. Journal of neuroscience methods, 118(2):115-128.

Emmerling, T. C., Zimmermann, J., Sorger, B., Frost, M. A., and Goebel, R. (2016). Decoding the direction of imagined visual motion using $7 \mathrm{~T}$ ultra-high field fMRI. NeuroImage, 125:61-73.

Etzel, J. A., Zacks, J. M., and Braver, T. S. (2013). Searchlight analysis: promise, pitfalls, and potential. Neuroimage, 78:261-269.

Freeman, J., Brouwer, G. J., Heeger, D. J., and Merriam, E. P. (2011). Orientation decoding depends on maps, not columns. The Journal of Neuroscience, 31(13):4792-4804.

Haxby, J. V., Gobbini, M. I., Furey, M. L., Ishai, A., Schouten, J. L., and 
Pietrini, P. (2001). Distributed and overlapping representations of faces and objects in ventral temporal cortex. Science, 293(5539):2425-2430.

Haynes, J.-D. and Rees, G. (2005). Predicting the orientation of invisible stimuli from activity in human primary visual cortex. Nature neuroscience, 8(5):686-691.

Horton, J. C. and Adams, D. L. (2005). The cortical column: a structure without a function. Philosophical Transactions of the Royal Society of London B: Biological Sciences, 360(1456):837-862.

Hubel, D. H. and Wiesel, T. N. (1962). Receptive fields, binocular interaction and functional architecture in the cat's visual cortex. The Journal of physiology, 160(1):106-154.

Hubel, D. H. and Wiesel, T. N. (1977). Ferrier lecture: Functional architecture of macaque monkey visual cortex. Proceedings of the Royal Society of London B: Biological Sciences, 198(1130):1-59.

Imig, T., Adria, H., et al. (1977). Binaural columns in the primary field (A1) of cat auditory cortex. Brain research, 138(2):241-257.

Jones, D. K., Knösche, T. R., and Turner, R. (2013). White matter integrity, fiber count, and other fallacies: The do's and don'ts of diffusion MRI. NeuroImage, 73:239-254.

Jueptner, M. and Weiller, C. (1995). Review: does measurement of regional cerebral blood flow reflect synaptic activity?-implications for pet and fmri. NeuroImage, 2(2PA):148-156.

Kamitani, Y. and Sawahata, Y. (2010). Spatial smoothing hurts localization but not information: pitfalls for brain mappers. NeuroImage, 49(3):19491952.

Kamitani, Y. and Tong, F. (2005). Decoding the visual and subjective contents of the human brain. Nature neuroscience, 8(5):679-685.

Kaufman, J., Ahrens, E., Laidlaw, D., Zhang, S., and Allman, J. (2005). Anatomical analysis of an aye-aye brain (Daubentonia madagascariensis, 
primates: Prosimii) combining histology, structural magnetic resonance imaging, and diffusion-tensor imaging. The Anatomical Record, Part A: Discoveries in Molecular, Cellular, and Evolutionary Biology, 287:1026-1037.

Kriegeskorte, N., Goebel, R., and Bandettini, P. (2006). Information-based functional brain mapping. Proceedings of the National Academy of Sciences of the United States of America, 103(10):3863-3868.

Kriegeskorte, N., Mur, M., and Bandettini, P. A. (2008). Representational similarity analysis-connecting the branches of systems neuroscience. Frontiers in systems neuroscience, 2:4.

Leergaard, T., White, N., de Crespigny, A., Bolstad, I., D'Arceuil, H., Bjaalie, J., and Dale, A. (2010). Quantitative histological validation of diffusion MRI fiber orientation distributions in the rat brain. PLoS One, 5:e8595.

Lin, C., Tseng, W., Cheng, H., and Chen, J. (2001). Validation of diffusion tensor magnetic resonance axonal fiber imaging with registered manganese-enhanced optic tracts. NeuroImage, 14:1035-1047.

Logothetis, N. K., Pauls, J., Augath, M., Trinath, T., and Oeltermann, A. (2001). Neurophysiological investigation of the basis of the fMRI signal. Nature, 412(6843):150-157.

Misaki, M., Kim, Y., Bandettini, P. A., and Kriegeskorte, N. (2010). Comparison of multivariate classifiers and response normalizations for patterninformation fMRI. NeuroImage, 53(1):103-118.

Mountcastle, V. B. (1997). The columnar organization of the neocortex. Brain, 120(4):701-722.

Mountcastle, V. B. et al. (1957). Modality and topographic properties of single neurons of cat's somatic sensory cortex. J neurophysiol, 20(4):408434.

Nitz, W. and Reimer, P. (1999). Contrast mechanisms in MR imaging. European radiology, 9(6):1032-1046. 
Ogawa, S., Lee, T.-M., Kay, A. R., and Tank, D. W. (1990). Brain magnetic resonance imaging with contrast dependent on blood oxygenation. Proceedings of the National Academy of Sciences, 87(24):9868-9872.

Op de Beeck, H. P. (2010a). Against hyperacuity in brain reading: spatial smoothing does not hurt multivariate fMRI analyses? Neurolmage, 49(3):1943-1948.

Op de Beeck, H. P. (2010b). Probing the mysterious underpinnings of multi-voxel fMRI analyses. NeuroImage, 50(2):567-571.

Purves, D., Riddle, D., and LaMantia, A. (1992). Iterated patterns of brain circuitry (or how the cortex gets its spots). Trends in neurosciences, 15(10):362-368.

Rockland, K. S. (2010). Five points on columns. The neocortical column, page 6 .

Roebroeck, A., Galuske, R., Formisano, E., Chiry, O., Bratzke, H., Ronen, I., Kim, D., and Goebel, R. (2008). High-resolution diffusion tensor imaging and tractography of the human optic chiasm at 9.4 T. NeuroImage, 39:157168.

Roland, P., Skinhoj, E., Lassen, N., and Larsen, B. (1980). Different cortical areas in man in organization of voluntary movements in extrapersonal space. Journal of Neurophysiology, 43(1):137-150.

Roy, C. S. and Sherrington, C. S. (1890). On the regulation of the bloodsupply of the brain. The Journal of physiology, 11(1-2):85.

Sasaki, Y., Rajimehr, R., Kim, B. W., Ekstrom, L. B., Vanduffel, W., and Tootell, R. B. (2006). The radial bias: a different slant on visual orientation sensitivity in human and nonhuman primates. Neuron, 51(5):661670.

Szentagothai, J. (1978). The ferrier lecture, 1977: the neuron network of the cerebral cortex: a functional interpretation. Proceedings of the Royal Society of London B: Biological Sciences, 201(1144):219-248. 
Tournier, J.-D., Calamante, F., Gadian, D. G., and Connelly, A. (2004). Direct estimation of the fiber orientation density function from diffusionweighted MRI data using spherical deconvolution. NeuroImage, 23(3):1176-1185.

Tuch, D. S. (2004). Q-ball imaging. Magnetic Resonance in Medicine, 52(6):1358-1372.

Vetter, P., Smith, F. W., and Muckli, L. (2014). Decoding sound and imagery content in early visual cortex. Current Biology, 24(11):1256-1262.

Wedeen, V. J., Hagmann, P., Tseng, W.-Y. I., Reese, T. G., and Weisskoff, R. M. (2005). Mapping complex tissue architecture with diffusion spectrum magnetic resonance imaging. Magnetic Resonance in Medicine, 54(6):1377-1386.

Yacoub, E., Harel, N., and Uğurbil, K. (2008). High-field fMRI unveils orientation columns in humans. Proceedings of the National Academy of Sciences, 105(30):10607-10612.

Yacoub, E., Shmuel, A., Logothetis, N., and Uğurbil, K. (2007). Robust detection of ocular dominance columns in humans using Hahn spin echo BOLD functional MRI at 7 Tesla. NeuroImage, 37(4):1161-1177.

Zimmermann, J., Goebel, R., De Martino, F., van de Moortele, P.-F., Feinberg, D., Adriany, G., Chaimow, D., Shmuel, A., Uğurbil, K., and Yacoub, E. (2011). Mapping the organization of axis of motion selective features in human area MT using high-field fMRI. PLoS One, 6(12):e28716. 


\section{Chapter II}

\section{Histological validation of DTI: Tractography}

Corresponding publication:

Seehaus, A. K., Roebroeck, A., Chiry, O., Kim, D.-S., Ronen, I., Bratzke, H., Goebel, R., and Galuske, R. (2013). Histological validation of DW-MRI tractography in human postmortem tissue. Cerebral Cortex, 23(2):442-450 


\section{Abstract}

Despite several previous attempts, histological validation of diffusionweighted magnetic resonance imaging (dMRI) based tractography as true axonal fiber pathways remains difficult. In the present study, we establish a method to compare histological and tractography data precisely enough for statements on the level of single tractography pathways. To this end, we used carbocyanine dyes to trace connections in human post-mortem tissue and aligned them to high resolution $\mathrm{dMRI}$ of the same tissue processed within the diffusion tensor imaging (DTI) formalism. We provide robust definitions of sensitivity (true positives) and specificity (true negatives) for DTI tractography and characterize tractography paths in terms of receiver operating characteristics. With sensitivity and specificity rates of approximately $80 \%$, we could show a clear correspondence between histological and inferred tracts. Furthermore, we investigated the effect of FA thresholds for the tractography and identified FA values between 0.02 and 0.08 as optimal in our study. Last, we validated the course of entire tractography curves to move beyond correctness determination based on pairs of single points on a tract. Thus, histological techniques, in conjunction with alignment and processing tools may serve as an important validation method of dMRI on the level of inferred tractography projections between brain areas.

\section{Introduction}

Understanding the function of the brain is not possible without precise knowledge about the connectivity between its different parts. In the last century, several methods have been developed to study the fiber connections in tissue probes, starting with simple dissection approaches and ending with highly sophisticated staining techniques. However, all of these methods require animal experiments or post mortem tissue. As a consequence, they are not suitable for questions involving structural brain connectivity in the living human. In the last two decades, magnetic 
resonance imaging has provided important new tools to address this problem. In particular, diffusion weighted imaging techniques such as diffusion tensor imaging (DTI) are capable of probing neuronal fibers in vivo. Since the introduction of DTI (Basser et al. 1994), it has been applied to a wide range of topics, especially in clinical studies. For example, the role of white matter characteristics in schizophrenia (Kanaan et al. 2005, Kubicki et al. 2005), Major Depression (Maller et al. 2010, Tham et al. 2010), Alzheimer's (Medina and Gaviria 2008, Hess 2009), and epilepsy (Richardson 2010) has been investigated using DTI. Beyond the mapping of scalar tensor measures, as applied in the above mentioned studies, DTI can also support tractography of large fiber-bundles (Conturo et al. 1999, Mori et al. 1999, Basser et al. 2000).

However, previous studies have shown that DTI has several limitations for tractography. First, it is known to perform poorly in brain areas of high fiber curvature or with fibers crossing $(X)$ or kissing $(><)$. In addition, DTI cannot resolve whether fibers are converging or diverging at Y-shaped structures because directional information cannot be provided (Roebroeck et al. 2008). Second, DTI studies are principally limited to the investigation of white matter, as the fractional anisotropy (FA), the basic information on which DTI tracking algorithms perform, is too low in gray matter. In other words, DTI needs clear diffusion profiles as given by white matter fiber bundles to perform well. Likewise, it is difficult to recognize very small fiber bundles with DTI (Conturo et al. 1999). This, however, is based on the spatial resolution of the respective scans and might be improved using more powerful MRI hardware.

Given these confounds and the uncertainty about the structural origin of the diffusion signals (Beaulieu 2002), validation methods are necessary which provide quantitative measures of how DTI performs under certain circumstances. Previous studies have addressed this issue by different methodological combinations, among them studies involving DTI phantoms (Pullens et al. 2010), MRI contrast agents (Lin et al. 2001) or anatomy atlases (Catani et al. 2002). The most commonly used approach was to compare DTI data to actual histological data, which visualized 
white matter architecture or neuronal connectivity by selective or unselective staining techniques (Kaufman et al. 2005, Dauguet et al. 2007, Leergaard et al. 2010). One of the processing steps in these studies was to reconstruct a $3 \mathrm{D}$ volume from the histological slices that is aligned to the volume obtained through the DTI measurement. This task is affected by several considerations. First, during histological processing the tissue almost inevitably gets distorted, at least by a global scaling factor in one or both of its planar directions. Second, creating a histological slice usually reduces all information from the tissue to two dimensions because the depth information within individual slices is lost. In consequence, it is difficult to identify the depth orientation of stained fiber material, which is useful for a 3D reconstruction of whole fibers. Third, histological and DTI data are substantially different: With an inherently two-dimensional high-resolution (single microns) signal on the one hand, and a 3D lowresolution (hundreds of microns) signal on the other hand, it is difficult to obtain a quantitative, valid comparison of these two data sources. So far, it has mostly been done by downsampling the histological data to the resolution of the MRI and applying a comparison sectionwise (Kaufman et al. 2005, Dauguet et al. 2007). However, in such approaches a significant amount of the information contained in the histology is lost. Most importantly, this approach precludes validating tractography algorithms using actual axonal tracts labeled from their origin or termination in the gray matter.

Therefore, the aim of our study was to implement a new approach of validating DTI-based streamline tractography on the level of single tractography pathways. To this end, we used fluorescent carbocyanine dyes to trace connections in human post-mortem tissue and compared these to DTI tractography results on the same tissue. DiI and DiA are fluorescent lipophilic tracers that are able to selectively stain fibers projecting from or to a crystal implantation location. Placing the dye in the gray matter, this property enables us to obtain information about the origin of white-matter fiber paths which is very difficult to obtain otherwise. We use this property to define true positives (sensitivity), true negatives (specificity) and result- 
ing receiver operating characteristic (ROC) curves for DTI tractogaphy in the framework of signal detection theory (SDT). After investigation of tractography performance in terms of pairs of single points on a tract, we analyze the correspondence of entire tract curves with the carbocyanine dye staining. This approach allows for the evaluation of the congruence of DTI tractography and actual structural connectivity not only for the white-matter fiber-"highways" but also for the fiber terminations that are far below the resolution of current MR hardware.

\section{Materials and Methods}

\subsection{Tissue}

The tissue probes were obtained from the brain of a single subject (male, 33 years old, no history of neurological disorders or brain injury). After a postmortem delay of 12 hours, the upper temporal lobe was dissected and immersion fixated for 4 days in a solution containing $2.6 \%$ paraformaldehyde, $0.8 \%$ iodacetic acid, $0.8 \%$ sodiumperiodate, and $0.1 \mathrm{M} \mathrm{D-L-lysine} \mathrm{in}$ $0.1 \mathrm{M}$ phosphate buffer at $\mathrm{pH}$ 7.4.

\subsection{Carbocyanine tracing}

DiI and DiA are fluorescent lipophilic tracers that progress along lipid membranes by diffusion and have for long been tested as anterograde and retrograde neuronal connection tracers (Honig and Hume 1986, Godement et al. 1987, Honig and Hume 1989). More recently, these dyes were confirmed to work also in adult human post-mortem tissue (Burkhalter et al. 1993, Galuske et al. 2000). Four carbocyanine crystals (two DiI, two DiA) were implanted into the upper surface of the block at a depth of $500-1000 \mu \mathrm{m}$. The distance between each crystal of the same dye was about $3 \mathrm{~cm}$. After 48 months, the tissue was sliced at a thickness of $70 \mu \mathrm{m}$ and stained fiber material of every 5 th slice (i.e. every $350 \mu \mathrm{m}$ ) was graphically documented using fluorescent microscopy at 37x enlargement 
factor and excitation wavelengths of $546 \mathrm{~nm}$ (DiI) and 440-490 nm (DiA), respectively. One of the implantation sites had to be excluded from further analysis because its projections were mainly outside the scope of the MR scans of the respective tissue.

\subsection{Diffusion MRI}

17 months after fixation, a dMRI scan was performed. Scanning procedures were similar to those described in Roebroeck A et al. (2008): Scanning was done using a $9.4 \mathrm{~T} / 31 \mathrm{~cm}$ MR scanner (Magnex Scientific, UK) equipped with $0.003 \mathrm{~T} / \mathrm{cm}$ gradients ( $11 \mathrm{~cm}$ ID, $300 \mu$ s rise time, Magnex Scientific) and driven by a UnityINOVA console (Varian, Walnut Creek, CA) at the Center for Magnetic Resonance Research (CMRR), University of Minnesota Medical Center. The RF coil was a home-built quadrature surface coil, composed of two $3.3 \mathrm{~cm}$ diameter partially overlapping coils. Diffusion weighted MR images were acquired at room temperature using a multishot pseudo-3D double spin-echo echo-planar imaging (SE-EPI) sequence with two phase encoding directions and four segments per pseudo-slice. The following parameters were used: $T R / T E=2000 \mathrm{~ms} / 35 \mathrm{~ms},|g|=$ $0.0017 \mathrm{~T} / \mathrm{cm}, \delta=6 \mathrm{~ms}, \Delta=15 \mathrm{~ms}\left(b=1584 \mathrm{~s} / \mathrm{mm}^{2}\right.$ for each direction). Six gradient combinations according to the direction scheme $(X, Y, 0)$, $(X, 0, Z),(0, Y, Z),(X,-Y, 0),(0, Y,-Z),(-X, 0, Z)$ and one image with minimal gradient strength on three directions was acquired as the unweighted image. The field of view $(\mathrm{FOV})$ was $(5 \times 5 \times 6.4) \mathrm{cm}^{3}$, the matrix size was $128 \times 128 \times 64$ (giving a nominal resolution of $(391 \times 391) \mu \mathrm{m}$ inplane with a slice thickness of $1 \mathrm{~mm}$ ). Shimming for field inhomogeneities was done using FASTMAP to include high order shims in an analytical manner. A set of images showing raw $\mathrm{T}_{2}$-weighted (i.e. without diffusion weighting, $\left.b_{0}\right)$, apparent diffusion coefficient and fractional anisotropy contrast is shown in Figure 1. 

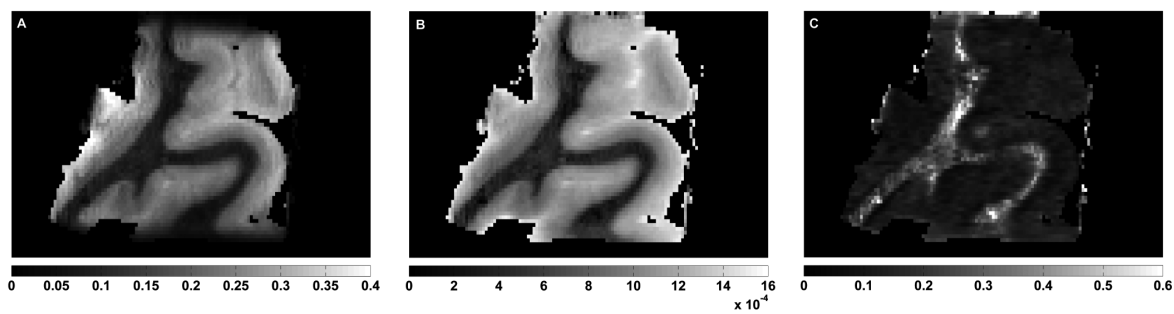

Figure 1: Typical images and calculated maps shown for one slice of the specimen used in the experiments. A: $\mathrm{T}_{2}$-weighted image $(b=0)$. The scale is given in arbitrary units. B: Apparent diffusion coefficient (ADC) map. The units are $\mathrm{mm}^{2} / \mathrm{s}$. The ADC value for white matter was between 3 and $5 \times 10^{-4} \mathrm{~mm}^{2} / \mathrm{s}$. The b-value used in the experiment $\left(b=1584 \mathrm{~s} / \mathrm{mm}^{2}\right)$ is close to optimal. C: Fractional anisotropy (FA) map. Typical values for white matter range between 0.25 and 0.5. Please note the sharp ADC differences between gray and white matter, typically not observed in in-vivo DTI measurements. Similar observations can be seen in other post-mortem dMRI studies (e.g. D'Arceuil et al. 2008).

\subsection{DTI tractography}

Diffusion-weighted MRI data were further processed and visualized in custom written software in $\mathrm{C}++$ and using OpenGL - see Roebroeck et al. (2008) for more details. Diffusion tensors were calculated for the diffusion profile of each voxel as a 3D Gaussian using a standard linear regression approach. Subsequently, tractography was performed by the Euler stepping solution for streamlines locally corresponding to the resulting tensor field (e.g. Basser et al. 2000). That is, we used a line propagation algorithm that starts from a specified seedpoint and subsequently takes steps of stepsize $\alpha$ in a direction determined by the local tensor and the previous tracking direction, terminating when the local fractional anisotropy (FA) falls below the FA threshold or the curvature (the angle between actual and previous direction) exceeds the angle threshold. The tractography parameters used were a stepsize of $100 \mu \mathrm{m}$, an angle threshold of $90^{\circ}$ and 45 seed points per source voxel. We used various FA thresholds of $0.01,0.02$, $0.04,0.06,0.08,0.10,0.15,0.20$, and 0.25 , respectively. A low FA threshold lets the algorithm draw more and longer tracts. Thus, in our approach, 
FA thresholds serve as the detection criterion for ROC analysis: a low threshold makes tractography more sensitive; a high threshold makes it more specific.

\subsection{Alignment}

In order to obtain a common reference frame for both the histology and the tractography data, first we fused the manual drawings of traces of different dyes with a set of high resolution histology photographs (Fig. 2A). The resulting histological slices were then aligned to the dMRI volume with a separate 8 parameter affine transformation ( 3 translations, 3 rotations, 2 scales) for each slice. The transformations were conducted manually by matching the gray-white matter borders as seen in the histology and a FA map (Fig. 2B) of the DTI volume. As indicated in Figures 2A and $B$, the gray-white matter boundary is a robust and useful alignment criterion in both modalities, histology and MR, that is unaffected by noise and non-matching contrast variations within tissue compartments as well as outside the tissue. Visual judgment of alignment was aided by an interactive fusion of both modalities, such that each transformation of a histological slice led to an immediate update of the FA map. In this way, each slice could be successively transformed towards an optimal fitting of the gray-white matter boundaries (Fig. 2C). Individual single slice alignment was constrained by the necessary parallelism of the histological slices, which was also used to provide an initial interpolated position for all middle slices after alignment of a few of the outer slices.

The alignment procedure resulted in a full 3D spatial coregistration of histological slices and diffusion tensors such that pixel locations in histological slices and tract coordinates in the DTI volumes could be directly compared for proximity (Fig. 2D). 


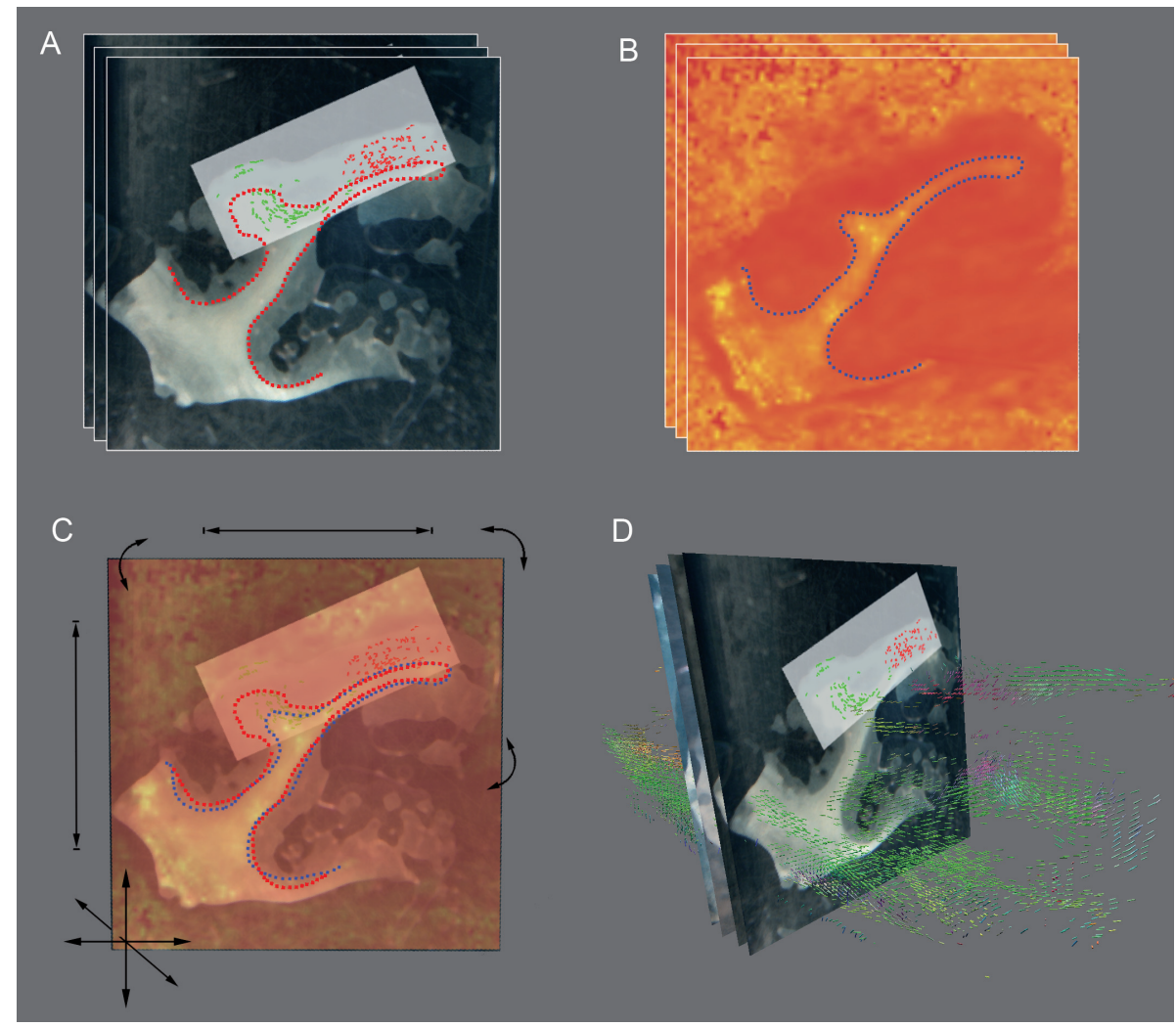

Figure 2: An illustration of the procedure and criteria for alignment of histology and MR diffusion data. A: Example of the high resolution 2D photographs of our set of co-planar histological slices. The manual drawings of traces of different dyes (in red and green) were fused with the photographs. The red dotted outline illustrates the gray-white matter boundary which was used as a criterion for alignment. B: An arbitrary 2D plane through the $3 \mathrm{D}$ fractional anisotropy map derived from the diffusion MR data, close to the optimal alignment with the histological slice in A, with its gray-white matter boundary illustrated with the blue dotted line. C: A fusion of the histology and 2D plane through the FA map which was used for alignment. Interactive and online manual changes to 3 translations, 3 rotation and 2 scale parameters (illustrated by arrows) lead to an immediate update of the FA map on the plane. These parameters were adjusted slice-by-slice to visually match the gray-white matter boundary between the two modalities and align histology to diffusion $\mathrm{MR}$ in 3D. D: Illustration of the alignment result for 4 of our histological slices rendered in the 3D space of the DTI data. Voxels with high FA values are indicated as coloured cylinders, with the colour coding for the respective directionality, in order to illustrate 3D fiber direction information. 


\subsection{Sensitivity and specificity analysis}

We evaluated the DTI tractography by answering the following two questions:

1. To what extent are real axons stained with DiI recognizable as DTI tractography pathways? (sensitivity)

2. To what extent can DTI tractography paths be trusted not to appear when there are no corresponding axons? (specificity)

To accomplish this, we conducted the following steps:

1. MR voxels within a radius of approx. $0.3-1.5 \mathrm{~cm}$ around the injection site of the dyes and with FA values of at least 0.1 were classified as either containing ("true") or lacking ("false") dye staining. To correct for possible inaccurracies of the alignment and to avoid ambiguous classifications, we defined stricter voxel classifications for the sensitivity and specificity analysis (Fig. 3A). We defined "strictly true" ("strictly false") voxels as true (false) voxels for which all 26 nearest neighbors are also classified as true (false). This procedure led to a total sample of $\mathrm{N}=13381$ source voxels, from which tractography was performed.

2. Originating from each of these voxels, we performed fiber tractography with the afore-mentioned algorithm. Subsequently, the voxels were classified as positive if any one of their fibers hit the corresponding implantation site, or negative, if not (Fig. 3B).

3. Combining step 1 and 2, voxels could now be divided into hits (true positive), misses (false negative), correct rejections (true negative), and false alarms (false positive). An illustration of the entire classification approach is given in Figure 3.

A potential confound in this approach are unequal numbers of true and false voxels or voxels which are near to or far from the implantation site (as voxels near to the injection site should be more likely to hit it, a priori). Consequently, voxel counts were balanced for these confounds using the following procedure for each of the implantations:

1. Cut the sets $T$ and $F$ of true and false voxels such that both have the 

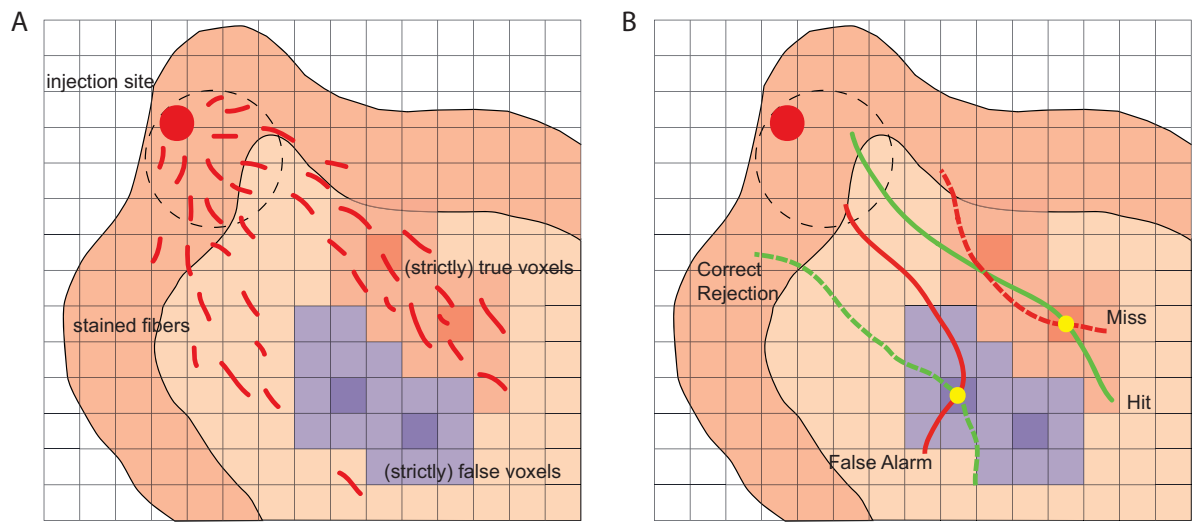

Figure 3: A: Definition of true and false voxels based on DiI staining. Strictly true (strictly false) voxels are those for which none of their 26 nearest-neighbours is a false (true) voxel. B: Definition of hit, miss, false alarm and correct rejection.

same range of distance to the injection site. This is determined by the smaller distance range found in either of the sets.

2. Divide the smaller set (let's assume: $T$ ) into three equally sized distance ranges, $T_{\text {near }}, T_{\text {medium }}$, and $T_{\text {far }}$.

3. Divide the bigger set $\mathrm{F}$ into subsets $F_{\text {near }}, F_{\text {medium }}$, and $F_{\text {far }}$ according to the same distance ranges.

4. For analyses within a certain distance range, reduce the bigger True/False-subset of the respective distance range at random to the size of the smaller subset. For overall analyses, reduce all subsets to match the size of the smallest subset.

With this procedure, we obtained the sample sizes illustrated in Table 1. In these samples, it was ensured that the subsets of true and false voxels were of equal size and had an equal distribution of distances to the injection site.

Based on these data and with the FA threshold of the tracking serving as a detection threshold parameter, we calculated the following: sensitivity ( $\frac{\text { \#hits }}{\text { \#true voxels }}$ ), specificity ( $\frac{\text { \#correct rejections }}{\text { \#false voxels }}$ ) and ROC curves (plotting sensitivity over 1-specificity for each of the 9 different FA values). As a measurement for the overall goodness of a sensitivity/specificity tradeoff, 
Table 1: Numbers of voxels analyzed

\begin{tabular}{lcccc}
\hline Implant & Overall & Near & Medium & Far \\
\hline 1 (Blue) & \multirow{2}{*}{1446} & $(3-8.3 \mathrm{~mm})$ & $(8.3-10.8 \mathrm{~mm})$ & $(10.8-12.9 \mathrm{~mm})$ \\
& & 1064 & 1064 & 482 \\
2 (Green) & 720 & $(3-6.4 \mathrm{~mm})$ & $(6.4-8.2 \mathrm{~mm})$ & $(8.2-11.9 \mathrm{~mm})$ \\
& & 240 & 978 & 978 \\
3 (Red) & \multirow{2}{*}{384} & $(3-4.5 \mathrm{~mm})$ & $(4.5-5.5 \mathrm{~mm})$ & $(5.5-9 \mathrm{~mm})$ \\
& & 128 & 230 & 308 \\
\hline
\end{tabular}

we used the Euclidean distance $D$ of its ROC-curve point to the point of perfect discrimination $(0 / 1)$. In the theoretically optimal case, where perfect discrimination is actually achieved, $D$ is 0 . The apparently worst case is $D=\sqrt{2}$ for 0 sensitivity and specificity. This value would, however, express just the same perfect discrimination, with only positive and negative responses systematically switched. The actual worst case lies on the main diagonal of the respective ROC curve, the so-called "line of no discrimination", and results in a $D$ between 1 and $\sqrt{2} / 2$.

\subsection{Entire tract analysis}

As noted above, sensitivity and specificity only depend on the connections of pairs of points on a tractography path, whereas the actual course of the entire tractography pathway is not considered. More precisely, perfect sensitivity would be concluded if every tractography path originating from a true voxel is somehow connected to the implantation site, even if by a highly unlikely path. In contrast, DTI tractography paths that are a perfect image of actual fibers should be accompanied by stained fiber material on their whole way from source voxel to implantation site. Consequently, we expected that the majority of the voxels passed by true pathways are actually true voxels (whereas the opposite should hold for false pathways). This hypothesis was statistically tested with a sample of 30 true and 30 false voxels (ten voxels for each distance range) which 
was randomly chosen within one implantation site. Originating from each of these voxels, one single fiber was tracked with the same set of FA thresholds as in the main analysis. Subsequently, the numbers of true voxels passed by these pathways were calculated and compared using unpaired t-tests.

\section{Results}

\subsection{Sensitivity/specificity}

We report detailed results from one of the three implantation sites (the "Red" site). Results from the two other sites are reported and discussed generally. The best sensitivity and specificity pairs for the fixated postmortem tissue scanned at room temperature were found for FA values between 0.02 and 0.08 . With this setting, sensitivity ranged from $70 \%$ to $78 \%$, specificity from $79 \%$ to $87 \%$. As described above, the conjoint optimality of these values was measured as the Euclidean distance $D$ from the sensitivity/specificity tradeoff achieved to the optimal value of $100 \%$ for both sensitivity and specificity. With respect to $D$, the best single value observed was $0.78 / 0.79$ for an FA threshold of 0.02 . Altogether, there is a clear discrimination for FA thresholds between 0.02 and 0.08 . There was a significant decrease for FA values of 0.1 or higher. For extremely low FA values, the loss of specificity adumbrates the increase of sensitivity, consequently resulting in a degrading discrimination as well (Table 2).

Table 3 shows the sensitivity/specificity values and optimality distance $D$ obtained for the different distance ranges. The best decision quality was achieved for near voxels, $0.82 / 0.89$ being the best single result obtained at an FA threshold of 0.08 . Similarly to the overall result, there was an optimal range of FA thresholds between 0.02 and 0.08 . Within this range, the goodness of the decision was relatively stable. Likewise, the results drop off rapidly for higher FA thresholds. 
Table 2: Overall result for one injection

\begin{tabular}{cccc}
\hline FA threshold & sensitivity & specificity & $\mathrm{D}$ \\
\hline 0.01 & 0.8229 & 0.7083 & 0.3412 \\
0.02 & 0.7813 & 0.7917 & 0.3021 \\
0.04 & 0.7656 & 0.7969 & 0.3101 \\
0.06 & 0.7292 & 0.8333 & 0.318 \\
0.08 & 0.7031 & 0.8698 & 0.3242 \\
0.1 & 0.6042 & 0.9219 & 0.4035 \\
0.15 & 0.3281 & 0.9792 & 0.6722 \\
0.2 & 0.1875 & 0.9896 & 0.8126 \\
0.25 & 0.1094 & 1 & 0.8906 \\
\hline
\end{tabular}

\subsection{ROC curves}

Figure 4 shows the ROC curves as obtained from the sensitivity/specificity values for each FA threshold. The curves start at high FA values with $100 \%$ specificity and sensitivity slightly above 0 and rise with decreasing FA to a maximum sensitivity of $70 \%$ to $90 \%$ combined with specificity values between $60 \%$ and $80 \%$. This indicates the crucial role of FA values for the manner in which the line propagation algorithm works: Low FA values lead to many long tractography paths, thus resulting in many hits (high sensitivity) but also many false alarms (low specificity). However, high values produce a more restrictive tracking with less hits (low sensitivity) but more correct rejections (high specificity). The overall goodness of an ROC curve is often assessed by the area under the curve (Fawcett 2006), which ranges between 0 and 1 . Similarly to $D$, a value of 1 means perfect discrimination and 0.5 is the theoretical worst value. To determine this value, our ROC data had to be extrapolated toward the extreme point of no specificity, which has never been reached in our experiments. Using a hypothetical extreme point of 0 specificity and $90 \%$ sensitivity, we got an area of 0.80 under the overall ROC curve, indicating a strong discrimination. 


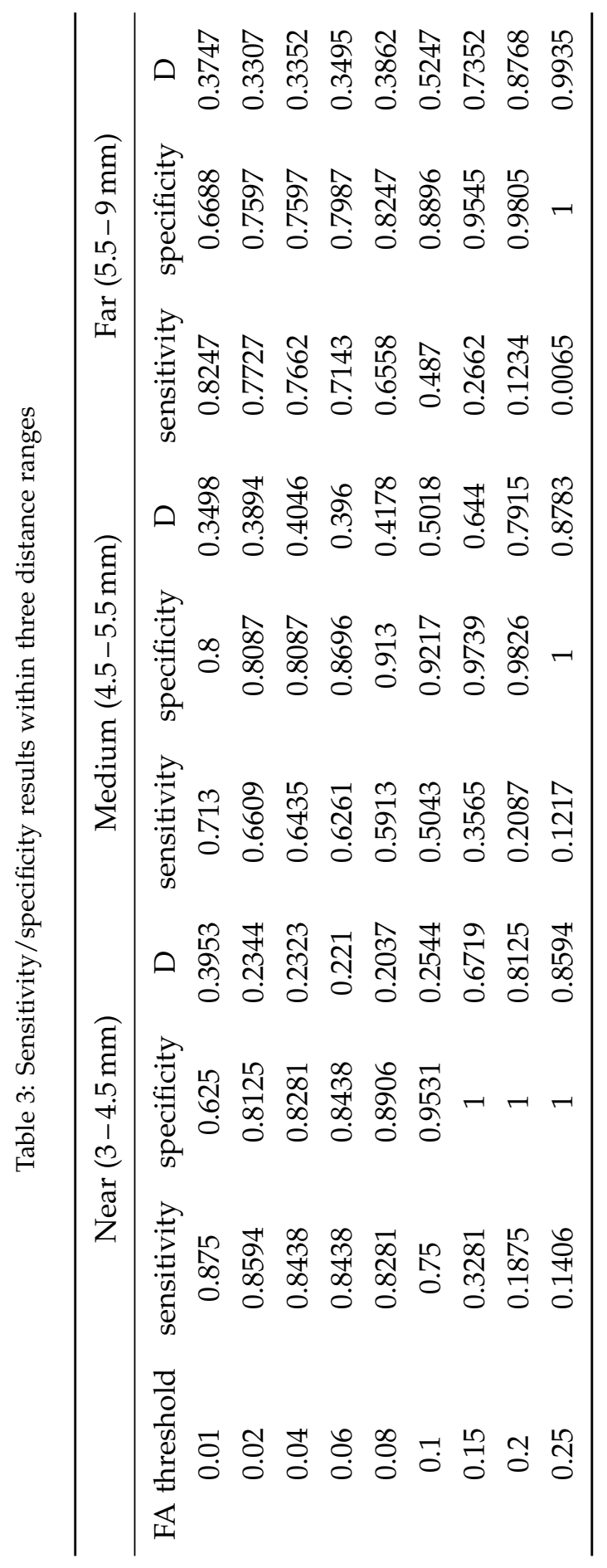



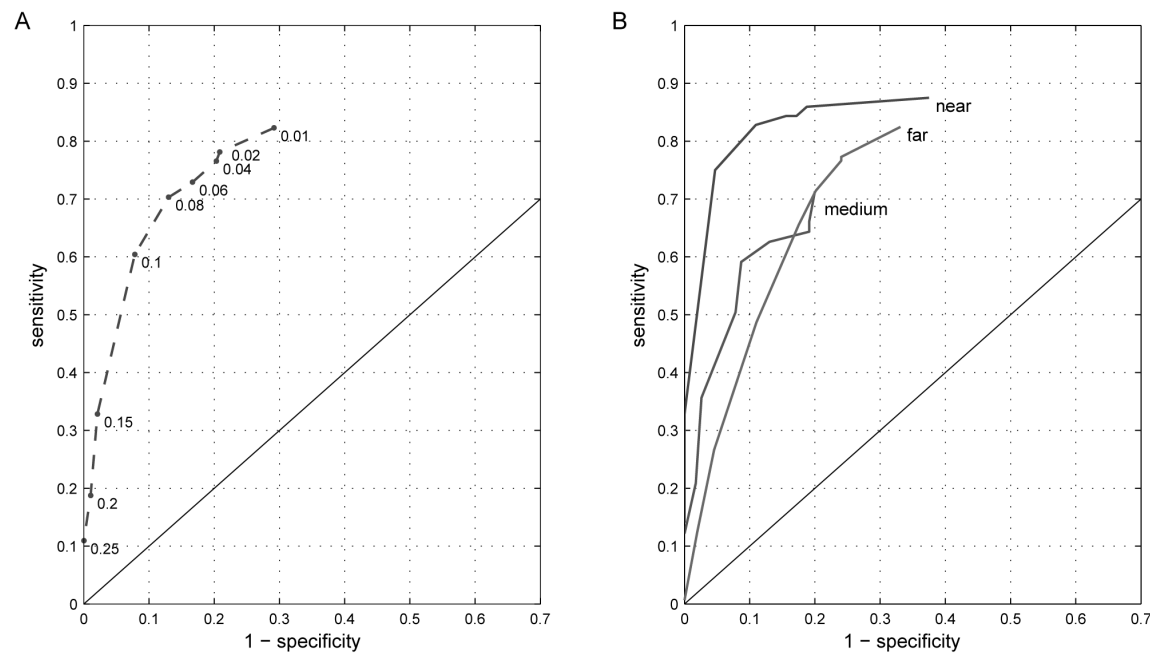

Figure 4: ROC curves obtained from a single dye implantation. A: Overall result $(n=384)$ balanced for true and false voxels over distance. The numbers next to the curve give the respective FA thresholds. B: Distinct curves for the different voxel groups: near $(3-4.5 \mathrm{~mm}$, $n=128)$, medium $(4.5-5.5 \mathrm{~mm}, n=230)$ and far $(5.5-9 \mathrm{~mm}, n=308)$ distance.

As for the different distance ranges (Fig. 4B), best general discrimination was achieved for voxels close to the implantation site ("near") with an area of 0.86 under the respective ROC curve. The more distant "medium" and "far" curves enclosed slightly smaller areas of 0.75 and 0.76 , respectively. Thus, the principal differences in discrimation behavior for different distances are in agreement with the expectation that "tractography over short distances is easier".

The remaining two implantation locations ("Blue" and "Green") showed similar specificity values of generally more than $66 \%$, yet at a specificity that was even for the lowest FA values not much higher than $60 \%$. This result was probably due to the shape of the tracing, as will be discussed below. 
Table 4: Average number of true and false voxels passed by true and false pathways (standard deviation in brackets) and the significance for an unpaired t-test for the truefalse difference.

\begin{tabular}{lccccccccc}
\hline FA threshold & 0.01 & 0.02 & 0.04 & 0.06 & 0.08 & 0.1 & 0.15 & 0.2 & 0.25 \\
\hline \multirow{2}{*}{ True path } & 17.5 & 17.5 & 17.5 & 17.5 & 16.3 & 14.2 & 9.9 & 3.5 & 1.3 \\
& $(8.8)$ & $(8.8)$ & $(8.8)$ & $(8.8)$ & $(8.1)$ & $(7.4)$ & $(7.6)$ & $(4.5)$ & $(2.4)$ \\
\multirow{2}{*}{ False path } & 3.4 & 3.4 & 3.4 & 3.3 & 2.3 & 1.9 & 0.6 & 0.067 & 0 \\
& $(4.2)$ & $(4.2)$ & $(4.2)$ & $(4.2)$ & $(3.3)$ & $(3.2)$ & $(1.2)$ & $(0.36)$ & $(0)$ \\
$p$ & $<10-7$ & $<10-7$ & $<10-7$ & $<10-7$ & $<10-8$ & $<10-7$ & $<10-6$ & $<0.001$ & $<0.01$ \\
\hline
\end{tabular}

\subsection{Entire tract analysis}

Fiber tracts originating from true voxels pass significantly more true voxels than pathways tracked from false voxels. This result could be obtained for all FA values used, but it was most pronounced for the lower half of the FA spectrum. Table 4 shows the average numbers of true voxels passed by true and false pathways for each FA value. The most significant result was obtained for an FA threshold of 0.08. For this value, Figure 5 shows the distribution of true and false voxels passed for both true and false fibers as defined above. It is obvious that all true pathways contain many true voxels on their way (Fig. 6A), while most of the false pathways contain very few or no true voxels, with a few outliers (Fig. 6B). FA thresholds of 0.04 and lower yield the same result in this analysis, as the respective pathways have reached their maximal length within the area of the staining. In addition, these pathways tend to reach far beyond this area and are mostly too long and too curved as to represent real fiber bundles (Fig. 6C).

\section{Discussion}

\subsection{DTI tractography}

We report sensitivity/specificity values of up to $0.78 / 0.79$ over all tractography and an area under the ROC curve of 0.80 . This is a convincing 


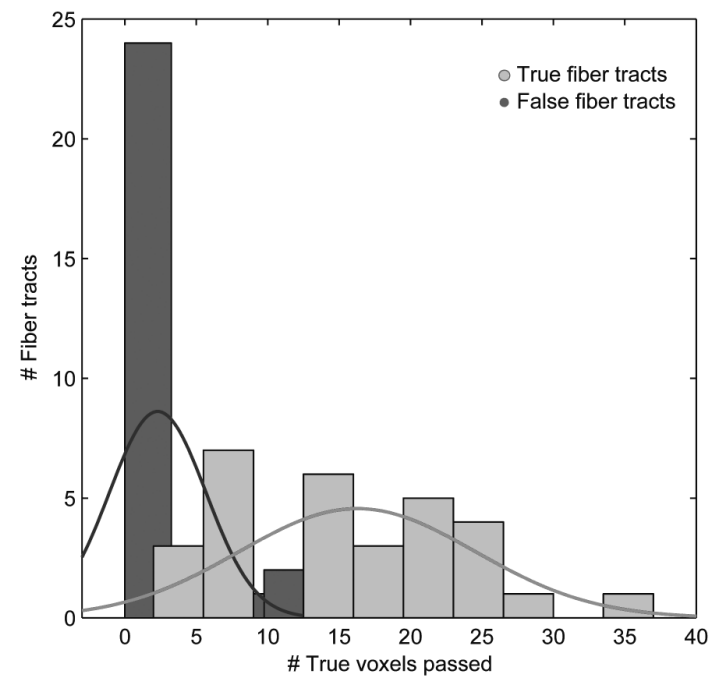

Figure 5: Histogram of numbers of true voxels passed by true tractograpy pathways (light gray) and false tractograpy pathways (dark gray). Many more true voxels are passed by true pathways than by false pathways.

accuracy, especially as compared to previous ROC analyses of DTI measurements (Iturria-Medina et al. 2011).

This result has been obtained in full only for one of the three injections, in the other two, we detected a high specificity but a low sensitivity. This observation is most likely due to the different pathways of fiber tracts arising from the different injections (Fig. 7). While in the case which offered both, high sensitivity and specificity, a discrete bundle of fibers was stained in the white matter, the other two injections provided longer (up to 13 instead of $9 \mathrm{~mm}$ ) and more diffuse projections which were directed in several directions.

Thus, the low sensitivity in these cases may reflect the weakness of DTI to track pathways through areas with many crossing pathways. Moreover, this shows a methodological limitation in the validation approach because there may be many voxels that count as true voxels due to just a few single axonal structures, which in fact do not represent DTI tractography 


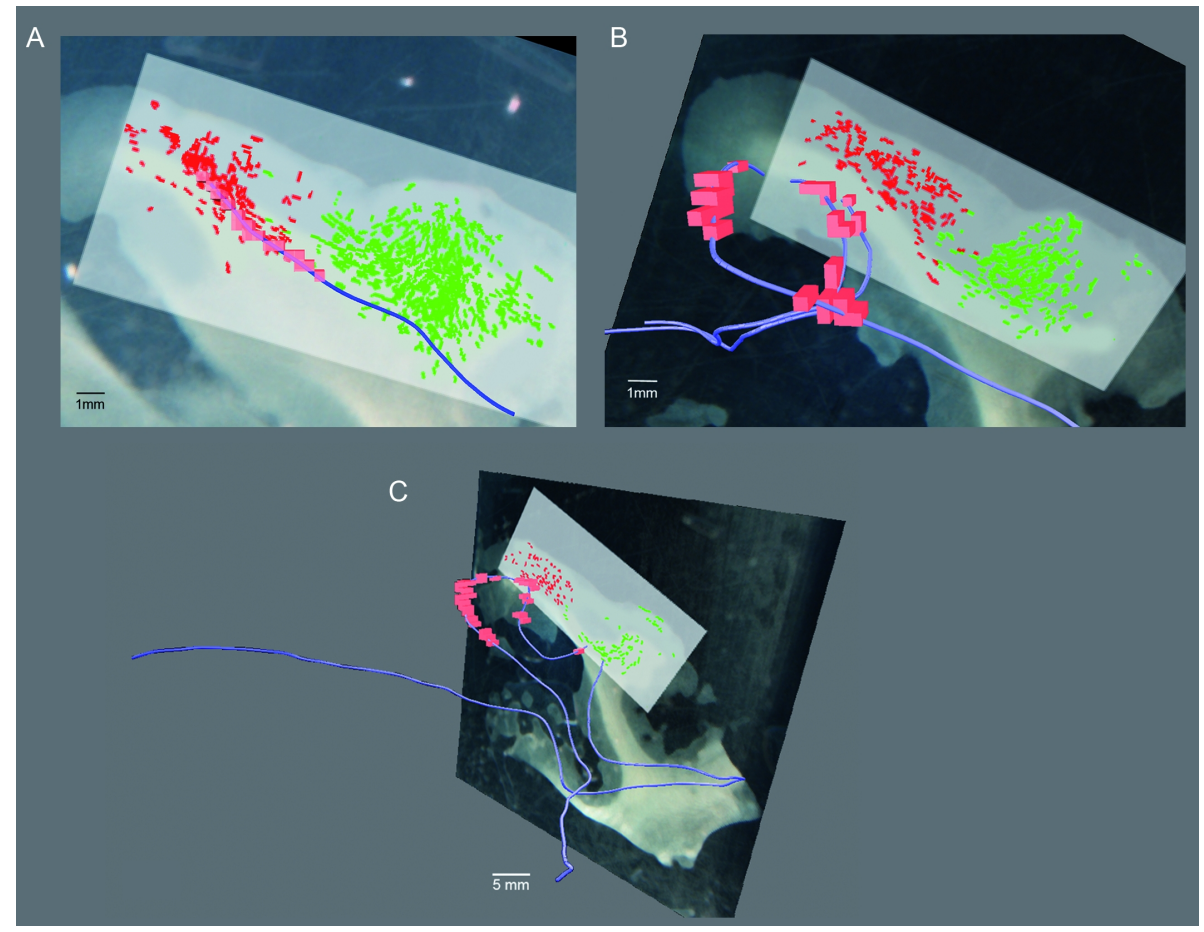

Figure 6: A: True positive dMRI tractography pathway passing through many true voxels (transparent red). Red and green: different fluorescent crystal tracings. B: False fibers that nevertheless contain many true voxels. C: True fiber coursing through many true voxels but having unrealistically long and tangled shape.

pathways. Therefore, these true voxels lead to a high number of misses and, thus, strongly interfere with the sensitivity. Consequently, this validation method currently works most accurately and unambiguously when areas are chosen where staining provides unambiguous directions of the projections. Note that this is also exactly the case in which DTI based tractography is expected to work a priori: single fiber bundles without complex fiber architecture, such as strong intra-voxel curvature or fiber crossing. Thus the current validation approach is able to robustly quantify the sensitivity and specificity of DTI based tractography on an entire pathway level away from problematic complex fiber architecture regions. One might even reverse the argument and infer complex fiber architectures from low sensitivity/specificity patterns. Therefore, a detailed analysis 


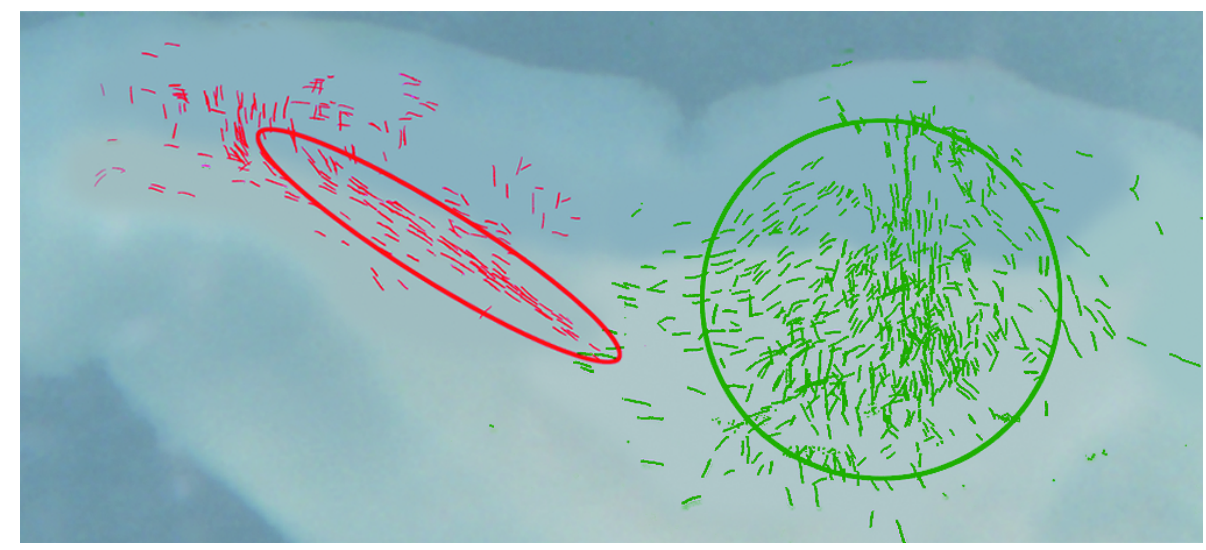

Figure 7: Projections of two injections, the left one with a discrete pathway, the right one with several staining directions.

of error sources in this validation approach is needed in order to obtain a better view of the effect of fiber architecture on sensitivity and specificity.

Another very interesting perspective for future research would be to extend this validation approach to validate tractography based on intra-voxel diffusion models that can represent complex white matter architecture, such as Diffusion Spectrum Imaging (Wedeen et al. 2008) and Constrained Spherical Deconvolution (Tournier et al. 2007). In its most advanced form, this would require delineation from histology of individual or very small bundles of axons along larger parts of their path through white matter. It is an important open question whether the type of histological processing that will best support this is a form of carbocyanine dye diffusion, as used here, a more classical axon stain photographed at high resolution, or a technique that is sensitive to the local direction of axons in white matter at very high resolution, such as Polarized Light Imaging (Axer et al. 2011). Irrespective of which approach may turn out to be the best, the sensitivity and specificity definition and approach to ROC curve analysis for entire tractography pathways as demonstrated in the present study can be transferred to other validation approaches.

Alignment of histology and diffusion MRI data is an important concern in any histological validation effort. In the present study, a manual slice-by- 
slice adjustment was used which was based on a match of the white-gray matter boundaries between the two modalities. Although tedious, this process led to a precise alignment which was undisturbed by irrelevant image features. For future efforts it is desirable to perform automatic alignment of histological slices and MR images, in particular when dealing with a higher amount of data. This could for instance be guided by a mutual information criterion and might be complemented with edgedetecting image filtering techniques. Nevertheless, recent work shows that manual intervention is often still required with automated procedures (Choe et al. 2011). In principle, such procedures can also allow for more complex non-global or non-linear alignment transformations. However, the application of these techniques to the specific alignment problem here requires considerable efforts in fine-tuning the respective parameters. These include immunity to the large differences in spatial resolution and elementary contrast in the two modalities and focusing on important image regions (such as tissue boundaries) while ignoring irrelevant ones.

Signal detection theory (SDT) has been an important and widely used validation tool in medical and psychophysical fields for several decades (for review, see Zou 2002) and perfectly suits our approach. The main advantage of SDT for our studies is that it does not compute a direct comparison of two data sets but rather evaluates whether their outcome is the same. Therefore, it allows to compare data as different as a DTI volume and a series of histological slices. Particularly, a SDT analysis is relatively independent of the resolution and does not lose the microscopic information obtained from histology. Moreover, it is possible to determine sensitivity and specificity in their full dependence on the free parameters of the tractography algorithm, e.g. FA or curvature thresholds. The resulting receiver operating characteristic (ROC) curves give insight into the effect of those parameters on DTI tractography and can effectively be used as tuning curves. Thus, this approach does not only provide a statement about the overall goodness of inferred DTI tracts but also helps to adjust tractography algorithms optimally. This has been exemplified 
by optimizing the FA threshold of the line propagation algorithm in our study.

The ROC curves achieved here have typical shapes and clearly show the role of the FA threshold as the main detection threshold parameter of the line propagation algorithm. That is, pathways can be tracked more conservatively for high or more permissively for low FA thresholds. The optimal sensitivity/specificity tradeoff was obtained for FA values between 0.02 and 0.08 . However, values greater than 0.1 should not be chosen because the tracking algorithm is likely to be overly restrictive under these conditions and consequently performs poorly. We evaluated the plausibility of these findings by examination of the whole pathway of selected tractography. This analysis revealed an optimal FA setting at 0.08 for two reasons. First, at this value true pathways showed large amounts of true (that is: stained) voxels on their pathway. Second, these pathways were biologically plausible, as opposed to pathways tracked with lower FA thresholds. Combining both results, FA thresholds of around 0.08 are likely to yield the best concordance of inferred and real fiber paths. This finding is in good accordance with a previous study which identified FA values around 0.10 as optimal (Dauguet et al. 2007).

It is important to note that the effect of FA threshold on tractography results and its optimal value is obtained for paraformaldehyde-fixated post-mortem tissue, scanned at room temperature (rather than body temperature). Although water diffusion directional anisotropy has been shown to be preserved in fixated tissue (Guilfoyle et al. 2003, Sun et al. 2003, 2005), both the chemical fixation process and the lower temperature is expected to lower the absolute value of apparent diffusion coefficients (ADC) and FA. Thus, the FA thresholds for tractography in this tissue are diffcult to be transferred to in vivo measurements.

\subsection{Evaluation method}

There have been several approaches to evaluate diffusion based imaging techniques with histological methods. In the majority of these studies, 3D 
images were reconstructed from a series of histological slices and consequently the overlap of DTI and the histological volumes was evaluated (e.g. Dauguet et al. 2007). Approaches of this kind bear some inherent limitations. Most notably, they are very vulnerable to inaccuracies in the registration process, and therefore require either high tolerance thresholds or very simply structured tissue probes to obtain acceptable results. An alternative approach which is more robust against distortions, is the receiver operator approach applied here which has been successfully used in recent studies (Iturria-Medina et al. 2011). Moreover, an ROC curve has the additional advantage over Pearson correlation based approaches that it provides insight into the dynamic effect of any experimental parameter. That is, rather than just detecting the optimal setting of the parameters, it can precisely reveal the effect of the parameters in terms of sensitivity and specificity.

\subsection{Conclusion}

In summary, we can conclude that a) DTI can principally reflect the shape and orientation of nerve pathways even on a small spatial scale, and b) that using our approach of diffusive dye staining that selectively marks entire single axons and ROC curve analysis, it is feasible to validate DTI on this level. Medium FA values of about 0.08 seem to be the best choice for DTI-based tractography on fixed post-mortem tissue at room temperature when considering ROC curve analysis and tractography pathway analysis together. Given our results of approximately $80 \%$ sensitivity and specificity, we have been able to clearly demonstrate that this evaluation method reflects the concordance of DTI and histological measurements in noncomplex fiber architecture regions and is suitable for further evaluation studies of diffusion weighted MR imaging and tractography approaches that may address issues such as crossing and sharp intra-voxel curvature. 


\section{References}

Axer, M., Amunts, K., Grässel, D., Palm, C., Dammers, J., Axer, H., Pietrzyk, U., and Zilles, K. (2011). A novel approach to the human connectome: Ultra-high resolution mapping of fiber tracts in the brain. NeuroImage, 54:1091-1101.

Basser, P., Mattiello, J., and LeBihan, D. (1994). Estimation of the effective self-diffusion tensor from the NMR spin echo. Journal of Magnetic Resonance, Series B, 103:247-254.

Basser, P., Pajevic, S., Pierpaoli, C., Duda, J., and Aldroubi, A. (2000). In vivo fiber tractography using DT-MRI data. Magnetic Resonance in Medicine, 44:625-632.

Beaulieu, C. (2002). The basis of anisotropic water diffusion in the nervous system - a technical review. NMR in Biomedicine, 15:435-455.

Burkhalter, A., Bernardo, K., and Charles, V. (1993). Development of local circuits in human visual cortex. Journal of Neuroscience, 13:1916-1931.

Catani, M., Howard, R., Pajevic, S., and Jones, D. (2002). Virtual in vivo interactive dissection of white matter fasciculi in the human brain. NeuroImage, 17:77-94.

Choe, A. S., Gao, Y., Li, X., Compton, K. B., Stepniewska, I., and Anderson, A. (2011). Accuracy of image registration between MRI and light microscopy in the ex vivo brain. Magnetic Resonance Imaging, 29:683692.

Conturo, T., Lori, N., Cull, T., Akbudak, E., Snyder, A., Shimony, J., McKinstry, R., Burton, H., and Raichle, M. (1999). Tracking neuronal fiber pathways in the living human brain. Proceedings of the National Academy of Sciences of the United States of America, 96:10422-10427.

D'Arceuil, H., Liu, C., Levitt, P., Thompson, B., Kosofsky, B., and De Crespigny, A. (2008). Three-dimensional high-resolution diffusion 
tensor imaging and tractography of the developing rabbit brain. Developmental neuroscience, 30(4):262-275.

Dauguet, J., Peled, S., Berezovskii, V., Delzescaux, T., Warfield, S., Born, R., and Westin, C. (2007). Comparison of fiber tracts derived from in-vivo dti tractography with 3D histological neural tract tracer reconstruction on a macaque brain. NeuroImage, 37:530-538.

Fawcett, T. (2006). An introduction to ROC analysis. Pattern Recognition Letters, 27:861-874.

Galuske, R., Schlote, W., Bratzke, H., and Singer, W. (2000). Interhemispheric asymmetries of the modular structure in human temporal cortex. Science, 289:1946-1949.

Godement, P., Vanselow, J., Thanos, S., and Bonhoeffer, F. (1987). A study in developing visual systems with a new method of staining neurones and their processes in fixed tissue. Development, 101:697-713.

Guilfoyle, D., Helpern, J., and Lim, K. (2003). Diffusion tensor imaging in fixed brain tissue at 7.0 T. NMR in Biomedicine, 16:77-81.

Hess, C. (2009). Update on diffusion tensor imaging in Alzheimer's disease. Magnetic Resonance Imaging Clinics of North America, 17:215-224.

Honig, M. G. and Hume, R. I. (1986). Fluorescent carbocyanine dyes allow living neurons of identified origin to be studied in long-term cultures. Journal of cell biology, 103:171 - 87.

Honig, M. G. and Hume, R. I. (1989). Dil and diO: versatile fluorescent dyes for neuronal labelling and pathway tracing. Trends in neurosciences, 12:333-341.

Iturria-Medina, Y., Perez Fernandez, A., Morris, D., Canales-Rodriguez, E., Haroon, H., Penton, L., Augath, M., Galan, L., Logothetis, N., Parker, G., and Melie-Garcia, L. (2011). Brain hemispheric structural efficiency and interconnectivity rightward asymmetry in human and nonhuman primates. Cerebral Cortex, 21:56-67. 
Kanaan, R., Kim, J., Kaufmann, W., Pearlson, G., Barker, G., and McGuire, P. (2005). Diffusion tensor imaging in schizophrenia. Biological Psychiatry, 58:921-929.

Kaufman, J., Ahrens, E., Laidlaw, D., Zhang, S., and Allman, J. (2005). Anatomical analysis of an aye-aye brain (Daubentonia madagascariensis, primates: Prosimii) combining histology, structural magnetic resonance imaging, and diffusion-tensor imaging. The Anatomical Record, Part A: Discoveries in Molecular, Cellular, and Evolutionary Biology, 287:1026-1037.

Kubicki, M., Westin, C., McCarley, R., and Shenton, M. (2005). The application of DTI to investigate white matter abnormalities in schizophrenia. Annals of the New York Academy of Sciences, 1064:134-148.

Leergaard, T., White, N., de Crespigny, A., Bolstad, I., D'Arceuil, H., Bjaalie, J., and Dale, A. (2010). Quantitative histological validation of diffusion MRI fiber orientation distributions in the rat brain. PLoS One, 5:e8595.

Lin, C., Tseng, W., Cheng, H., and Chen, J. (2001). Validation of diffusion tensor magnetic resonance axonal fiber imaging with registered manganese-enhanced optic tracts. NeuroImage, 14:1035-1047.

Maller, J., Thompson, R., Lewis, P., Rose, S., Pannek, K., and Fitzgerald, P. (2010). Traumatic brain injury, major depression, and diffusion tensor imaging: Making connections. Brain Research Reviews, 64:213-240.

Medina, D. and Gaviria, M. (2008). Diffusion tensor imaging investigations in Alzheimer's disease: the resurgence of white matter compromise in the cortical dysfunction of the aging brain. Neuropsychiatric Disease and Treatment, 4:737-742.

Mori, S., Crain, B., Chacko, V., and van Zijl, P. (1999). Three-dimensional tracking of axonal projections in the brain by magnetic resonance imaging. Annals of Neurology, 45:265-269.

Pullens, P., Roebroeck, A., and Goebel, R. (2010). Ground truth hardware 
phantoms for validation of diffusion-weighted MRI applications. Journal of Magnetic Resonance Imaging, 32:482-488.

Richardson, M. (2010). Current themes in neuroimaging of epilepsy: Brain networks, dynamic phenomena, and clinical relevance. Clinical Neurophysiology, 121:1153-1175.

Roebroeck, A., Galuske, R., Formisano, E., Chiry, O., Bratzke, H., Ronen, I., Kim, D., and Goebel, R. (2008). High-resolution diffusion tensor imaging and tractography of the human optic chiasm at 9.4 T. NeuroImage, 39:157168.

Seehaus, A. K., Roebroeck, A., Chiry, O., Kim, D.-S., Ronen, I., Bratzke, H., Goebel, R., and Galuske, R. (2013). Histological validation of DW-MRI tractography in human postmortem tissue. Cerebral Cortex, 23(2):442450 .

Sun, S., Neil, J., Liang, H., He, Y., Schmidt, R., Hsu, C., and Song, S. (2005). Formalin fixation alters water diffusion coefficient magnitude but not anisotropy in infarcted brain. Magnetic Resonance in Medicine, 53:1447-1451.

Sun, S., Neil, J., and Song, S. (2003). Relative indices of water diffusion anisotropy are equivalent in live and formalin-fixed mouse brains. Magnetic Resonance in Medicine, 50:743-748.

Tham, M., Woon, P., Sum, M., Lee, T., and Sim, K. (2010). White matter abnormalities in major depression: Evidence from post-mortem, neuroimaging and genetic studies. Journal of Affective Disorders, 132:26-36.

Tournier, J.-D., Calamante, F., and Connelly, A. (2007). Robust determination of the fibre orientation distribution in diffusion MRI: Nonnegativity constrained super-resolved spherical deconvolution. NeuroImage, 35(4):1459-1472.

Wedeen, V., Wang, R., Schmahmann, J., Benner, T., Tseng, W., Dai, G., Pandya, D., Hagmann, P., D'Arceuil, H., and de Crespigny, A. (2008). 
Diffusion spectrum magnetic resonance imaging (DSI) tractography of crossing fibers. NeuroImage, 41(4):1267-1277.

Zou, K. (2002). Receiver operating characteristic (ROC) literature research. http:/ /www.spl.harvard.edu/archive/spl-pre2007/pages/ppl/ zou/roc.html. [Online bibliography; accessed 08/15/2011]. 


\section{Chapter III}

\section{Histological validation of DTI: Diffusion Tensors}

Corresponding publication:

Seehaus, A., Roebroeck, A., Bastiani, M., Fonseca, L., Bratzke, H., Lori, N., Vilanova, A., Goebel, R., and Galuske, R. (2015). Histological validation of high-resolution DTI in human post mortem tissue. Frontiers in neuroanatomy, 9 


\section{Abstract}

Diffusion tensor imaging (DTI) is amongst the simplest mathematical models available for diffusion magnetic resonance imaging, yet still by far the most used one. Despite the success of DTI as an imaging tool for white matter fibers, its anatomical underpinnings on a microstructural basis remain unclear. In this study, we used 65 myelin-stained sections of human premotor cortex to validate modeled fiber orientations and oft used microstructure-sensitive scalar measures of DTI on the level of individual voxels. We performed this validation on high spatial resolution diffusion MRI acquisitions investigating both white and gray matter. We found a very good agreement between DTI and myelin orientations with the majority of voxels showing angular differences less than 10 degrees. The agreement was strongest in white matter, particularly in unidirectional fiber pathways. In gray matter, the agreement was good in the deeper layers highlighting radial fiber directions even at lower fractional anisotropy (FA) compared to white matter. This result has potentially important implications for tractography algorithms applied to high resolution diffusion MRI data if the aim is to move across the gray-white matter boundary. We found strong relationships between myelin microstructure and DTI-based microstructure-sensitive measures. High FA values were linked to high myelin density and a sharply tuned histological orientation profile. Conversely, high values of mean diffusivity (MD) were linked to bimodal or diffuse orientation distributions and low myelin density. At high spatial resolution, DTI-based measures can be highly sensitive to white and gray matter microstructure despite being relatively unspecific to concrete microarchitectural aspects.

\section{Introduction}

Diffusion Magnetic Resonance Imaging (dMRI) is a widely used MRI technique in clinical as well as basic neuroscience applications to reveal neuronal fiber structures non-invasively in the living brain. A dMRI ac- 
quisition provides information about water diffusion in several directions in space. Usually this information is integrated in a mathematical model to give a unified description of the diffusion in one voxel.

One of the mathematically simplest and yet the most widely used of these models is diffusion tensor imaging (DTI) (Basser et al. 1994; see Mori and Zhang 2006 for an overview). In a diffusion tensor, diffusion is characterized as a Gaussian function with 3 orthogonal diffusion axes along with their characteristic lengths. Different characteristics of diffusion tensors are described by scalar measures such as mean diffusivity (MD; the average axis length) or fractional anisotropy (FA; the normalized variance of diffusion over the axes). MD, in particular, has been successfully used in a range of clinical diagnostic cases (Sundgren et al. 2004). FA is widely used in neuroscience and pre-clinical investigations as a sensitive marker of white matter microstructure (Kanaan et al. 2005, Medina and Gaviria 2008, Richardson 2010), but its microstructural basis is still under debate (Assaf et al. 2013, Assaf and Basser 2005, Jones et al. 2013). Furthermore, DTI can be used for tractography, which is the algorithmic concatenation of neighboring tensors, yielding inferred fiber pathways. The latter is an important tool in in-vivo brain connectivity research (Catani and Thiebaut de Schotten 2008, Mori et al. 1999), including the relatively young field of human connectomics (Hagmann et al. 2008, Sporns et al. 2005).

Despite its extensive usage, DTI has the drawback in tractography of being a rather unspecific unidirectional model. Its orientation estimation works very well in areas characterized by prominent fiber pathways following one direction, giving rise to a unimodal water diffusivity profile. This may often be the case, especially in white matter. However, when several different diffusion directions are present in one voxel, the directionality information of the estimated diffusion tensor is imprecise at best or even systematically biased (Jones 2010, Wedeen et al. 2008). This is particularly the case in gray matter where neurites more frequently run in at least two orthogonal or non-orthogonal directions. But also in white matter this is a known problem, recently estimated to occur in up to $90 \%$ of all voxels 
(Jeurissen et al. 2013), when fiber tracts cross or touch each other, converge or diverge (Roebroeck et al. 2008).

In order to approach these problems and to gain a better understanding of the exact nature of the dMRI signal and its modeling on the anatomical level, it is very important to conduct validation studies, which compare dMRI data to other sources of information. This information can for example come from phantoms (Pullens et al. 2010), MRI contrast agents (Lin et al. 2001), anatomical atlases (Catani et al. 2002), or a direct comparison of dMRI findings to the actual histological situation in post-mortem tissue (Leergaard et al. 2010, Seehaus et al. 2013). The latter approach is particularly promising for two reasons: First, it allows for a direct comparison of findings gained in both modalities in the very same tissue. Second, it allows for the comparison of different anatomical structures to the dMRI-findings since histological stains can be varied in order to visualize different architectural aspects, such as cell-bodies, dendrites, or myelin.

In two recent studies, Budde and colleagues propose a methodological approach for the validation of DTI with histology (Budde and Frank 2012, Budde and Annese 2013). Using structure tensors to detect local image orientation, they extracted microanatomical orientations from digitized stained tissue sections. Pooling this information over image compartments that correspond to the voxel size in a hypothetical imaging experiment, they derived fiber orientation distributions (FODs) which structurally closely resemble real dMRI data. They illustrate their method on a range of different stains in rat and human brain. They also report promising results of a preliminary comparison to actual DTI data where histological anisotropy values were averaged over selected ROIs in the rat brain and correlated with FA averages of the same ROIs.

In our study, we apply this analytical approach to a series of 65 myelinstained human brain sections in order to validate the orientational and microstructure information obtained from DTI data acquired on the same tissue probe. We extend previous studies by providing for the first time 
a direct voxel-based comparison of DTI and myeloarchitecture over a relatively large volume of human tissue focusing both on white matter and gray matter. In doing so, our main research questions were: First, how do the orientations of diffusion tensors match those of the underlying fiber material in the presence of both unidirectional and crossing pathways? This question is particularly important in the light of DTI tractography where fiber pathways are modeled by concatenating diffusion tensors along their primary orientations. Second, how do the most important scalar indices of diffusion tensors, FA, MD, and radial diffusivity (RD), relate to myeloarchitecture? Third, how does the microstructural environment, and its quantification by dMRI, change in gray matter? So far, diffusion imaging has mostly been used in white matter, however, there is an increasing number of recent studies investigating human gray matter with high resolution dMRI (Kleinnijenhuis et al. 2013, Leuze et al. 2014).

\section{Materials and Methods}

\subsection{Tissue acquisition}

This study was performed on a block of cortical tissue $(38.9 \times 36.3 \times$ $23.8 \mathrm{~mm}$ ) which comprised parts of primary motor and medial and lateral premotor cortex. The tissue was obtained 6 hours post mortem from the left hemisphere of a female subject, aged 38, without known neurological or psychiatric disorders. All procedures were approved by the ethical committee of the University Hospital Frankfurt/M. The tissue was prepared and fixed for 48 hours using a solution containing $2.6 \%$ paraformaldehyde, $0.8 \%$ iodoacetic acid, $0.8 \%$ sodium periodate, and $0.1 \mathrm{M}$ D-L-lysine in $0.1 \mathrm{M}$ phosphate buffer at $\mathrm{pH} 7.4$ at $4^{\circ} \mathrm{C}$. For long term storage, it was then transferred to a $2 \%$ paraformaldehyde solution in $0.1 \mathrm{M}$ phosphate buffer at $\mathrm{pH}$ 7.4. MR scans were performed after about 1 year of fixation. The tissue was scanned in a cylindrical container immersed in the paraformaldehyde solution in order to assure long-term preservation for later histological processing. 


\subsection{Diffusion MRI}

Measurements were performed on a small-bore $9.4 \mathrm{~T}$ system equipped with a $12 \mathrm{~cm} \mathrm{ID,} 600 \mathrm{mT} / \mathrm{m}, 100 \mu$ s rise time gradient coil and interfaced to a Siemens console. A $7 \mathrm{~cm}$ loop-coil was used for RF transmission and signal reception. A 2D spin-echo sequence was modified to include a diffusion preparation module. The measurement parameters included: FOV $(54 \times 54) \mathrm{mm}$, matrix $160 \times 160,97$ contiguous $340 \mu \mathrm{m}$ slices (achieving isotropic resolution of $340 \mu \mathrm{m}), T R=10000 \mathrm{~ms}, T E=45 \mathrm{~ms}, \Delta=22.5 \mathrm{~ms}$, $\delta=3 \mathrm{~ms}$, flip angle $=90^{\circ}, 4$ averages, $b=3000 \mathrm{~s} / \mathrm{mm}^{2}, 60$ diffusion encoding directions (obtained by an electrostatic repulsion algorithm on the whole sphere) and six $b=0$ acquisitions. The temperature in the scanner was raised to $30^{\circ} \mathrm{C}$ using an in-bore animal warming system and constantly monitored with a temperature probe.

The signal-to-noise ratio of the acquired data was estimated in the $b=0$ acquisitions as the signal within the tissue divided by the standard deviation in an image corner free of signal and evaluated to 51. Diffusion data were preprocessed in order to correct for image shift and geometric distortions arising from eddy currents induced by diffusion gradients using the FMRIB's Diffusion Toolbox available in FSL (Jenkinson et al. 2012). The estimated transformation matrices were used to transform the diffusion gradient directions accordingly (Leemans and Jones 2009). Manual segmentation of the averaged non-diffusion-weighted (i.e. pure $\mathrm{T}_{2}$-weighted) volumes was performed to obtain white and gray matter masks. Diffusion tensors (DTs) were fitted to the acquired data by linear regression using a least square minimization approach. For the diffusion tensors, FA, MD, and RD were determined.

\subsection{Histological processing}

After the MR scan, the block was cut into two halves, with the cutting plane approximating the xy-plane of the MR space. This was possible due to orientation marks on the container the tissue was scanned in, as 
well as photographic documentation of the tissue positioning within that container. The anterior part was sectioned at a slice thickness of $60 \mu \mathrm{m}$ using a microtome (Reichert-Jung, Supercut 2050) with a freezing stage (Leica, Frigomobil). To facilitate orientation within the tissue material later on, blockface photos were taken of every second section (Choe et al. 2011). This resulted in 343 coronal sections, from which every 5th one was stained for myelin using the Gallyas method (Gallyas 1979, see supplementary material for a detailed staining protocol). In this manner, we obtained 69 stained sections. Of those, 4 had to be discarded due to damage during the staining procedure, leaving 65 sections for microscopical analysis.

Furthermore, every 20th section was Nissl-stained for the identification of cortical layers and area classification. In accordance with the atlas of Economo and Koskinas (1925), the analysis of the Nissl-stained sections confirmed our macroscopic area classification during tissue acquisition. Myelin stained sections were digitized using a microscope system consisting of microscope (Zeiss, AxioImager Z1), motorized stage (Märzhäuser), and camera (Zeiss, Axiocam HRm). Digital images were obtained with a $5 x$ magnification objective as series of $1388 \times 1040$ px sized tiles, which were automatically merged using the built-in stitching algorithm (Zeiss, MosaiX). The resulting images were 8 bit grayscale, with a pixel resolution of $1.3 \mu \mathrm{m}^{2}$.

\subsection{Histological orientation analysis}

The aim of the histological analysis was to obtain fiber orientation distributions from image compartments equaling the DTI voxel size. This was achieved in a stepwise process:

\section{Image preprocessing}

Histological procedures such as the one used here usually stress the tissue both physically and chemically, resulting in tissue damage such as tears and ripples. Particularly around vessels, the tissue sections develop holes 
during the process of exsiccation. As a first step, these damaged parts had to be discarded, which was done by means of manual segmentation, using standard image processing software (Adobe Photoshop CS2). Additionally, we discarded parts of the tissue which did not contain stained fibers, mostly situated in cortical layers I and II.

\section{Structure tensor calculation}

There are several different approaches to calculate orientedness in digital images, including integral transformations like Fourier or Wavelet decomposition (Kemao and Asundi 2002, Lefebvre et al. 2011), oriented filters (Michelet et al. 2007), or template matching (Bartsch et al. 2012). Because of its recent success, we chose the structure tensor approach (Bigün and Granlund 1987), which is based on local gray level gradients. The following algorithm was conducted on each histological section image: First, the image gradient $\left(f_{x}, f_{y}\right)$ was calculated by convolution of the image matrix with the partial derivatives $G_{x}, G_{y}$ of a rotationally symmetric Gaussian kernel $G$ (size 9 pixels, $\sigma=2$ ).

From these gradient images, the structure tensors (STs) were acquired as

$$
J=\left[\begin{array}{ll}
f_{x x} & f_{x y} \\
f_{x y} & f_{y y}
\end{array}\right]
$$

where $f_{x x}$ is the pointwise product of $f_{x}$ with itself, smoothed by convolution with $G$ (analogously for $f_{x y}, f_{y y}$ ). Subsequently, in each pixel the eigenvalues $\lambda_{1}, \lambda_{2}$ (where we will assume $\lambda_{1} \geq \lambda_{2}$ ) and eigenvectors $v_{1}, v_{2}$ of the STs were calculated. The primary image orientation can be obtained from the larger eigenvector, hence the planar angle

$$
\phi_{S T}=\arctan \left(\frac{v_{1, y}}{v_{1, x}}\right)
$$

of $v_{1}$ describes the sought-for orientation in a pixel. 


\section{Creating a histological voxel space}

In order to make histological and DTI data comparable, the histological data had to be converted from their original spatial resolution to the DTI voxel resolution. This was done by partitioning the histological sections into subimages measuring $(340 \times 340) \mu \mathrm{m}$ and using different operations to downsample the histological values determined in each pixel to subimage or "voxel" values. The gray level of a voxel was obtained by taking the average over the subimage pixel values. Via the expression: 1 - gray level, it served as a measure of staining intensity in the statistical analysis. For calculation of voxel orientations, simple averaging was not applicable due to the periodicity of circular data. Instead, for each voxel a 180 bin $\left(=1^{\circ}\right.$ angular resolution) histogram of all pixel orientations was created, normalized (division by the subimage pixel number) and denoised by convolution with a Gaussian window of 23 degrees full width at half maximum (FWHM). The smoothed histograms were then fitted with mixtures of von Mises distributions, which closely resemble wrapped normal distributions (Lee 2010). The fitting of three von Mises components on each smoothed histogram $h$ was conducted using the following algorithm:

Find the maximum $\left(x_{\max }, y_{\max }\right)$ of $h$. Take it as center $\theta$ and amplitude $d$ of a von Mises component $\frac{d}{f_{\theta, \kappa}\left(x_{\max }\right)} f_{\theta, \kappa}$ with $f$ the von Mises probability density function. Due to the axial nature of the data $\left(f\left(0^{\circ}\right)=f\left(180^{\circ}\right)\right), f$ was defined on the half instead of the full circle. Determine the dispersion parameter $\kappa$ of that component by minimizing the square error in a local neighborhood around $\theta$, i.e. solve the optimization problem

$$
\min _{\kappa} \sum_{x=\theta-42}^{\theta+42}\left(h(x)-\frac{d}{f_{\theta, \kappa}\left(x_{\max }\right)} f_{\theta, \kappa}(x)\right)^{2}
$$

Replace $h$ with the residual $h(x)-\frac{d}{f_{\theta, \kappa}\left(x_{\max }\right)} f_{\theta, \kappa}$, repeat.

The algorithm was implemented in Matlab 2012, making use of a freely available toolbox for circular statistics (Berens 2009). In summary, this algorithm returned the values of theta, kappa, and amplitude for 
each of three von Mises components. The theta values should indicate in descending order the directions of the three most prominent fiber directions in a histological voxel. The first of these values was considered as the primary orientation $\Phi_{S T}$ of a voxel and was used to determine the orientation differences to DTI-results. In theory, this method was capable of accurately identifying three directions within an image. In practice however, only the primary and the secondary orientations reliably represented fiber directions (Fig. 1). From the kappa value, which indicates the "narrowness" of a component, its width was inferred via $1-\frac{\kappa}{\kappa_{\max }}$, with $\kappa_{\max }$ the maximum over all analyzed voxels. Both amplitude and width of the primary component are measures of how peaked, i.e. anisotropic, the fiber orientation distribution is. Therefore, we expected these values to be correlated to FA as well as the orientation match with DTI.

\subsection{Alignment}

The MRI and the histological volume were coregistered using a 3D affine transformation, a combination of rotations, translations, and scales. Since the tissue was sectioned precisely along the xy-plane of the MRI scan, a rotation in the $x z$ and yz-planes was unnecessary. Furthermore, as the histological volume was not $3 \mathrm{D}$ but a series of $2 \mathrm{D}$ sections, the scaling and translation along the z-axis was replaced by a (manually conducted) assignment between histological sections and MRI z-slices. The MRI voxel resolution was $340 \mu \mathrm{m}$ in each direction while neighboring sections were $300 \mu \mathrm{m}$ apart from each other $(60 \mu \mathrm{m}$ thickness times every 5 th section stained), so this was approximately a 1:1 assignment with each 10th zslice mapped onto two consecutive histological sections. For the resulting section-slice pairs, the remaining operations were one rotation (xy-plane), two translations ( $x$ and $y$-direction) and one scaling (xy-direction - exploration revealed that separate scaling in $\mathrm{x}$ and $\mathrm{y}$-direction was unnecessary). These operations were performed using a custom-made graphical user interface in Matlab 2012a where the section images could be gradually moved onto the respective slices. In this way, a transformation, represented 

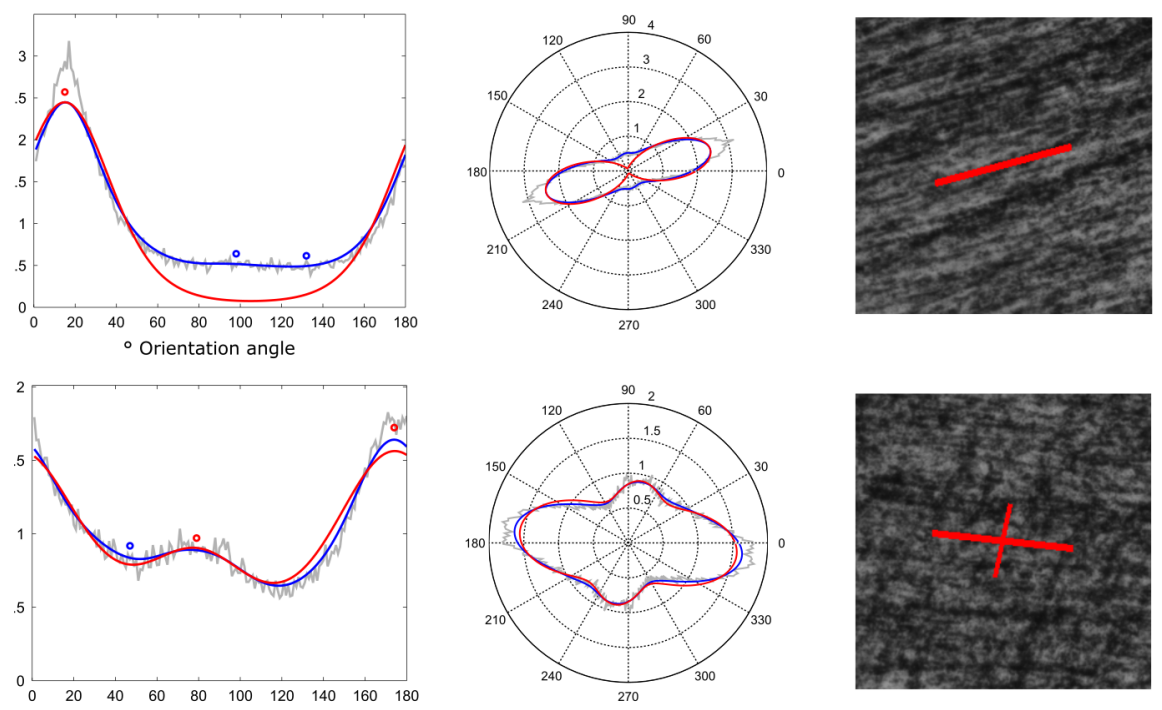

Figure 1: Histological fiber orientation distribution with fitted von Mises mixture for two exemplary voxels. Left: Normalized histograms of structure tensor orientations of all pixels within a voxel (gray line), smoothed with a Gaussian window (blue line) and fitted using a mixture of three von Mises probability density functions (red line). The central values of the von Mises components were the resulting histological orientations in a voxel (circles). Middle: Same histograms as polar plots. Right: High amplitude voxel orientations (corresponding to red circles) visualized on corresponding histological tile. a: Example of 1-orientation voxel, b: 2-orientation voxel.

as a $3 \times 3$ matrix in homogeneous coordinates, was determined for each section-slice pair, translating histological into MRI coordinates and vice versa.

\subsection{Diffusion tensor orientations}

In order to obtain planar orientation angles from the diffusion tensors, in each voxel the eigenvector $w_{\max }$ corresponding to the largest eigenvalue was projected into the sectioning plane (since the sectioning plane was the xy-plane of the MRI volume, this was achieved by discarding the 
$\mathrm{z}$-coordinate). From this projection, the orientation angle was obtained as

$$
\Phi_{D T}=\arctan \left(\frac{w_{\max , y}}{w_{\max , x}}\right)
$$

Of course, the projection is shorter the more perpendicularly $w_{\max }$ is oriented towards the xy-plane, resulting in information loss. The same holds for the histological data, where orthogonally cut fibers appear as dots. To alleviate this problem, only voxels with an out-of-plane angle of maximally 45 degrees were used for further analyses.

\subsection{Statistical evaluation}

\section{Orientation differences}

The difference between DT- and ST-based voxel orientations in axis angles was calculated as

$$
d\left(\Phi_{D T}, \Phi_{S T}\right)=\min \left(\left|\Phi_{D T}-\Phi_{S T}\right|, 180^{\circ}-\left|\Phi_{D T}-\Phi_{S T}\right|\right)
$$

The central tendency of these differences across voxels was measured in terms of arithmetic mean and median. This was done for the entire volume as well as individually for gray/white matter and for different ranges of FA.

\section{Correlations and regression}

Correlations across voxels were calculated between a range of values derived from DTI (FA, MD, RD), histology (staining intensity, amplitude and width parameters of the von Mises fit), or both combined (orientation difference).

Additionally, multiple linear regression was used to predict FA, MD, and $\mathrm{RD}$ from the histology-based variables. The overall amount of predictability is reported as percentage of explained variance $\left(R^{2}\right)$.

As the intensity of the myelin stain varied between the sections due to technical reasons, calculation of correlations and regressions over the entire 
volume would not have led to reliable results. Instead, these calculations were performed for each section individually. We report the mean of the section results, weighted with the number of voxels in each section.

\section{Results}

\subsection{Sample sizes}

A total of 65 histological sections were mapped onto 60 volume slices. In voxel space (Cartesian coordinate system with $340 \mu \mathrm{m}$ unit length), this provided a sample of $N_{\text {Pair }}=221681$ voxels for which both a diffusion tensor and histological structure tensors were available. $6.13 \%$ of all voxels were discarded due to tissue damage and $41.11 \%$ for not including stained material. Of the remaining voxels, $53.68 \%$ were within the threshold for the out-of-plane angle (see 2.6), leading to a subset of $N=62782$ voxels as the data set for subsequent analyses. Out of these, $27.6 \%$ of voxels were located within gray matter, and $72.4 \%$ within white matter.

\subsection{Orientation differences}

Throughout all analyzed voxels, the average difference between MRI diffusion tensor $\left(\mathrm{MRI}_{\mathrm{DT}}\right.$ ) and histology structure tensor (Hist $\mathrm{ST}_{\mathrm{ST}}$ ) orientations was $14.25^{\circ}$, with a median of $9.01^{\circ}$. Differences were generally larger in gray matter (mean $19.51^{\circ}$, median $\left.11.25^{\circ}\right)$ than in white matter $\left(12.25^{\circ}\right.$, $\left.8.34^{\circ}\right)$. In both tissue types, the distribution of orientation differences resembled a power law, with most voxels showing differences smaller than 10 degrees, and with a long distribution tail (Fig. 2). Mathematically, this was best described with a generalized Pareto distribution (explained variance $R_{W M}^{2}=.996, R_{G M}^{2}=.960$ ). This implies that mean orientation differences (being sensitive to large values in the distribution tail) might give an inflated interpretation of central tendency, which is better reflected by the remarkably smaller difference medians. In gray matter, a deviation from this distribution could be observed near $90^{\circ}$, revealing an increased 


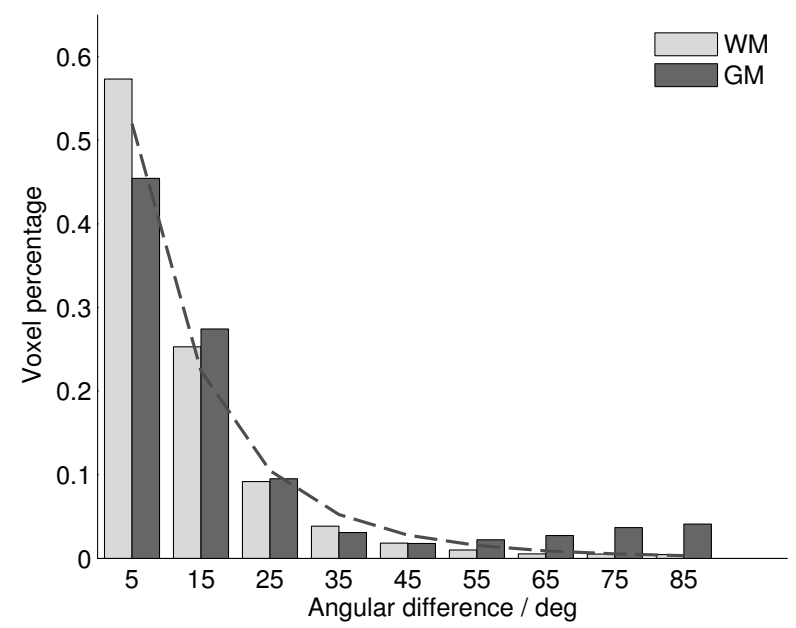

Figure 2: Histogram of angular differences between $\mathrm{MRI}_{\mathrm{DT}}$ and Hist $\mathrm{ST}_{\mathrm{ST}}$ orientations over all analyzed voxels for white and gray matter. Dashed line: Histogram fit with generalized Pareto distribution.

tendency towards orthogonally oriented $\mathrm{MRI}_{\mathrm{DT}}$ and Hist $\mathrm{ST}_{\mathrm{T}}$ orientations. Most of these voxels were arranged in cortical bands, where stained fibers ran parallel to the cortical surface, while the diffusion tensors fanned out radially from white matter (Fig. 3a, 6c).

This phenomenon accounted for most of the difference in orientation match between gray and white matter: When truncated at $50^{\circ}$, the mean difference in white $\left(10.85^{\circ}\right)$ and gray $\left(11.74^{\circ}\right)$ matter was much more similar.

Throughout both white and gray matter, voxels with multiple fiber orientations, due to e.g. crossings, showed remarkable differences between histological and DTI orientations, as shown in Figure 3. In these voxels, the diffusion tensors often represented either a mixture of the existing fiber orientations (Fig. 3b for relatively low angle crossings in WM) or a selection of one of the two directions (Fig. 3a for the orthogonal crossings in gray matter where DTI picks the radial orientation). Voxels with single fiber orientations (Fig. 3c) are well represented by the DTI orientation. 
Figure 4 shows the spatial distribution of orientation differences for one exemplary section.

\section{Relation of FA to angular differences}

Plotting the angular difference over tissue type and FA, we found that, as expected, FA depended largely on the tissue type (WM and GM) and that the angular difference was a function of both tissue type and FA (Fig. 5). In white matter, most voxels showed FA values between .25 and .45. For an FA greater than .3, the angular difference was smaller than 11 degrees. For FA values lower than that, the orientation agreement with histology decreased remarkably and monotonically with decreasing FA. In gray matter, mostly FA values between .15 and .2 were observed. Here the angular difference was between 15 and 21 degrees which is more than the average white matter difference, but less than the white matter difference at this FA level. For FA values lower than .15, the orientation match again was very poor, eventually approaching chance level for an FA of .05.

The relation between FA and orientation difference was additionally explored visually by plotting histological and DT orientations on FA maps (Fig. 6). Overall, it clearly appears that good orientation agreement is achieved where FA is high. However, there are also areas observable where this was not the case, particularly at the transition from white to gray matter. There, FA often decreased significantly (cf. Miller et al. 2011) while the orientation difference remained small.

\subsection{Correlation and regression results}

Pairwise correlations between different parameters obtained from the MRI and the histological volume were calculated to quantify linear connections between the two data sources, particularly between the scalar measures of DTI and myeloarchitectonic characteristics. An overview of the correlation results is given in Table ??. Fractional anisotropy was strongly correlated to the staining intensity (.487), the primary and secondary von 

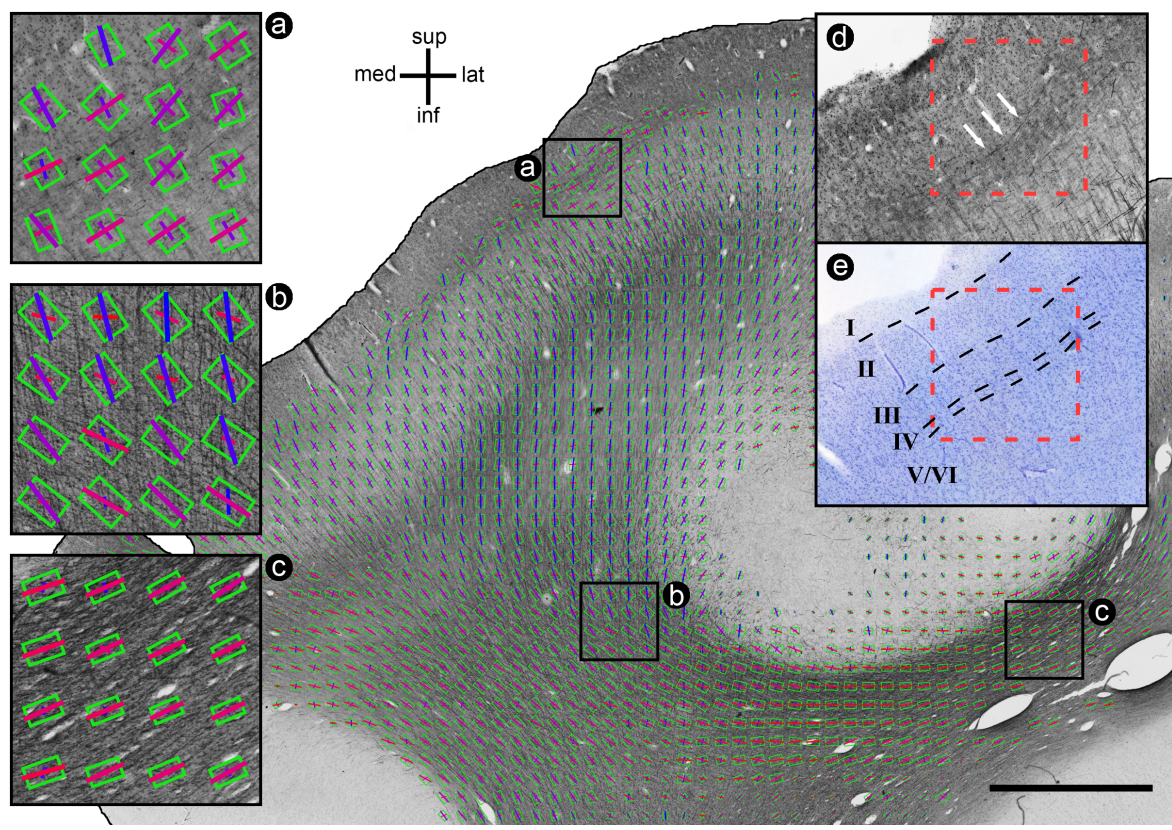

Figure 3: Exemplary histological section with $\mathrm{MRI}_{\mathrm{DT}}$ and Hist $\mathrm{ST}_{\mathrm{ST}}$ orientations. Diffusion tensors are coded by oriented green rectangles, where rectangle aspect ratio indicates fractional anisotropy (more elongated $=$ higher FA). Hist ${ }_{\mathrm{ST}}$ orientations are coded by bars. The color of the bars indicates the respective orientation (lateral-medial = red; inferiorsuperior = blue). Size of DT rectangles and ST bars is proportional to DT projection length. Scale bar: $3 \mathrm{~mm}$. a: Voxels in which myelin orientations are parallel to the cortical surface, DT orientations orthogonal. b: Fiber crossing. FA is low and the DT orientation is in between the primary and secondary histological orientation. c: 1-orientation fiber pathway. FA is high, the secondary histological orientations are very small, and there is a good match of primary histological and DT orientation. d: area a) without DT and ST information. Arrows indicate the tangential fibers determining the ST orientations. e: Classification of cortical layers in an area corresponding to area a) in a neighboring Nissl-stained section. 


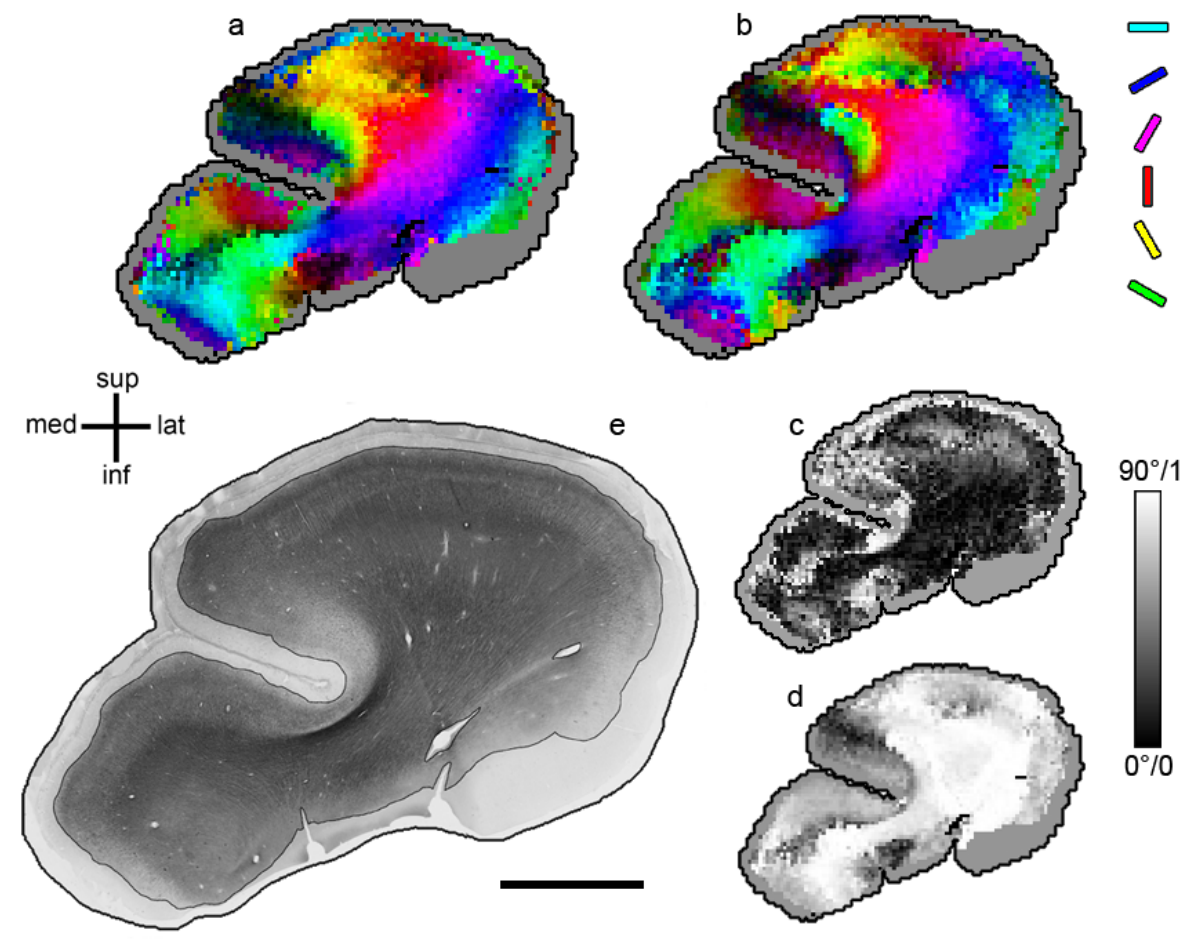

Figure 4: $\mathrm{MRI}_{\mathrm{DT}}$ and Hist $\mathrm{ST}_{\mathrm{S}}$ orientations in one exemplary section. a: Diffusion tensor orientations projected into the sectioning plane. Orientation angle is coded as hue (with the full color spectrum to optimize orientation contrast), projection length as brightness in HSB space. b: Primary structure tensor orientations, same color code as a. c: Mapping of angular difference between $\mathrm{a}$ and $\mathrm{b}$ in axis angles (ranging from $0^{\circ}$ to $90^{\circ}$ ). d: Mapping of projection length of diffusion tensors into the sectioning plane (ranging from 0 to 1 ). Note that a short projection length is a possible but not the only source of high angular difference in c. e: Original section with stained area emphasized. In a-d, the parts without stained fiber material are displayed in gray. Size a-d: 1 image pixel equals 1 voxel $(340 \mu \mathrm{m})$, e: scale bar $=5 \mathrm{~mm}$. 


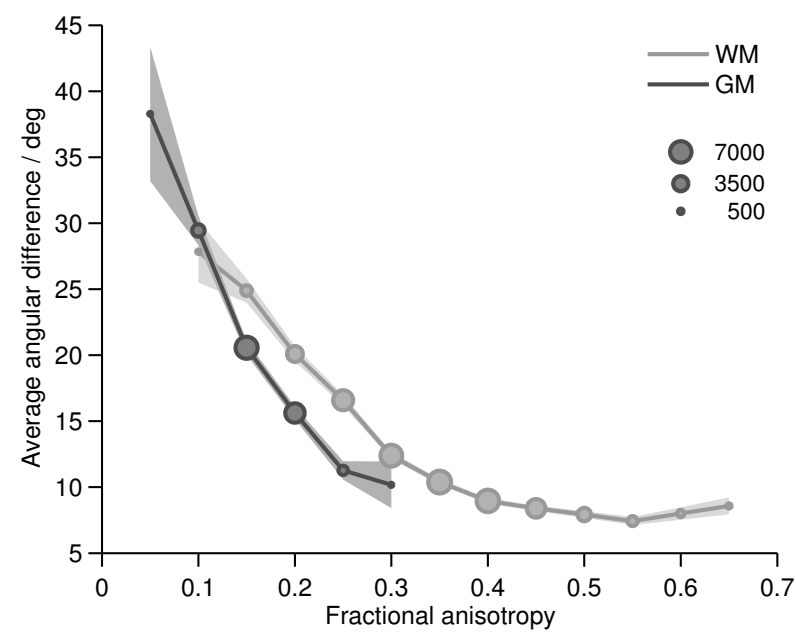

Figure 5: Average difference between main $\mathrm{MRI}_{\mathrm{DT}}$ and Hist $\mathrm{ST}_{\mathrm{T}}$ orientations in gray (dark line) and white (light line) matter as a function of FA. Marker size linearly represents the sizes of the voxel subsamples of the FA bins. FA bins with less than 100 voxels are not displayed. Confidence interval: $1.96 *$ standard error.

Mises components' amplitudes (.478, -.371), and to some degree with the orientation difference $d\left(\Phi_{D T}, \Phi_{S T}\right)$ (-.303). The correlation patterns of mean diffusivity and radial diffusivity were similar to that of FA but with opposite signs. Compared to FA, MD and RD correlated more strongly, but negatively, with staining intensity $(-.542, .-554)$ and the amplitude of the secondary orientation $(.397, .405)$, but less strongly with the main orientation amplitude (-.309, -.374).

Aside from its correlation with FA and MD, the histological FOD as represented by the amplitude of the primary von Mises component showed a moderate correlation with the orientation difference (-.325). This means that the orientations of diffusion tensors and histology tended to be more similar when the FOD was unimodal. The width of the secondary as well as the amplitude and width parameters of the tertiary component showed no strong correlations with DTI-related measures. Predicting FA, MD, and $\mathrm{RD}$ from the histological measures (staining intensity, FOD parameters) by means of linear regression, the percentages of explained variance $\left(R^{2}\right)$ found were .472 for FA, .449 for MD, and .483 for RD. 


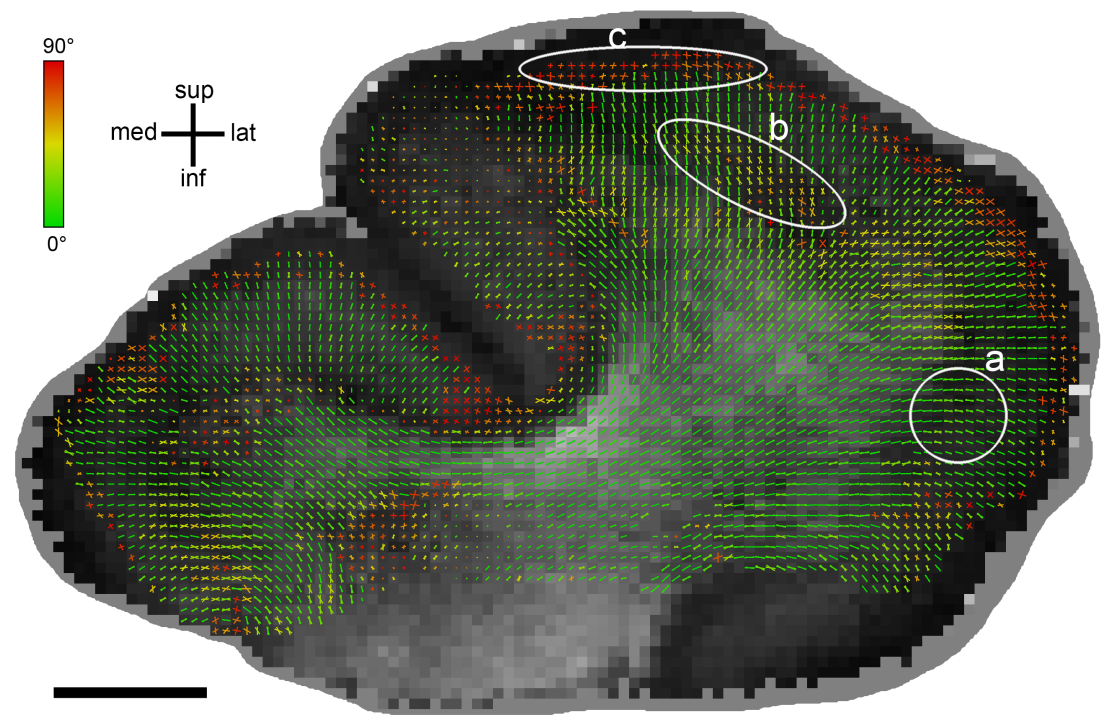

Figure 6: Differences between $\mathrm{MRI}_{\mathrm{DT}}$ and Hist $\mathrm{ST}_{\mathrm{S}}$ orientations displayed on an FA map. $\mathrm{MRI}_{\mathrm{DT}}$ and HistsT orientations are plotted in a color range from green (for orientations that perfectly agree) to red (for orientations that differ by 90 degrees). Bar length reflects the projection length of the diffusion tensor in the sectioning plane. There is a generally good histology-DTI agreement for high FA values, especially in white matter. For lower FA, particularly when entering gray matter, there are three cases: a. Good agreement in case of predominant radial orientations, $\mathrm{b}$. Moderate to weak agreement, $\mathrm{c}$. Perpendicular histology-DT orientedness. Here, voxels are located well within gray matter and run in bands parallel to the cortical surface. Scale bar: $5 \mathrm{~mm}$.

\section{Discussion}

The aim of this study was to investigate the microstructural basis of diffusion-weighted MRI. We were interested in the quantitative relationship between fiber orientations and scalar characteristics as inferred from a dMRI volume and a histological analysis of the same tissue probe. To this end, fiber orientation distributions from a large number of digitized myelin-stained sections were derived using the structure tensor approach (Schmitt and Birkholz 2011, Budde and Frank 2012, Budde and Annese 2013). While obtained from post-mortem data, we would expect our findings to be generalizable to the in-vivo case. Although fixed ex-vivo 
Table 1: Correlation table of histological and dMRI features

\begin{tabular}{|c|c|c|c|c|c|c|c|c|}
\hline & Diff $_{\text {ST-DT }}$ & FA & MD & RD & Stain & $\mathrm{Ampl}_{1}$ & Width $_{1}$ & $\mathrm{Ampl}_{2}$ \\
\hline $\operatorname{Diff}_{\text {ST-DT }}$ & 1 & -0.303 & 0.293 & 0.315 & -0.23 & -0.325 & 0.293 & 0.158 \\
\hline FA & -0.303 & 1 & -0.76 & -0.854 & 0.487 & 0.478 & -0.394 & -0.371 \\
\hline MD & 0.293 & -0.76 & 1 & 0.983 & -0.542 & -0.309 & 0.222 & 0.397 \\
\hline $\mathrm{RD}$ & 0.315 & -0.854 & 0.983 & 1 & -0.554 & -0.374 & 0.285 & 0.405 \\
\hline Stain & -0.23 & 0.487 & -0.542 & -0.554 & 1 & 0.313 & -0.247 & -0.285 \\
\hline $\mathrm{Ampl}_{1}$ & -0.325 & 0.478 & -0.309 & -0.374 & 0.313 & 1 & -0.977 & -0.105 \\
\hline Width $_{1}$ & 0.293 & -0.394 & 0.222 & 0.285 & -0.247 & -0.977 & 1 & -0.086 \\
\hline $\mathrm{Ampl}_{2}$ & 0.158 & -0.371 & 0.397 & 0.405 & -0.285 & -0.105 & -0.086 & 1 \\
\hline
\end{tabular}

Features are: Angular difference between primary $\mathrm{Hist}_{\mathrm{ST}}$ and $\mathrm{MRI}_{\mathrm{DT}}$ orientation

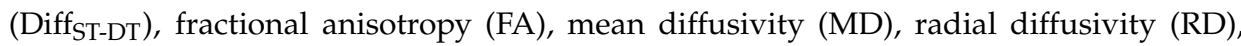
staining intensity (1 - voxel gray level), amplitude and width of the primary, amplitude of the secondary Hist ST $_{\text {T }}$ orientation. Mutual Pearson correlations were calculated separately for each of 65 sections and averaged weighted by the respective voxel counts (standard deviations were always smaller than 0.2 ). Correlations with an absolute value greater than 0.3 are displayed in bold font.

tissue has different $M R$ relaxation parameters (reduced $T_{1}$ and $T_{2}$ ) and reduced water diffusion compared to in-vivo tissue, diffusion anisotropy, the crucial marker for neurite orientation and microstructure estimation, remains intact. This means that our correlation, regression and orientation findings should translate well, even if higher absolute values of scalar measures can be expected in-vivo.

\subsection{Orientations}

The angular differences between diffusion tensor and histology-based fiber orientations were taken as an indicator of the goodness of fit of the diffusion tensor as a model of different white matter configurations and gray matter myeloarchitecture.

Our analysis revealed that these differences followed a power law (Pareto) distribution, being smaller than 10 degrees in approximately half of the voxels. Given measurement inaccuracies, these values probably 
represent a close to ideal match between DT and fiber directions, confirming that (at high spatial MRI resolution) the diffusion tensor is a very accurate model of fiber orientation in about half of the analyzed tissue. Visual exploration (cf. Fig. 3) as well as the correlation between unimodal histological FODs and the observed orientation match confirmed that, as expected, this was particularly the case in areas dominated by homogeneously oriented fiber pathways.

However, roughly one third of voxels showed an orientation mismatch between 10 and 45 degrees. While angular differences of less than $10^{\circ}$ are most likely caused by measurement inaccuracies, these higher values suggest additional sources of mismatch. We saw that in voxels containing crossing fibers, diffusion tensors tended to shift away from the primary towards the secondary histologically derived orientations. The amount of this shift was related to the prominence of the secondary orientations, as reflected by the correlation between angular differences and FOD parameters. Recently, there have been extensive considerations about the best approach to cope with this issue. One important way forward is to opt for more complex models based on high angular resolution diffusion imaging (HARDI), such as q-ball imaging (Tuch 2004), diffusion spectrum imaging (DSI) (Wedeen et al. 2005), or spherical deconvolution (Tournier et al. 2007, 2004) in order to model the whole orientation profile within a voxel rather than only its principal direction. Likewise, a better spatial resolution should reduce the amount of voxels with problematic fiber crossings. The histological structure tensor approach produces fiber orientation distributions of high angular resolution at a "voxel size" which can be chosen arbitrarily. It would therefore be suited to investigate both the performance of complex dMRI models and the effect of different spatial resolutions on dMRI results in future studies.

There were also voxels which displayed angular differences of more than 45 degrees. This cannot be accounted for by crossing fibers only, as such an averaging should give values of maximally $45^{\circ}$. Thus it can only occur when either the fiber material has no clear orientational structure or when DT and ST are based on different anatomical structures. In white 
matter, this was only observed in a very small proportion of voxels $(3.3 \%)$. In gray matter however, this proportion was much larger (13.6\%) and the distribution of angular differences showed a second peak at about 90 degrees, indicating that in a sustantial subpopulation of voxels DT and ST orientations were orthogonal to each other. These voxels were found mostly at intermediate depths within gray matter, arranged in bands running parallel to the cortical surface (Fig. 3a, 6c). In these bands, the histological orientations were tangential, caused by myelinated horizontal axons corresponding to the bands of Baillarger (Nieuwenhuys 2013). The DT orientations in these regions, however, were oriented radially. The organized structure of pyramidal cell apical dendrites is a likely basis for these diffusion tensor orientations and should be invisible to the structure tensors due to the lack of myelin in dendrites. Such prominent radial bundles of dendrites can be found throughout gray matter and are most pronounced between layers III and V (Peters et al. 1997). In layer IV and $\mathrm{Vb}$, these radial dendritic bundles cross the horizontally oriented bands of Baillarger which could explain the orthogonal orientation mismatch between DT and ST. This issue should be further elaborated in future studies using alternative histological labels, such as lipophilic dyes (Budde and Frank 2012) or immunohistochemical staining of different neuronal or glial compounds. Such approaches could also enable the study of orientation distributions in layers I and II, which are weakly myelinated (Nieuwenhuys 2013, Vogt and Vogt 1919, Sanides 1962).

Overall, these voxels with orthogonal DT-ST orientations were the main reason for the worse DT-ST match in gray than in white matter. Excluding them from the analysis, diffusion tensors were only marginally less aligned to stained fiber directions than in white matter. This points towards a good applicability of DTI not only to white matter but also at least to lower cortical layers revealing the characteristic radial structure of neurites here. This seems to run counter to the common experience with in-vivo diffusion MRI, but one has to keep in mind that the spatial resolution of our scan was much higher than what is currently used for in-vivo measurements. Therefore, an increase in diffusion MRI resolution to values significantly 
below one millimeter can considerably enlarge the scope of diffusion MR to include the analysis of gray matter structures. Due to the use of a myelin staining approach, we can at present not make predictions about DTI performance in the supragranular cortical layers. However, the fact that diffusion tensor orientations were much more variable in these layers than in infragranular layers suggests that DTI is still not a very well suited model for all cortical layers. This assumption is backed by recent studies on gray matter architecture using high resolution dMRI post-mortem, all of which employed dMRI models more complex than DTI (Bastiani et al. 2013, Kleinnijenhuis et al. 2013, Leuze et al. 2014).

\subsection{Scalar measures}

We investigated the relation of FA, MD, and RD with white matter microstructure and gray matter myeloarchitecture. We found a group of medium to strong correlations between diffusion MRI measures and myelin histology measures: FA was positively linked with high staining intensity, a narrow peak in the histological FODs and a good agreement between the orientations of the diffusion tensor and the stained fibers. Conversely, high values of both mean diffusivity and radial diffusivity were linked to bimodal or diffuse histology FODs, weakly matching orientations, and a low staining intensity. These interrelations were strong enough to explain about $50 \%$ of the variance of FA, MD, and RD from histological variables in a multiple linear regression, which confirms and to some extent quantifies the role of myelin as an important modulator of diffusion anisotropy and overall diffusivity (Beaulieu 2002).

Comparing the different correlation patterns of FA, MD, and RD, we found that FA was related most strongly to the orientational specificity of the FODs in the histological data whereas MD predominantly reflected staining intensity. This confirms that, as intended, FA is a measure of the anisotropy of fiber distributions, and MD of the overall amount of diffusion-restricting material (in this case myelin sheaths). RD showed a 
pattern somewhat midway between these extremes with a high correlation to both FOD characteristics and staining intensity.

However, the intensity of myelin stains does not only depend on the amount of myelin but also on the staining protocol and the achieved quality of the staining. First, the quality of myelin staining we were able to achieve was suboptimal compared to what can be obtained in a fresh sample, fixed, sectioned and stained soon after death. The tissue probe used in this study was fixed for about 1 year when it was stained, resulting in a paler stain than what is usually achieved immediately post-mortem. Even though unintended, for the purpose of identifying directions of individual fibers the relatively sparse staining proved helpful. Second, the Gallyas stain used here is known to label myelin well and produce clearly interpretable histology images but also to be less consistent than other protocols, i.e. it will often show a substantial amount of variance within and in between tissue probes. We have corrected for section-to-section variability by performing statistics sectionwise and pooling statistics over sections. However, the varying staining intensity within each section has to be seen as a possible noise source in our correlation results. These concerns for the interpretability of staining intensity apply much less to orientations because the structure tensor analysis is relatively robust to absolute image intensity and its global variations.

The analysis of orientation differences at different FA ranges revealed that FA had a high predictive value for the angular difference between ST and DT orientations. This gives FA some credibility as an indicator for the reliability of DTI results; in particular, it justifies its usage as a stopping criterion in tractography algorithms. However, FA must be evaluated differently for white and gray matter. In white matter, FA values above 0.3 seemed sufficient for a good ST-DT orientation match, whereas in gray matter this was already the case for values of 0.2 (within the confines discussed above). This was confirmed by investigating the white-gray matter boundary (Fig 6): at the transition from white to gray matter, a decrease of FA values was often observed although both diffusion tensors and the stained fibers still followed the same pathways. This could have 
potential practical implications for DTI tractography algorithms using FA thresholds as a stopping criterion. Our results indicate that, at high spatial resolutions, thresholds suitable for white matter tractography might be overly strict when applied to the deeper gray matter layers. Therefore, it could be advisable to use tractography algorithms in conjunction with a gray-white matter segmentation, adjusting FA thresholds across the gray-white matter boundary. In our post-mortem sample, 0.3 would be a suitable FA threshold for white matter and 0.2 for gray matter.

\subsection{Conclusion}

DTI is one of the simplest mathematical models available for diffusion weighted imaging, yet by far the most commonly used. We verify quantitatively here that structures which are simple enough to be modeled by diffusion tensors (individual coherently aligned fibers) are indeed described very accurately. Hence when analyzing strong, preferably unidirectional fiber pathways, DTI still appears to be the best choice due to its short acquisition time and low sensitivity to noise which generally comes with simple models. Furthermore, FA, MD, and RD were confirmed as sensitive measures of microstructural tissue characteristics, particularly being related to density and dispersion of myelinated fibers. However, complex fiber architecture with more than one principal fiber orientation within individual voxels cannot be sufficiently modeled using DTI. We found that in such cases diffusion tensor orientations represent either a mixture of the existing fiber orientations for relatively low angle crossings (often occurring in WM) or a selection of one of the two directions (for the orthogonal crossings typical of certain gray matter layers).

We provide new insights into the neuroanatomical basis of diffusion MRI in gray matter, where it is recently applied more often as spatial resolution increases to the required submillimeter level. In the deeper cortical layers (V, VI), diffusion tensor orientations matched myeloarchitecture almost as well as in white matter, accurately modeling cortical afferent and efferent fibers. This implies that, at high spatial resolution, 
FA thresholds might have to be adapted in order to conduct tractography across the white-gray matter boundary. In layers V to III, diffusion tensors were mostly oriented radially towards the cortical surface reflecting unmyelinated structure, presumably bundles of apical dendrites, which are orthogonal to tangential myelinated fibers. Although DTI followed these structures robustly, this selectivity for a subset of oriented neuroanatomical structures indicates that multi-orientation models are more appropriate in superficial cortical layers.

In future studies, the approach of validating dMRI results with histologically obtained FODs can be extended into a number of directions. Due to its adjustable angular and spatial resolution, the structure tensor technique could be used to investigate complex dMRI models as well as the effect of different spatial resolutions on dMRI results. Furthermore, tissue clearing methods have gained some attention in recent years for their ability to create transparent blocks of brain tissue which are compatible with immunohistochemistry (Dodt et al. 2007, Chung and Deisseroth 2013). With the appropriate protocols and ways to obtain digitized data sets, one could use these methods to derive 3D structure tensors from tissue probes. This would make dMRI-ST comparisons even more viable and open up new possibilities to histologically validate dMRI tractography.

\section{References}

Assaf, Y., Alexander, D. C., Jones, D. K., Bizzi, A., Behrens, T. E., Clark, C. A., Cohen, Y., Dyrby, T. B., Huppi, P. S., Knoesche, T. R., LeBihan, D., Parker, G. J., and Poupon, C. (2013). The CONNECT project: Combining macro- and micro-structure. NeuroImage, 80:273-282.

Assaf, Y. and Basser, P. J. (2005). Composite hindered and restricted model of diffusion (CHARMED) MR imaging of the human brain. NeuroImage, 27(1):48-58.

Bartsch, H., Maechler, P., and Annese, J. (2012). Automated determination 
of axonal orientation in the deep white matter of the human brain. Brain connectivity, 2(5):284-290.

Basser, P., Mattiello, J., and LeBihan, D. (1994). Estimation of the effective self-diffusion tensor from the NMR spin echo. Journal of Magnetic Resonance, Series B, 103:247-254.

Bastiani, M., Oros-Peusquens, A., Brenner, D., Moellenhoff, K., Seehaus, A., Celik, A., Felder, J., Matusch, A., Galuske, R., Bratzke, H., Shah, N., Goebel, R., and Roebroeck, A. (2013). Cortical fiber insertions and automated layer classification in human motor cortex from 9.4T diffusion MRI. In ISMRM 21st Annual Meeting E Exhibition, page 2124, Salt Lake City, UT.

Beaulieu, C. (2002). The basis of anisotropic water diffusion in the nervous system - a technical review. NMR in Biomedicine, 15:435-455.

Berens, P. (2009). CircStat: a MATLAB toolbox for circular statistics. Journal of Statistical Software, 31(10):1-21.

Bigün, J. and Granlund, G. (1987). Optimal orientation detection of linear symmetry. In Proceedings of the IEEE First International Conference on Computer Vision, pages 433-438, London. IEEE Computer Society Press.

Budde, M. D. and Annese, J. (2013). Quantification of anisotropy and fiber orientation in human brain histological sections. Frontiers in Integrative Neuroscience, 7(3):10-3389.

Budde, M. D. and Frank, J. A. (2012). Examining brain microstructure using structure tensor analysis of histological sections. NeuroImage, 63(1):1-10.

Catani, M., Howard, R., Pajevic, S., and Jones, D. (2002). Virtual in vivo interactive dissection of white matter fasciculi in the human brain. NeuroImage, 17:77-94.

Catani, M. and Thiebaut de Schotten, M. (2008). A diffusion tensor imaging tractography atlas for virtual in vivo dissections. Cortex, 44(8):1105-1132. 
Choe, A. S., Gao, Y., Li, X., Compton, K. B., Stepniewska, I., and Anderson, A. (2011). Accuracy of image registration between MRI and light microscopy in the ex vivo brain. Magnetic Resonance Imaging, 29:683 692.

Chung, K. and Deisseroth, K. (2013). CLARITY for mapping the nervous system. Nature methods, 10(6):508-513.

Dodt, H.-U., Leischner, U., Schierloh, A., Jährling, N., Mauch, C. P., Deininger, K., Deussing, J. M., Eder, M., Zieglgänsberger, W., and Becker, K. (2007). Ultramicroscopy: three-dimensional visualization of neuronal networks in the whole mouse brain. Nature methods, 4(4):331-336.

Economo, C. and Koskinas, G. N. (1925). Die Cytoarchitektonik der Hirnrinde des erwachsenen Menschen. J. Springer.

Gallyas, F. (1979). Silver staining of myelin by means of physical development. Neurological Research, 1(2):203-9.

Hagmann, P., Cammoun, L., Gigandet, X., Meuli, R., Honey, C. J., Wedeen, V. J., and Sporns, O. (2008). Mapping the structural core of human cerebral cortex. PLoS biology, 6(7):e159.

Jenkinson, M., Beckmann, C. F., Behrens, T. E., Woolrich, M. W., and Smith, S. M. (2012). FSL. NeuroImage, 62(2):782-790.

Jeurissen, B., Leemans, A., Tournier, J., Jones, D. K., and Sijbers, J. (2013). Investigating the prevalence of complex fiber configurations in white matter tissue with diffusion magnetic resonance imaging. Human Brain Mapping, 34(11):2747-2766.

Jones, D. K. (2010). Precision and accuracy in diffusion tensor magnetic resonance imaging. Topics in Magnetic Resonance Imaging, 21(2):87-99.

Jones, D. K., Knösche, T. R., and Turner, R. (2013). White matter integrity, fiber count, and other fallacies: The do's and don'ts of diffusion MRI. NeuroImage, 73:239-254.

Kanaan, R., Kim, J., Kaufmann, W., Pearlson, G., Barker, G., and McGuire, 
P. (2005). Diffusion tensor imaging in schizophrenia. Biological Psychiatry, 58:921-929.

Kemao, Q. and Asundi, A. (2002). Characterizing young's fringes' orientation and spacing by fourier transform and radon transform. Optics $\mathcal{E}$ Laser Technology, 34(7):527-532.

Kleinnijenhuis, M., Zerbi, V., Küsters, B., Slump, C. H., Barth, M., and van Cappellen van Walsum, A.-M. (2013). Layer-specific diffusion weighted imaging in human primary visual cortex in vitro. Cortex, 49(9):25692582.

Lee, A. (2010). Circular data. Wiley Interdisciplinary Reviews: Computational Statistics, 2(4):477-486.

Leemans, A. and Jones, D. K. (2009). The B-matrix must be rotated when correcting for subject motion in DTI data. Magnetic Resonance in Medicine, 61(6):1336-1349.

Leergaard, T., White, N., de Crespigny, A., Bolstad, I., D'Arceuil, H., Bjaalie, J., and Dale, A. (2010). Quantitative histological validation of diffusion MRI fiber orientation distributions in the rat brain. PLoS One, 5:e8595.

Lefebvre, A., Corpetti, T., and Moy, L. H. (2011). Estimation of the orientation of textured patterns via wavelet analysis. Pattern Recognition Letters, 32(2):190-196.

Leuze, C. W., Anwander, A., Bazin, P.-L., Dhital, B., Stüber, C., Reimann, K., Geyer, S., and Turner, R. (2014). Layer-specific intracortical connectivity revealed with diffusion MRI. Cerebral Cortex, 24(2):328-339.

Lin, C., Tseng, W., Cheng, H., and Chen, J. (2001). Validation of diffusion tensor magnetic resonance axonal fiber imaging with registered manganese-enhanced optic tracts. NeuroImage, 14:1035-1047.

Medina, D. and Gaviria, M. (2008). Diffusion tensor imaging investigations in Alzheimer's disease: the resurgence of white matter compromise in 
the cortical dysfunction of the aging brain. Neuropsychiatric Disease and Treatment, 4:737-742.

Michelet, F., Da Costa, J.-P., Lavialle, O., Berthoumieu, Y., Baylou, P., and Germain, C. (2007). Estimating local multiple orientations. Signal Processing, 87(7):1655-1669.

Miller, K. L., Stagg, C. J., Douaud, G., Jbabdi, S., Smith, S. M., Behrens, T. E., Jenkinson, M., Chance, S. A., Esiri, M. M., and Voets, N. L. (2011). Diffusion imaging of whole, post-mortem human brains on a clinical MRI scanner. NeuroImage, 57(1):167-181.

Mori, S., Crain, B., Chacko, V., and van Zijl, P. (1999). Three-dimensional tracking of axonal projections in the brain by magnetic resonance imaging. Annals of Neurology, 45:265-269.

Mori, S. and Zhang, J. (2006). Principles of diffusion tensor imaging and its applications to basic neuroscience research. Neuron, 51(5):527-539.

Nieuwenhuys, R. (2013). The myeloarchitectonic studies on the human cerebral cortex of the Vogt-Vogt school, and their significance for the interpretation of functional neuroimaging data. Brain Structure and Function, 218(2):303-352.

Peters, A., Cifuentes, J., and Sethares, C. (1997). The organization of pyramidal cells in area 18 of the rhesus monkey. Cerebral Cortex, 7(5):405421.

Pullens, P., Roebroeck, A., and Goebel, R. (2010). Ground truth hardware phantoms for validation of diffusion-weighted MRI applications. Journal of Magnetic Resonance Imaging, 32:482-488.

Richardson, M. (2010). Current themes in neuroimaging of epilepsy: Brain networks, dynamic phenomena, and clinical relevance. Clinical Neurophysiology, 121:1153-1175.

Roebroeck, A., Galuske, R., Formisano, E., Chiry, O., Bratzke, H., Ronen, I., Kim, D., and Goebel, R. (2008). High-resolution diffusion tensor imaging 
and tractography of the human optic chiasm at 9.4 T. NeuroImage, 39:157168.

Sanides, F. (1962). Die architektonik des menschlichen stirnhirns. In Müller, M., Spatz, H., and Vogel, P., editors, Monographien aus dem Gesamtgebiete der Neurologie und Psychiatrie. Springer, Berlin.

Schmitt, O. and Birkholz, H. (2011). Improvement in cytoarchitectonic mapping by combining electrodynamic modeling with local orientation in high-resolution images of the cerebral cortex. Microscopy Research and Technique, 74(3):225-243.

Seehaus, A., Roebroeck, A., Bastiani, M., Fonseca, L., Bratzke, H., Lori, N., Vilanova, A., Goebel, R., and Galuske, R. (2015). Histological validation of high-resolution DTI in human post mortem tissue. Frontiers in neuroanatomy, 9.

Seehaus, A. K., Roebroeck, A., Chiry, O., Kim, D.-S., Ronen, I., Bratzke, H., Goebel, R., and Galuske, R. (2013). Histological validation of DW-MRI tractography in human postmortem tissue. Cerebral Cortex, 23(2):442450 .

Sporns, O., Tononi, G., and Kötter, R. (2005). The human connectome: a structural description of the human brain. PLoS computational biology, 1(4):e42.

Sundgren, P. C., Dong, Q., Gomez-Hassan, D., Mukherji, S. K., Maly, P., and Welsh, R. (2004). Diffusion tensor imaging of the brain: review of clinical applications. Neuroradiology, 46(5):339-350.

Tournier, J.-D., Calamante, F., and Connelly, A. (2007). Robust determination of the fibre orientation distribution in diffusion MRI: Nonnegativity constrained super-resolved spherical deconvolution. NeuroImage, 35(4):1459-1472.

Tournier, J.-D., Calamante, F., Gadian, D. G., and Connelly, A. (2004). Direct estimation of the fiber orientation density function from diffusion- 
weighted MRI data using spherical deconvolution. NeuroImage, 23(3):1176-1185.

Tuch, D. S. (2004). Q-ball imaging. Magnetic Resonance in Medicine, 52(6):1358-1372.

Vogt, C. and Vogt, O. (1919). Allgemeinere Ergebnisse unserer Hirnforschung. Journal für Psychologie und Neurologie, 25.

Wedeen, V., Wang, R., Schmahmann, J., Benner, T., Tseng, W., Dai, G., Pandya, D., Hagmann, P., D'Arceuil, H., and de Crespigny, A. (2008). Diffusion spectrum magnetic resonance imaging (DSI) tractography of crossing fibers. NeuroImage, 41(4):1267-1277.

Wedeen, V. J., Hagmann, P., Tseng, W.-Y. I., Reese, T. G., and Weisskoff, R. M. (2005). Mapping complex tissue architecture with diffusion spectrum magnetic resonance imaging. Magnetic Resonance in Medicine, 54(6):1377-1386. 
Chapter IV

Sources of orientation

decoding in $7 \mathrm{~T}$ fMRI data

In preparation 


\section{Abstract}

It has been reported in many cases that stimulus orientation can be decoded from human high-field fMRI data. However, it is under debate which functional patterns are learned by classification algorithms in this process. Proponents of the hyperacuity hypothesis claim columnar patterns to be the crucial source of information. Opponents of this view reject the idea of meaningful subvoxel information in favor of large-scale patterns. One possible pattern is the radial bias which is known to be observable in fMRI data. In this study, we replicate findings of a radial bias and orientation decodability in the visual cortex at high spatial resolution in a $7 \mathrm{~T}$ fMRI experiment. We critically assess reported methods to study the contribution of individual factors towards classification results and propose new alternatives. Applying these to test for the influence of radial bias on orientation decoding, we conclude that it likely plays a meaningful, but not pivotal role.

\section{Introduction}

Functional magnetic resonance imaging is maybe the most important imaging technology in modern neuroscience. It combines a reasonable spatial resolution with a temporal resolution high enough to conduct event-related experiments, while having only mild side-effects for the experimental subjects. Classically, in an fMRI experiment, stimuli of different conditions are presented to the subject via a screen or headphones, and activation time series of a set of voxels are acquired. In these data, it is tested whether the activation in response to one stimulus condition is significantly different to another condition. Most often, the time series are statistically compared in linear regression approaches such as the General Linear Model (GLM). These yield prediction weights for each condition, which in conjunction with condition contrasts result in t- or F-values for each voxel. The alpha-error corrected values can be visualized as heat maps, providing information about where in the brain the experimental 
conditions caused the highest activation differences. In this sense, fMRI experiments are univariate: every voxel represents a unique (even if not independent) statistical experiment.

In addition to this classical approach, multivariate designs subsumed under the label of multi-voxel pattern analysis (MVPA) have become very popular over the last 15 years (Haxby et al. 2001, Haynes and Rees 2006). Here, not individual voxels but the activation patterns of entire voxel populations are investigated. Mostly, such analyses are based on correlations of population responses towards different conditions (Kriegeskorte et al. 2008) or classifiers which are trained to distinguish the different activation patterns in a subset of the acquired data and then tested on a disjunct subset (Haynes and Rees 2005).

MVPA approaches are generally more sensitive than univariate approaches, being able to detect small condition differences across a voxel pattern which would not reach significance in individual voxels (Norman et al. 2006). On the other hand, the univariate approach is spatially more specific. Where an MVPA only provides information about response differences in an entire voxel pattern, a GLM shows precisely where these differences are most pronounced - given that they are sufficiently strong on the level of individual voxels. For this reason, MVPA studies are commonly conducted in conjunction with localizer experiments, allowing for an hypothesis-driven a priori selection of voxel populations.

Besides trading spatial information against statistical power, there are other notable features of MVPA. In the case of correlational analyses, MVPA enables structural comparisons between very different data sources. For example, one could present stimuli of different object classes to a human in an fMRI experiment and to a neuronal model and then compare the mutual class correlations for both the fMRI data and the model output (Connolly et al. 2012). While this possibility is very helpful, the results have to be interpreted cautiously because the different data sources are brought together on a very small common denominator. In the example, a shared correlation pattern would be seen as evidence for the biological 
plausibility of the model, but it would be rather weak evidence since also very different computational structures can result in similar correlation patterns.

In the case of classifier analyses, MVPA allows to decode cognitive content from measured data. This is especially appealing when (after an initial training phase) the decoding can be performed in real-time, providing the basis for brain-computer interfaces $(\mathrm{BCI})$. Even though the practical feasibility of BCIs is still limited, there has been considerable progress over the last years (Sorger et al. 2009). This is certainly due to the vast potential applicability of BCIs, ranging from clinical scenarios (communicating with locked-in patients) to everyday tasks (operating machines via cognitive commands).

In fundamental research, another reason to do classifier studies is the hope to find information about the "coding pattern" in the brain which underlies the observed voxel pattern. This can be attempted in various ways, for example by providing stimuli that are matched for certain features, by manipulating the acquired data and testing the effect on decoding performance, or by modeling voxel patterns and comparing the decoding performance to the empirical findings. This approach has most notably been adopted in the research of visual perception, aiming to contribute towards the controversial debate about functional orientation columns in the human visual cortex (Horton and Adams 2005, Rockland 2010).

Here particularly, it turned out that the interpretation of classification results itself is highly controversial. It has been found in many studies that seen orientations can be decoded from evoked brain patterns in the visual cortex with high accuracy already at voxel sizes of $3 \mathrm{~mm}$. Yet there has been a continuing debate about the main source of information leading to this capability. At present, there are two predominant explanations for the decodability of orientations: The hyperacuity hypothesis states that classifiers such as support vector machines (SVM) are able to pick up the tuned responses of orientation columns well below the actual 
voxel resolution (Kamitani and Tong 2005). This is due to the fact that these columns are not homogeneously distributed with respect to the imaging voxels such that each voxel exhibits a small orientation bias in a random but stable fashion. As a result, the voxel population has a distinct orientation preference pattern at a high spatial frequency. Contrarily to that, one could suspect orientation biases on a larger spatial scale to be responsible for the decoding performance (Op de Beeck 2010, Freeman et al. 2013). One prominent candidate of such a global bias is the radial bias, which describes the perceptual tuning towards orientated stimuli that lie radially in the visual field, pointing towards its center. This bias has repeatedly been shown in both behavioral and imaging experiments (Sasaki et al. 2006, Sun et al. 2012). Lastly, there are attempts to explain stimulus decodability without either local or global biases. Carlson (2014) for example decodes stimuli from cell patterns of an unbiased V1 model using the distinct superposition patterns between model cells and the stimulus borders.

Most of the early influential fMRI classifier studies have been conducted at $3 \mathrm{~T}$ systems. Over the past decade, ultra-high-field scanners have become available for fMRI studies in humans. This made remarkably finer spatial resolutions in the order of around $1 \mathrm{~mm}$ possible, allowing for example to identify columnar patterns for different modalities in fMRI maps (Yacoub et al. 2007, 2008, Zimmermann et al. 2011). In the context of the debate outlined above, this increase in resolution could prove helpful as well. Particularly, one of the arguments in favor of hyperacuity was a scattered pattern of orientation preference observed at $3 \mathrm{~mm}$ voxel size. It would provide a valuable, although indirect, piece of information to see whether maps of features relevant in the classification process also show a scattered pattern at small voxel sizes.

The aim of this study therefore was to continue previous research attempts with better data quality, but also to conceptually assess some of the arguments brought forward in past studies. To this end, we conducted a $7 \mathrm{~T}$ fMRI experiment where subjects viewed different types of oriented stimuli. Our research questions were: 
1. Does the orientation preference of voxels in the visual cortex exhibit a radial bias?

2. Can the observed orientations be decoded using the entire voxel population or even using only parts of the visual field?

3. If so, does the classification performance show a radial bias?

4. How likely are hyperacuity, radial bias, or stimulus borders to account for decoding results?

One particular argument which we addressed in some depth is the refutation of the radial bias as a contributor to orientation classification given in Kamitani and Tong (2005). The authors report a control experiment where they divide the visual field into 16 wedges and in each of those wedges z-transform the activation pattern for each trial. They find the classification performance unaffected by this transformation and conclude that radial bias could not be involved in the classification process. This argumentation, despite sounding logical at first, is flawed: The problem is that the applied normalization does not remove an existing radial bias pattern within the visual field segments (Misaki et al. 2010). Instead, the pattern only becomes discontinuous at the arbitrarily chosen segment borders. To completely hide a source of information from the classifier, as was attempted here, one would need to know the exact functional relation between this source and the data and would have to apply a voxel-based correction. Notably, this correction would have to be precise enough that the remaining information in the residuals would not be sufficient to get picked up by the classifier. Hence the only possibly adverse effect of the applied transformation for a classifier relying on radial bias information is that the normalization decreases the signal-to-noise ratio (SNR) of the response pattern. This is because in the case of a radial bias the signal range is larger in the entire pattern than within segments. To demonstrate this, we calculated a simulation with an idealized radially biased voxel population.

If these considerations are correct, then the conclusions that can be drawn from this kind of control experiment are: First, if the normalization 
does not affect the performance, then either the global pattern was irrelevant or the classifier can still sufficiently perform at the lower SNR caused by the data binning. Second, if the normalization impairs the performance, then a global pattern was indeed involved and the normalization decreased the SNR in a significant fashion.

As a consequence, a noneffective normalization is relatively inconclusive. A possibility to proceed in this case is to increase the number of bins to make it more likely that if a global bias is involved, the SNR is lowered enough to make the normalization effectful. However, classifiers, particularly support vector machines (SVM), have been reported to be very robust to data noise (Op de Beeck 2010), so this attempt could prove difficult in practice. Also, it comes with a possible confound: Since voxel data are discrete, making their pattern discontinuous comes at the cost of the information at the bin borders. In the extreme case, if each voxel is an individual bin, all the information in the data is lost (everything is normalized to 0 ) and the classification has to fail. To control for this source of information loss, one could apply two different types of binning: One with a pattern in line with the assumed global bias, and another with an orthogonal pattern. In the case of the radial bias, this means one should divide the visual field into wedges and annuli. While the wedge normalization reduces the SNR of a radially biased voxel pattern, the annulus normalization should not affect it at all. Therefore, if the performance breaks down for both kinds of normalization at the same time, then it is probably caused by the mere data reduction. If on the contrary, the performance on wedge-transformed data decreases faster, a radial bias was probably involved.

An alternative to this extended normalization approach is to perform the classification on individual data bins right away and to average the resulting performance values. In this case, it is guaranteed that only a fraction of the entire global pattern is available to the classifier, so this should be more likely to have an impact if the pattern in question is involved in the classification process. Here, a confounding factor is the 
reduction of the feature space, so the results should be compared to voxel subsets randomly drawn from the entire population.

We report results of both outlined procedures to test for an involvement of the radial bias in orientation decoding.

\section{Materials and Methods}

\subsection{Subjects}

Three subjects ( 1 female, 2 male) of age 27, 33 and 27 participated in the study. All subjects were healthy and had normal or corrected to normal vision.

\subsection{Stimuli}

All visual stimuli were created and presented using custom written presentation software, which is freely available on the web (https:/ /github.com/ svengijsen/BrainStim). The stimuli were projected onto a screen in the rear of the magnet and presented to the subjects via mirrors. The stimulation screen had a size of $(30 \times 18) \mathrm{cm}$ and was located at a distance of $99 \mathrm{~cm}$ to the subjects' eyes, resulting in a visual field coverage of $(17.2 \times 10.4)^{\circ}$ visual angle. The grating and the flow field part of the experiment used the full screen for stimulus presentation whereas the pRF stimuli were presented on a square screen, leaving the left and right sides of the screen gray.

We conducted a pRF mapping using the parameters described in Senden et al. (2014): The stimuli were bars of flickering checkerboard, presented at four different orientations $\left(0^{\circ}, 45^{\circ}, 90^{\circ}, 135^{\circ}\right)$ and twelve different positions per orientation. The width of the bars was 1/12-th of the screen size such that all twelve positions together covered the whole presentation area. One pRF run consisted of two times eight randomly ordered orientation trials (two per orientation), in which a bar of the respective orientation was presented once at each position (again in random 
order). The bars remained in one position for the duration of one TR, which was $2 \mathrm{~s}$. In total, this amounted to $4 * 8 * 12 * 2 \mathrm{~s}=12.8 \mathrm{~min}$ run duration.

In the main experiment, square-wave gratings were presented with a spatial frequency of 1.5 cycles per degree and one of eight different orientations $\left(0^{\circ}, 22.5^{\circ}, \ldots, 157.5^{\circ}\right)$. To evoke neuron responses independently of their spatial phase tuning, the gratings flickered between random positions at a frequency of $2 \mathrm{~Hz}$. To make the stimuli more strainlessly watchable over the course of the experiment, a screen contrast of $53 \%$ was chosen (60/195 gray level), which feels more pleasant to the eye than a $100 \%$ contrast while leaving the grating easily recognizable. The experimental structure was as follows: The basic presentation unit was a trial which was the presentation of the grating at one particular orientation for $16 \mathrm{~s}$, followed by a $6 \mathrm{~s}$ break (gray screen). A run consisted of four series à eight trials (one of each orientation). The trials were ordered such that run 1 and 2 together formed a balanced latin square, i.e. each orientation preceded and succeeded each other orientation the same number of times. Each subject performed four runs (with runs 3 and 4 identical to 1 and 2), with a total duration of $49.1 \mathrm{~min}$ for the main experiment.

Finally, the stimuli in the flow field experiment were patterns of dots moving according to four different experimental conditions: Straight motion away from the center, rotation around the center, random motion and no motion. Here, a trial was the presentation of one condition for $16 \mathrm{~s}$, followed by $2 \mathrm{~s}$ break. Each run consisted of four series à four trials (one per condition) such that between every expansion and rotation trial, there was one of the two control conditions. In the rotation condition, clockwise and counterclockwise motion direction were evenly matched. Each subject performed three of these runs, with a total duration of $15.9 \mathrm{~min}$. While the main experiment allowed for an assessment of radially biased voxel responses for each individual orientation, this experiment was supposed to address the radial bias globally (expansion versus rotation), with random and static motion as control conditions. Thus, this experiment 
was expected to yield less detailed but more robust results than the grating part.

\subsection{MRI acquisition}

The MRI measurements were conducted with a 7T whole-body MRI system (Magnetom, Siemens, Erlangen, Germany) located at the Maastricht Brain Imaging Center (Maastricht, The Netherlands). The RF coil used was a single channel transmit, 32 channel receive head-coil (NOVA medical, Wilmington MA, USA).

For anatomical imaging, a MP2RAGE sequence with the following parameters was employed: $\mathrm{TR}=5000 \mathrm{~ms}, \mathrm{TE}=2.47 \mathrm{~ms}$, TIs $=$ 900 and $2750 \mathrm{~ms}$, GRAPPA acceleration factor $=3$, nominal flip angles = $3^{\circ}$ and $5^{\circ}$, matrix size $320 \times 320 \times 240$ at a nominal voxel size of $0.7 \mathrm{~mm}$ isotropic, total acquisition time $=8.03 \mathrm{~min}$. The slices were chosen in oblique transversal position such that the field of view covered the entire occipital lobe as well as a parts of the parietal and temporal lobes.

Functional images were acquired using a multi-band EPI sequence (Moeller et al. 2010) with the parameters: TR $=2000 \mathrm{~ms}$, TE $=19 \mathrm{~ms}$, multi-band acceleration factor $=2$, GRAPPA acceleration factor $=2$, nominal flip angle $=60^{\circ}$, matrix size $=128 \times 128 \times 70$ at a nominal voxel size of $1.25 \mathrm{~mm}$ isotropic, echo spacing $=0.75 \mathrm{~ms}$. The transmit voltage was adapted based on a $B_{1}$ mapping such that the achieved flip angles matched the nominal values in a ROI in early visual cortex. To correct for EPI distortions in the preprocessing step, two short reference runs with opposite phase encoding direction were acquired for each subject.

\subsection{Analysis}

All analyses were conducted in BrainVoyager QX (v3.0, Brain Innovation, Maastricht, The Netherlands) or Matlab (v7.14, MathWorks, Natick MA, USA) along with the NeuroElf toolbox (Jochen Weber, Columbia University). 


\section{Data preprocessing}

The anatomical data were first resampled at a voxel size of $0.625 \mathrm{~mm}$ isotropic (half the functional voxel dimension). Then, inhomogeneity correction, brain extraction and transformation to ACPC space were conducted. Last, inflated surface meshes for both hemispheres were created.

On the functional data, slice scan time correction and rigid body (three translations, three rotations) motion correction were performed. The average translation and rotation parameters for the subjects were $\left(0.44 \mathrm{~mm}, 0.54^{\circ}\right),\left(2.20 \mathrm{~mm}, 1.65^{\circ}\right)$ and $\left(1.23 \mathrm{~mm}, 0.65^{\circ}\right)$. Next, EPI distortions were corrected for, using BrainVoyager's Cope plugin (Fritz et al. 2014), which works similar to the Topup algorithm in FSL (Andersson et al. 2003). Last, the data were temporally high-pass filtered with a threshold of 2 cycles per run to exclude slow drifts in the data.

\section{Receptive field mapping}

In the population receptive field paradigm, each voxel is assigned a receptive field, which is a two-dimensional Gaussian within the stimulated part of the visual field. It is determined by its center in polar coordinates (eccentricity, polar angle) and standard deviation (receptive field size). Whenever the flickering bar in the pRF run falls within a voxel's receptive field, the voxel is expected to respond, and the model parameters are calculated such that this prediction matches the measured time series optimally. The calculations were performed using the functionality implemented in BrainVoyager which follows the procedure described in Dumoulin and Wandell (2008). Using the pRF data, the whole voxel set was divided into eight subpopulations, coding for each of eight pairs of opposite wedges, corresponding to the orientation axes of the stimuli. This division allowed to test hypotheses on a radial bias in the data which would cause stimuli to raise the highest activity in those visual field sectors where their orientation was radial. Since most centrally in the visual field, the receptive field center of a voxel can arbitrarily fall into any of the wedge-pairs, only 
voxels with an eccentricity of at least $1^{\circ}$ were selected. The retinotopy maps provided by the pRF data were also used to localize visual areas V1 to V4, the region of interest for all further analyses.

\section{Gratings}

The data of the main experiment were analyzed by means of fitting them to a general linear model (GLM). This is a multiple linear regression of each voxel's time series where each experimental condition is convolved with an idealized hemodynamic response function to obtain a predictor (Goebel 2016). In matrix notation, this equates to

$$
y=X b+e
$$

where $y$ is the time series of a voxel. $X$ is the design matrix, its columns are the predictors that are obtained by convolution of the characteristic function of the stimulus presentation time (1 where stimulus of the respective condition was shown, 0 otherwise) with the hemodynamic response function. The vector $b$ of beta values is determined by minimization of the squared error term $e$ through the formula

$$
b=\left(X^{T} X\right)^{-1} X^{T} y
$$

Thus, the GLM yields for each voxel a vector of one beta value per condition. The beta values are z-scores which indicate the relative importance of each condition for explaining the voxel's time course. Two kinds of GLM were fitted to the data: One where the entirety of trials for each orientation together formed a predictor, one where each trial was an individual predictor.

The first, condition-based GLM served to determine voxel response profiles towards the different orientations, and to check whether these are radially biased. The response profiles were acquired in the form of $\mathrm{t}$-values for orientation-specific contrasts (one orientation vs rest). They were derived from the beta vectors following the formula

$$
t=\frac{c^{T} b}{\sqrt{\operatorname{Var}(e) c^{T}\left(X^{T} X\right)^{-1} c}}
$$


Here, $c$ is the contrast vector, for example $(7,-1, \ldots,-1)$. Subsequently, the $t$-values were averaged over section populations.

Our hypothesis was that for all visual field segments, the largest average $t$-values would be found at radial orientations. This interaction effect between receptive field position and orientation preference could potentially be overshadowed by large main effects of orientation preference, such as a strong oblique effect (Furmanski and Engel 2000). To rule this possibility out, we z-transformed the $\mathrm{t}$-values within orientation conditions. That is, for each stimulus orientation, they were normalized by their means and standard deviations $\left(\frac{x-\mu}{\sigma}\right)$ across the whole voxel population.

The beta values of the second, trial-based GLM were the basis for the classifier analysis. We classified orientations separately in each of the eight visual field segments, in the entire voxel population and in a random subset of one eigth of the voxel population. Each of the eight orientation conditions was presented four times in each of the four runs, resulting in 128 beta values per voxel. In a leave-one-run-out design, we trained a support vector machine to discriminate voxel patterns of each of the eight orientations. While SVMs are principally binary classifiers (they determine a hyperplane in the feature space to optimally divide two sets of data points), multiple SVMs can be combined to solve $n$-ary classification problems like the one at hand. One way to do this is the 1-vs-1-approach where the classifier is trained and tested separately on all pairs of conditions (Hsu and Lin 2002). In this case, the performance for one particular orientation is simply the sum of correct divided by the sum of wrong classifications of this orientation versus any of the other orientations. As a result, we obtained in each of the eight visual field segments percentages of correct classification for each of the eight orientations, with a chance level of $50 \%$.

In the training process, an SVM selects a subset of training trials as support vectors and assigns weights to them. The sign of the weighted average scalar product between the support vectors and a test vector (plus some intercept) determines its assigned class. To visually explore which 
kind of information gets learned from our data, we obtained weighted averages of the support vectors for selected binary classifications and displayed them on the cortical surface (Fig. 2c).

We also tested the possibility that classification performance crucially depends on the information of the stimulus borders. To this end, we compared the classification performance in those voxels whose receptive fields did not reach the borders of the stimulus screen (Eccentricity $+2 *$ RF size $\leq 5.2^{\circ}$ ) to the performance in a random equally sized subset of all voxels.

\section{Effect of radial normalization}

Similarly as reported in Kamitani and Tong (2005), the trial-based beta values were normalized within trials and eight visual field double-wedges. Additionally to this polar angle binning, the normalization was conducted for an eccentricity-based binning (i.e. parcellation of visual field into annuli) and with bin counts of 16, 32, 64, 128 and 256. A subdivision into more bins was not feasible due to the limited accuracy of the localizer data and the pRF model. The same classification procedure as described above was applied to the normalized data sets.

We also simulated a population of 800 radially biased voxels with receptive field centers evenly distributed between 0 and $2 \pi$ (polar angle; eccentricity was not considered in the simulation). The voxel response towards a stimulus of orientation $\theta$ as a function of its receptive field center was a Gaussian $(\mu=\theta, \sigma=\pi / 3)$ cyclically wrapped around the interval $[0,2 \pi]$ plus random Gaussian noise $(\mu=0, \sigma=0.3)$. As in the main experiment, population responses towards 16 trials of each of eight orientations were created. Then, the voxel population was divided into eight equally sized polar angle bins and voxel activities within these bins were normalized for each trial. With the same design as in the main experiment, the different orientations were classified from the untransformed and the transformed data. 


\section{Flow fields}

We determined $t$-values for each of the six pairs of different experimental conditions in the same fashion as in the main experiment. Our main focus was on the contrast of expanding versus rotating dots, with the random motion and the static dots being control conditions. Since this contrast was meant to reflect radial preference throughout the whole visual field, the voxel population was not divided into circle segments here but into a foveal $\left(<2^{\circ}\right.$ visual angle) and a parafoveal $\left(2-5.2^{\circ}\right)$ subpopulation. By hypothesis, the expansion condition should yield a larger response than the rotation condition in the parafoveal, but not so much in the foveal part of the visual field.

\section{Results}

\subsection{Orientation preference shows radial bias}

The orientation preference profile examined individually for the different visual field segments revealed a strong radial bias in all three subjects (Fig. 1). The finding of a radial bias was visually confirmed comparing patterns of receptive field centers, $\mathrm{t}$-values and SVM support vectors displayed on the cortical surface (Fig. 2). The z-transformation we applied had an effect since the untransformed orientation profiles showed cardinal biases in two of the three subjects (particularly strong in subject 2, Fig. 3).

\subsection{Orientation can be robustly decoded within visual field wedges}

The classification performance the subjects achieved in each of the visual field segments is shown in Figure 4. The average performance values over the eight visual field segments were $.9262, .7196$ and .8767 for the three different subjects. The classification performance reached based on the full voxel population was remarkably higher at $.9710, .8036$ and 

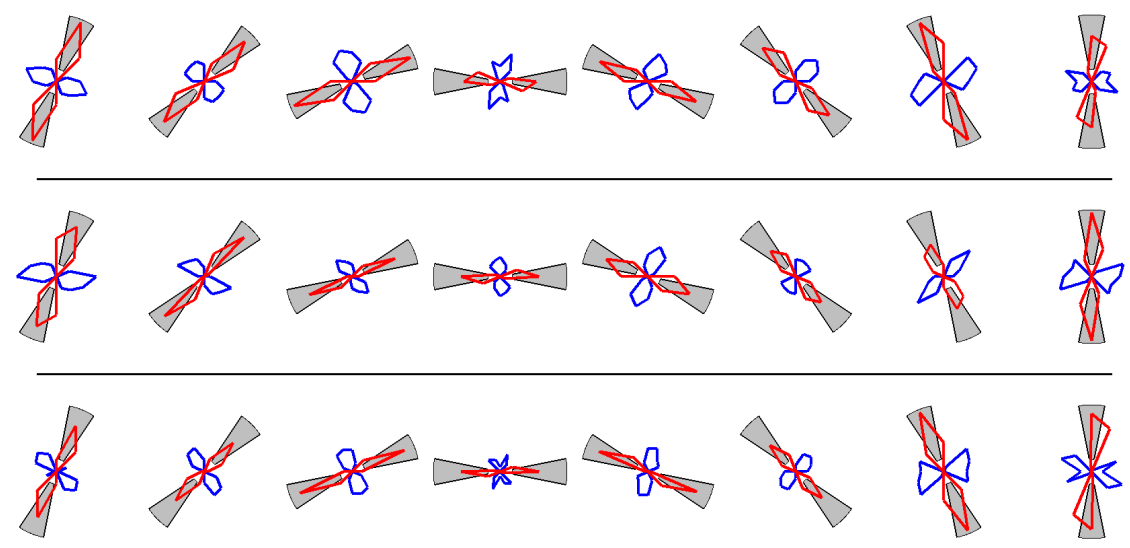

Figure 1: Polar plots of average t-values across visual field segments. The value at an angle $\alpha$ shows the $t$-value obtained for the contrast of orientation $\alpha$ versus rest. Red indicates positive, blue negative $t$-values. Columns correspond to visual field segments as displayed in gray. Rows correspond to different subjects.

.9330, respectively. In the random population subset, the classification performance was marginally reduced compared to the full population in two out of three subjects $(.9643, .7612, .9342)$.

\subsection{Radial bias is strong for orientation preference, weak for classification performance}

In order to obtain a measure of the amount of radial bias in the above results, we averaged data sharing the same relation between visual field segment and presented stimulus orientation. That is, we obtained average $\mathrm{t}$-values and classification performance rates for orientations which are totally radial with respect to the visual field segment, which are one angle step (22.5 ) apart, two steps, and so forth (Fig. 5). For the orientation preference profiles, this again showed the strong radial bias in all three subjects. In the classification performance profiles, a radial bias was only observable for subject 3 and was much less pronounced than the radial biases of the orientation preferences. 

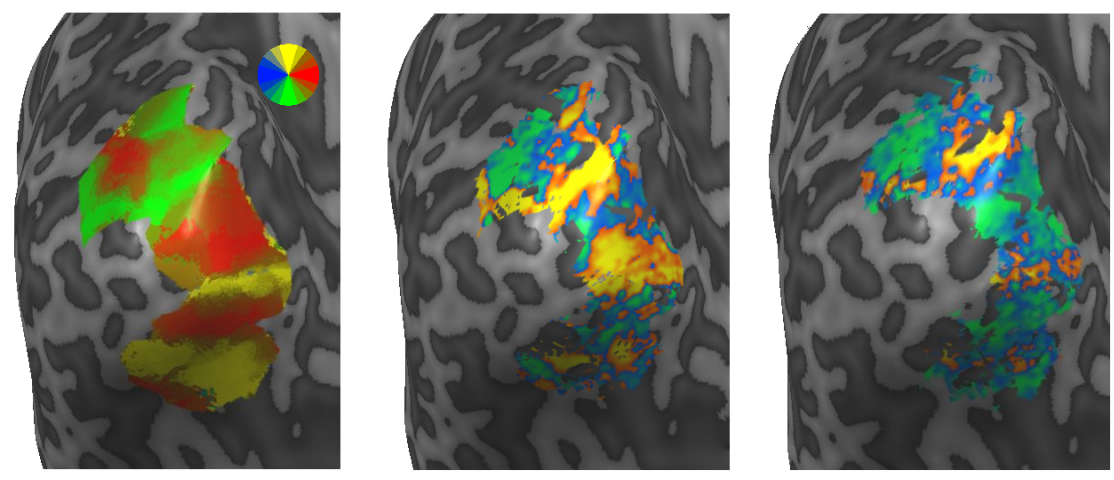

Figure 2: Surface maps of left visual cortex of subject 1. a: Polar angles of voxel receptive field centers. b: z-transformed t-values for $90^{\circ}$ vs rest. c: Weighted SVM support vectors for the $0^{\circ}$ vs $90^{\circ}$ classification. All maps are strongly spatially correlated.

\subsection{Border information is not necessary for orientation decod- ing}

The performance in the inner part of the stimulus screen was almost identical to the performance over the whole stimulus area (Subject 1: 9654 vs .9643 , Subject 2: .8013 vs .8013 , Subject 3: .9375 vs .9420 ).

\subsection{Data normalization has no effect on classification perfor- mance}

Neither the data normalization within wedges nor within annuli had a remarkable effect on the classification performance, even for high bin counts (changes in average performance $<1 \%$ in all subjects).

The simulation visually demonstrates our reasoning that the normalization only decreases the SNR but does not remove the global pattern (Fig. 6). 

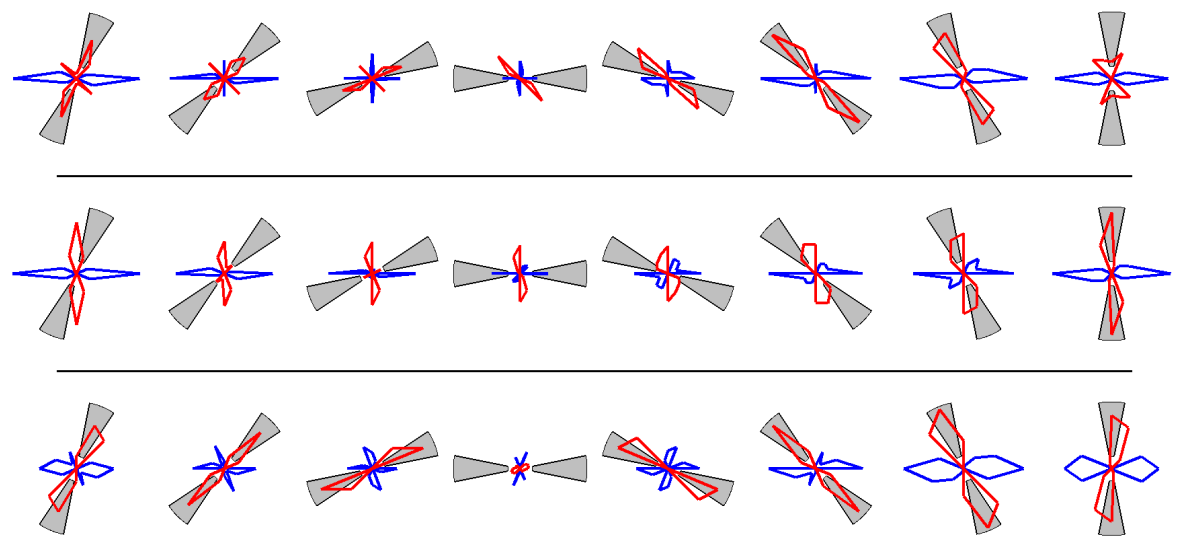

Figure 3: Polar plots of average t-values as in Figure 1, but without $\mathrm{z}$-transformation of t-values. Global biases towards horizontal and, in subject 2, vertical orientation are apparent.

\subsection{Flow field stimulation evokes slightly radially biased re- sponse with inverse sign}

The main results of the flow field analysis are shown in Table 1. In all subjects, the rotation condition caused a greater or equal voxel response than the expansion condition, although this effect reached significance only in one subject. The absolute $t$-values for this contrast were remarkably larger in the parafoveal than in the foveal voxels, although for subject 2 both t-values were small. In most cases, both the rotation and the expansion condition activated voxels more strongly than the static control condition. With the exception of subject 2, this effect was again largest parafoveally, especially for the rotation versus static contrast.

\section{Discussion}

The voxel responses we acquired towards different oriented stimuli showed a clear radial bias for all three subjects. In the main experiment, this was shown through orientation preference profiles obtained as t-values for different orientation contrasts. In the flow field experiment, it was 


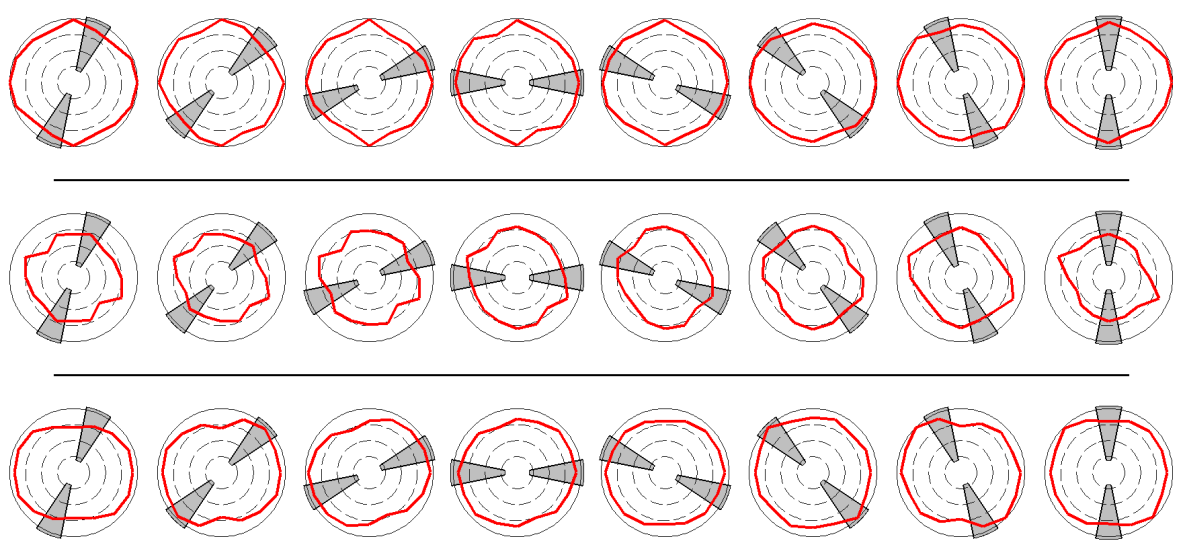

Figure 4: SVM classification performance for different stimulus orientations across visual field sectors. Outer circle indicates $100 \%$ performance. Columns correspond to visual field segments as displayed in gray. Rows correspond to different subjects.

Table 1: Average t-values $(d f=311)$ for foveal and parafoveal ROI in the flow field experiment. ${ }^{*}$ indicates significance at $\alpha<.05$ level

\begin{tabular}{ccccccc}
\hline & \multicolumn{2}{c}{ S01 } & \multicolumn{2}{c}{ S02 } & \multicolumn{2}{c}{ S03 } \\
\hline & Fov. & Paraf. & Fov. & Paraf. & Fov. & Paraf. \\
Exp. - Rot. & $-0,89$ & $-1,67$ & 0,04 & $-0,01$ & $-0,97$ & $-2,75^{*}$ \\
Exp. - Stat. & 0,49 & 0,99 & 0,88 & 0,70 & 0,18 & $-0,48$ \\
Rot. - Stat. & 1,39 & $2,67^{*}$ & 0,85 & 0,71 & 1,16 & $2,29^{*}$ \\
\hline
\end{tabular}

indicated by the activation difference between expanding (radial) and rotating (tangential) dot patterns which was significant in the parafoveal visual field part of one subject and marginally significant for another one. Contrary to our assumptions however, the radially moving dots were associated with a significantly lower activity compared to the tangentially moving dots, so these findings have to be interpreted cautiously. The flow field experiment was an alternative way of testing for a radial bias which was thought to be more robust due to its global nature (no individual testing for several visual field parts necessary). Since the main experiment was clearly sensitive enough to demonstrate the radial bias, the flow field 

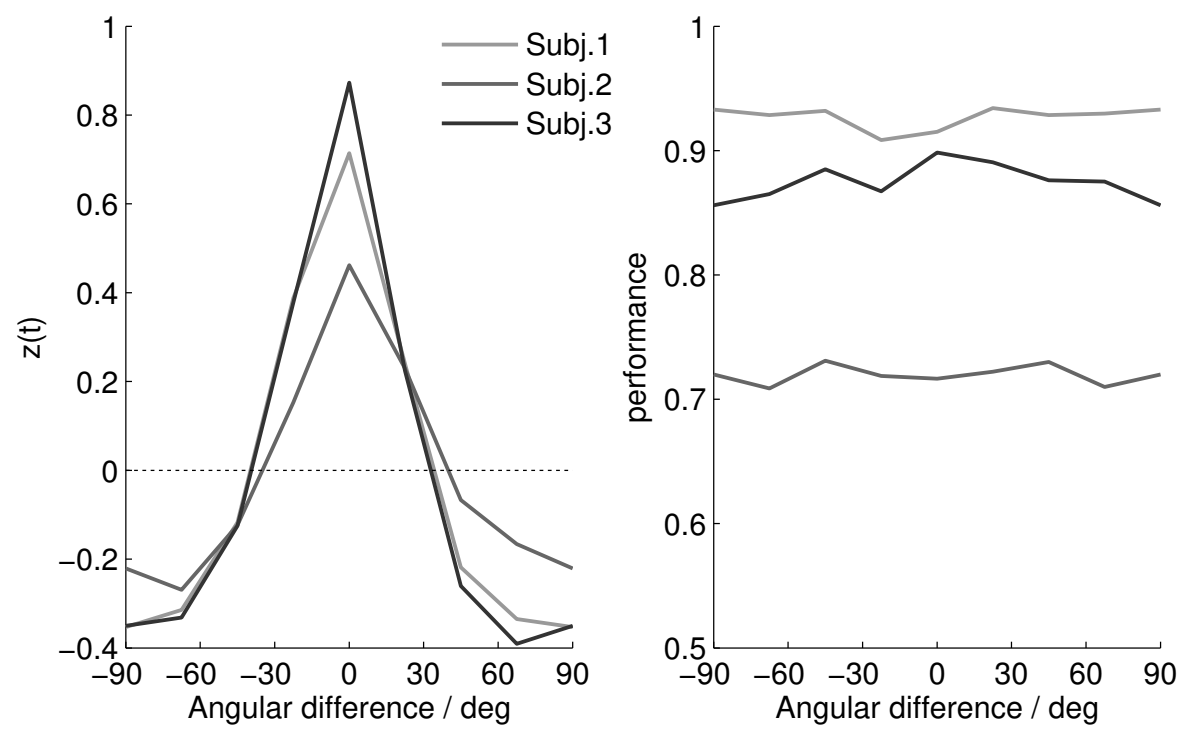

Figure 5: "Radiality" of average t-values (left) and classification performance (right) over the entire (stimulated) visual field. The value at $x$ represents the average of all data points for pairs of orientation contrast and visual field segment whose angle is different by $x$.

part does not contribute any additional information to our main findings and will therefore be omitted in future studies.

Using support vector machines, it was possible in our data to classify each of the eight presented grating orientations against the others. The performance was well above chance level in all subjects, in two out of three it was greater than $90 \%$. This classification was also possible within visual field wedges at moderately reduced performance, a result which so far has not been reported elsewhere. The fact that classification was possible within visual field wedges, where only one section of a radial bias pattern can be present, indicates that this bias is probably not a crucial factor for the SVM. However, the performance within the wedges was more strongly reduced compared to the whole voxel population than in a random voxel subset, which gives evidence for at least a mild influence. This interpretation is supported by the visual inspection of averaged SVM support vectors, revealing a strong spatial correlation between these 

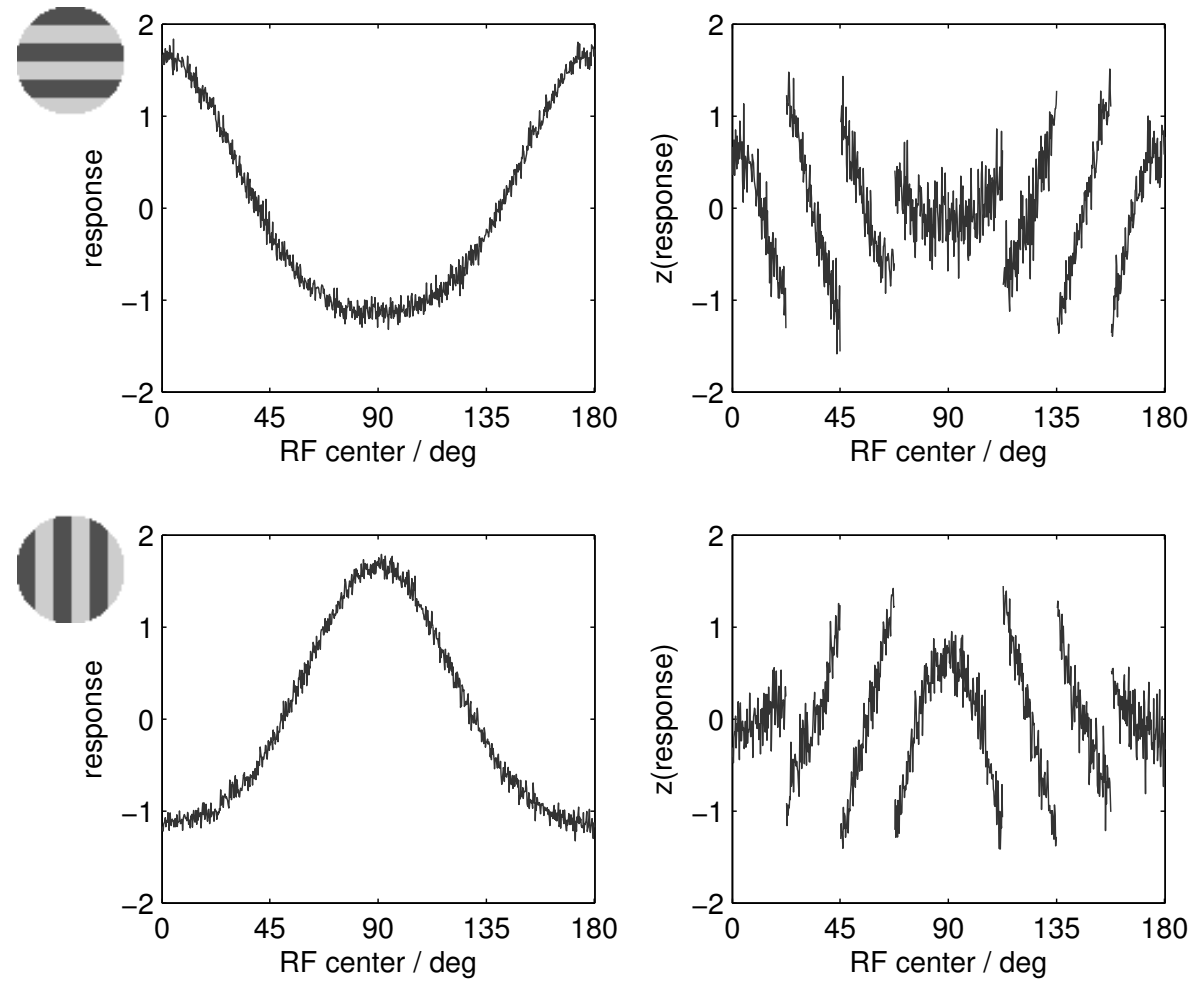

Figure 6: Simulated effect of z-transformation on voxel patterns. Plotted are the responses of 800 simulated radially biased voxels ordered along the $\mathrm{x}$-axis by the polar angle of their receptive field center (ranging linearly from 0 to $2 \pi$ ). The top row shows responses towards a $0^{\circ}$, the bottom row towards a $90^{\circ}$ stimulus, each averaged over 16 trials. On the left side, responses are untransformed, on the right side z-transformed within visual field segments and trials. Both pattern pairs can easily be discriminated (perfect SVM performance), although the normalized data are noisier. 
voxel patterns, the orientation preferences and the polar angles of the receptive field centers. These results are in line with a more recent study where already at the data acquisition stage, subjects were presented with stimuli matched for radial information. The matched stimuli could still be decoded but at a decreased performance (Alink et al. 2013).

The conclusion that the radial bias is one contributor to orientation decoding among others seems to contradict an earlier study claiming that the radial bias was controlled for (Kamitani and Tong 2005). However, we demonstrated in a simulation that the performed control measures were ineffective: A data normalization within visual field wedges can only negatively affect the classification if the radial bias contributes to the performance and the normalization changes the noise level precisely from a manageable to a disruptive level. We proposed an improvement of this kind of control experiment where data are normalized with respect to two different orthogonal binnings and series of increasing bin counts. In this case, the performance has to decline eventually and the information whether it decreases faster in one of the binning types could potentially give evidence for or against a certain influential global pattern. However, in our study, the resolution of the pRF model was not sufficient to test bin counts high enough to impair the SVM performance. At 256 bins, it was still unaffected. This gives evidence for the invariance of SVM classification to data normalization, as reported in earlier studies (Misaki et al. 2010). We can conclude that even when conducted in an elaborate fashion, data normalization is not a suitable way to investigate the informational basis of MVPA results. Instead, classification should be performed independently on subsets of the voxel pattern, as was done here with the visual field wedges.

If global biases provide only a small part of the information needed to successfully decode stimulus orientation, then the question remains on what spatial scale the major part of the information lies. Possibilities are either a subvoxel scale, as claimed by the hyperacuity hypothesis, or a mesoscale in the order of some millimeters/voxels. Our study does not allow to directly test for this. However, a visual inspection of SVM 
support vectors on the cortical surface revealed mesoscale patterns within the global, radially biased, organization. A scattered organization on the contrary, which could be expected in the case of hyperacuity, was not observed. There have been many arguments in past studies against hyperacuity, such as the robustness of SVM results against spatial smoothing, downsampling and subject motion, even between sessions. Taken together, we find the assumption of a mesoscale cortical pattern of orientation representation much more compelling than a subvoxel organization, at least in terms of orientation classification. Future studies should therefore form hypotheses about possible types of organizational patterns in the millimeter range and develop ways to test for those.

Addressing the simulation study by Carlson (2014), we classified stimulus orientation on a voxel population without access to stimulus borders. We found the same performance as in a random voxel subpopulation of the same size, which was almost as good as in the entire voxel population. This does not prove that voxel responses towards the stimulus borders are irrelevant, since we have a ceiling effect in our performance values making them even more robust to the loss of some information. But it shows that border information is not necessary for decoding performance and we can conclude that if stimulus edges are a contributor to overall classification performance, they are, just as the global biases, only one amongst several.

In summary, we can note that it is rather easy to find possible contributing factors for the decoding of fMRI data, since every systematical pattern can possibly be picked up by a classifier. However, it is hard to judge which information is actually used and which is not. Even if an organizational pattern is clearly present in a data set, such as the radial bias in our study, it can play only a minor role as compared to other effects which stay hidden to the applied line of analysis. So far, the most reliable source of evidence is if one manages to impair the classification performance by removing a certain source of information. The logic behind this is the same as in the findings made with brain-lesioned patients: If removal of some structure negatively affects function, then the structure was necessary. This however, especially with large data sets, is often difficult to achieve due to 
the superb robustness of classifiers. Other approaches such as showing the noninfluence of data transformation steps, classifying simulated data etc. can provide useful indications as well, but no hard evidence due to their indirect nature.

\section{References}

Alink, A., Krugliak, A., Walther, A., and Kriegeskorte, N. (2013). fMRI orientation decoding in V1 does not require global maps or globally coherent orientation stimuli. Frontiers in psychology, 4.

Andersson, J. L., Skare, S., and Ashburner, J. (2003). How to correct susceptibility distortions in spin-echo echo-planar images: application to diffusion tensor imaging. NeuroImage, 20(2):870-888.

Carlson, T. A. (2014). Orientation decoding in human visual cortex: new insights from an unbiased perspective. The Journal of Neuroscience, 34(24):8373-8383.

Connolly, A. C., Guntupalli, J. S., Gors, J., Hanke, M., Halchenko, Y. O., Wu, Y.-C., Abdi, H., and Haxby, J. V. (2012). The representation of biological classes in the human brain. The Journal of Neuroscience, 32(8):2608-2618.

Dumoulin, S. O. and Wandell, B. A. (2008). Population receptive field estimates in human visual cortex. Neurolmage, 39(2):647-660.

Freeman, J., Heeger, D. J., and Merriam, E. P. (2013). Coarse-scale biases for spirals and orientation in human visual cortex. The Journal of Neuroscience, 33(50):19695-19703.

Fritz, L., Mulders, J., Bremann, H., Peters, J., Bastiani, M., Roebroeck, A., Andersson, J., Ashburner, J., Weiskopf, N., and Goebel, R. (2014). Comparison of EPI distortion correction methods at 3T and 7T. In OHBM 2014 Annual Meeting, Hamburg, Germany. poster 1707.

Furmanski, C. S. and Engel, S. A. (2000). An oblique effect in human primary visual cortex. Nature neuroscience, 3(6):535-536. 
Goebel, R. (2016). BrainVoyager user guide: The general linear model (GLM). http://support.brainvoyager.com/functional-analysisstatistics /35-glm-modelling-a-single-study/82-users-guide-thegeneral-linear-model.html. [accessed 03/10/2016].

Haxby, J. V., Gobbini, M. I., Furey, M. L., Ishai, A., Schouten, J. L., and Pietrini, P. (2001). Distributed and overlapping representations of faces and objects in ventral temporal cortex. Science, 293(5539):2425-2430.

Haynes, J.-D. and Rees, G. (2005). Predicting the orientation of invisible stimuli from activity in human primary visual cortex. Nature neuroscience, 8(5):686-691.

Haynes, J.-D. and Rees, G. (2006). Decoding mental states from brain activity in humans. Nature Reviews Neuroscience, 7(7):523-534.

Horton, J. C. and Adams, D. L. (2005). The cortical column: a structure without a function. Philosophical Transactions of the Royal Society of London B: Biological Sciences, 360(1456):837-862.

Hsu, C.-W. and Lin, C.-J. (2002). A comparison of methods for multiclass support vector machines. Neural Networks, IEEE Transactions on, 13(2):415425.

Kamitani, Y. and Tong, F. (2005). Decoding the visual and subjective contents of the human brain. Nature neuroscience, 8(5):679-685.

Kriegeskorte, N., Mur, M., and Bandettini, P. A. (2008). Representational similarity analysis-connecting the branches of systems neuroscience. Frontiers in systems neuroscience, 2:4.

Misaki, M., Kim, Y., Bandettini, P. A., and Kriegeskorte, N. (2010). Comparison of multivariate classifiers and response normalizations for patterninformation fMRI. NeuroImage, 53(1):103-118.

Moeller, S., Yacoub, E., Olman, C. A., Auerbach, E., Strupp, J., Harel, N., and Uğurbil, K. (2010). Multiband multislice GE-EPI at 7 tesla, with 16 -fold acceleration using partial parallel imaging with application to 
high spatial and temporal whole-brain fMRI. Magnetic Resonance in Medicine, 63(5):1144-1153.

Norman, K. A., Polyn, S. M., Detre, G. J., and Haxby, J. V. (2006). Beyond mind-reading: multi-voxel pattern analysis of fMRI data. Trends in cognitive sciences, 10(9):424-430.

Op de Beeck, H. P. (2010). Against hyperacuity in brain reading: spatial smoothing does not hurt multivariate fMRI analyses? NeuroImage, 49(3):1943-1948.

Rockland, K. S. (2010). Five points on columns. The neocortical column, page 6 .

Sasaki, Y., Rajimehr, R., Kim, B. W., Ekstrom, L. B., Vanduffel, W., and Tootell, R. B. (2006). The radial bias: a different slant on visual orientation sensitivity in human and nonhuman primates. Neuron, 51(5):661670.

Senden, M., Reithler, J., Gijsen, S., and Goebel, R. (2014). Evaluating population receptive field estimation frameworks in terms of robustness and reproducibility. PloS one, 9(12):e114054.

Sorger, B., Dahmen, B., Reithler, J., Gosseries, O., Maudoux, A., Laureys, S., and Goebel, R. (2009). Another kind of 'BOLD response': answering multiple-choice questions via online decoded single-trial brain signals. Progress in brain research, 177:275-292.

Sun, P., Gardner, J. L., Costagli, M., Ueno, K., Waggoner, R. A., Tanaka, K., and Cheng, K. (2012). Demonstration of tuning to stimulus orientation in the human visual cortex: a high-resolution fMRI study with a novel continuous and periodic stimulation paradigm. Cerebral Cortex, page bhs149.

Yacoub, E., Harel, N., and Uğurbil, K. (2008). High-field fMRI unveils orientation columns in humans. Proceedings of the National Academy of Sciences, 105(30):10607-10612. 
Yacoub, E., Shmuel, A., Logothetis, N., and Uğurbil, K. (2007). Robust detection of ocular dominance columns in humans using Hahn spin echo BOLD functional MRI at 7 Tesla. NeuroImage, 37(4):1161-1177.

Zimmermann, J., Goebel, R., De Martino, F., van de Moortele, P.-F., Feinberg, D., Adriany, G., Chaimow, D., Shmuel, A., Uğurbil, K., and Yacoub, E. (2011). Mapping the organization of axis of motion selective features in human area MT using high-field fMRI. PLoS One, 6(12):e28716. 

Chapter V

\section{General discussion}




\section{Histological validation of DTI tractography}

The aim of my first study was to validate fiber tractography results obtained from DTI measurements. To this end, we used histological tracers in a sample of human post-mortem tissue in order to obtain samples of connected brain locations. Following signal detection theory, we defined true and false positives and negatives based on the agreement between modeled and empirically obtained connections. From the ratio of the occurences of these cases, sensitivity (how likely is a real connection represented by a modeled one) and specificity (how likely are two unconnected points also unconnected by means of tractography) could be defined. We found sensitivity and specificity values of approximately $80 \%$ for one injection site which showed a pronounced unidirectional projection. For two other injection sites bearing more diffuse projection patterns, sensitivity and specificity were remarkably lower albeit still well above chance level. We saw these findings as evidence that DTI works as theoretically assumed: Unidirectional fiber pathways are described accurately, whereas multidirectional situations cannot be sufficiently modeled. In the latter case, sensitivity was affected more strongly, showing the inability of the tractography algorithm to construct a consistent pathway.

Besides the meaning of these findings for the assessment of DTI, we established a general method for comparing neuronal connectivity and possibly other characteristics between different types of anatomical data. In our case, the two sources of connectivity information (tracer vs tractography) differed largely in their spatial dimension (2D vs 3D), resolution (micrometers versus millimeters) and scale (individual axons vs major diffusivity), precluding direct comparisons. The signal detection approach we used allowed to overcome this obstacle by abstracting both data sources towards the contextually crucial information, the connectedness of pairs of locations.

Recently, this method has been adopted by a highly controversial paper (Thomas et al. 2014). The authors compare fiber pathways based on 
different dMRI models in the macaque brain to tracer data from a brain atlas. They report poor performance values (combined from sensitivity and specificity) and conclude that dMRI is severely limited in terms of anatomical accuracy. This interpretation highlights some of the covert pitfalls of the signal detection approach. The essential problem is that the observed sensitivity and specificity values are only partly an indicator of the discriminative power of the model. Another influencing factor is the difficulty of the signal detection "task", which in turn depends on a number of explicit and implicit parameters. Relevant factors in this case are for example the tolerance threshold applied in defining the injection site, the maximal considered distance from the injection site (the larger it is, the easier it is to score correct rejections since irrelevant locations enter the analysis) and the spatial scale on which the analysis is performed. What I see as the most important factor however, is the fact that in the study at hand, data from different individuals were compared which required an elaborate alignment process and certainly increased task difficulty.

Therefore, their findings might in fact not express the inability of diffusion MRI to correctly model fiber connections but rather show that the comparison procedure failed (to some extent at least, the reported results are still well above chance level in at least one of two analyzed ROIs). Reversely, this suggests of course that in our own study the task might have been too easy. However, the fact that we were able with the same procedure to obtain a very good performance in one case and a bad one in another case, indicates that our measures to balance task difficulty proved successful.

We can conclude that the most valuable information in signal detection analyses like the one we conducted does not so much lie in the absolute performance values but rather in their relations across analyzed cases. Therefore, the most important results of our study were the finding that DTI tractography works best in areas characterized by unidirectional fiber projections and the identification of an optimal FA threshold by means of optimizing the sensitivity/specifity tradeoff. This does not invalidate 
our findings of good absolute sensitivity and specificity values, but they should be seen as evidence rather than proof.

If the aim is to obtain an objective, absolute measure of the goodness of a method, one has to assess the difficulty to score correct and false judgements in the given context. Additionally, the different judgement categories (hit, miss, correct rejection, false alarm) might have to be evaluated differently: Tracers mark all fibers connected to the injection site. These, however, might represent only a small subset of the whole fiber population in a given ROI. Diffusion MRI tractography on the other hand models only sufficiently strong fiber pathways. Therefore, if a modeled tract projects to the injection site, we strongly expect to find a corresponding stained connection. Hits and false alarms in our paradigm are therefore a relatively reliable source of information. If on the contrary, a stained fiber tract is not reflected by a modeled fiber pathway, then this might simply mean that it was too small compared to more pronounced fiber paths. Hence misses and correct rejections are less significant for the absolute assessment of dMRI quality. These considerations should be taken into account when conducting or evaluating future studies following the principle we described.

\section{Histological validation of diffusion tensors}

In my second study, we investigated DTI on the level of diffusion tensors by means of a voxelwise comparison to myelin stained sections. We digitized these sections at microscopic resolution and determined image orientedness in each pixel following a structure tensor paradigm. Based on a precise alignment of both data sources, we mapped dMRI voxels to histological image compartments of the same size. For these image parts, we determined orientation distributions and fitted them with von Mises distributions. This yielded averaged fiber orientation values, which were comparable to the orientations of the diffusion tensors after projection into the sectioning plane. 
In white matter, we found an overwhelming agreement between DTI and the myelin staining in the presence of unidirectional fiber pathways, with the majority of voxels showing less than $10^{\circ}$ deviation. We were also able to confirm the weakness of DTI when facing fiber crossings. We found that in this case, diffusion tensor directions tend to lie in between both fiber directions. Taken together, this convincingly demonstrates the correctness of the well-established theoretical assumptions about DTI.

Surpassing previous expectations, we found a remarkable goodnessof-fit even in the gray matter up to layers III/IV. In the deeper layers V and VI, the match between diffusion tensor and myelin orientations was not considerably worse than in white matter. This showed that, given the high resolution of our scan, DTI is a suitable method to model parts of gray matter, particularly cortical insertions of fiber tracts. Since in gray matter, FA is lower than in white matter, we discussed an adaption of tractography parameters when modeling fiber pathways across the white matter - gray matter boundary. In the middle layers III and IV, DTI seemed to robustly follow structures which were invisible to our histological method, resulting in a mismatch much larger than what is explainable by chance. We suggested unmyelinated apical dendrites to be the source of this systematic mismatch and speculated about alternative histological approaches to further investigate this situation.

A possible follow-up to this study would be to compare complex dMRI models and DTI at a relatively higher spatial resolution in a similar validation study. This could provide useful information regarding the open question which is the better path to take with advancing MRI hardware: Employing more powerful models, or sticking to the simple model, yet with better imaging parameters. Since our histological analysis produces empirical fiber orientation distributions for arbitrarily sized image compartments, it theoretically allows for such a comparison.

However, there will be practical challenges to solve: First, a quantitative measure to compare the goodness-of-fit of different models at different resolutions has to be found. With models reflecting the entire FOD, a 
simple comparison of primary orientations would carry their purpose ad absurdum. One idea would be to extrapolate a diffusion tensor towards a simple synthetic FOD, for example a cyclical normal distribution where the primary orientation provides the mean and the fractional anisotropy the spread. From there, a comparison of goodness-of-fit values between modeled and empirical FODs might be reasonable.

The most important point though is the suitability of the histological data given their limited quality. Like all empirical data, the microscopic section images we obtained were noisy, both due to the process of digitization and the stained fiber material itself. This made it necessary to pool over large numbers of image pixels. In our case, $254 \times 254$ pixels were the equivalent to one imaging voxel, which was sufficient to provide robust FODs. A tentative reduction of the resolution to half the voxel size already revealed substantially less robust histological orientation estimates. Therefore, in order to extend our analysis towards finer resolutions or whole FODs, an exceptional histological quality is a prerequisite. With Gallyas stains however, the practical limitations might already have been reached in our study since at very large magnification factors, individual silver grains become apparent, physically "pixelating" the histological image. This underlines the already discussed notion that there might be a better suited staining method to proceed with this validation approach.

\section{Intermediate conclusion}

Both DTI validation studies taken together, we showed that DTI can reliably model nerve fibers both within and across voxels. The precondition is an underlying fiber architecture which can be expressed within the limited framework of the model. Our findings had already been theoretically assumed and partly been demonstrated in phantoms and animal studies. With our work, we closed a gap where direct comparisons between DTI data and human brain tissue was missing. Future studies might extend this work towards a more profound analysis of DTI in gray matter and 
an investigation of more complex dMRI models. The question for the anatomical underpinnings of DTI in white matter however, we can now largely regard as solved.

\section{Radial bias in fMRI orientation decoding}

My third study was concerned with the radial bias as evident in $7 \mathrm{~T}$ fMRI data of the early to mid ventral processing stream. Particularly, we aimed to investigate its role in orientation decoding by means of pattern classification. In a preparatory experiment, we used population receptive field mapping to localize visual cortices V1 to V4 and to map the polar positions of voxel receptive field centers. In the main experiment, subjects viewed flickering oriented bar patterns. The BOLD responses towards the different stimulus orientations were analyzed both classically by means of linear regression and by discrimination of the respective voxel patterns through support vector machines.

We were able to replicate earlier findings that a radial bias is present in fMRI data and that viewed orientation can be reliably decoded. Evaluating the influence of individual factors such as radial bias towards decoding performance is generally difficult due to the relative inaccessibility of the inner workings of most classification algorithms. One reported approach is to bin data according to a certain pattern, normalize them within bins and assess the effect on decoding performance. We argue that this is only conclusive in the case where the performance is impaired by the normalization, whereas the absence of an effect is difficult to interpret. We illustrated this argument with simulated data and proposed an extended version of the normalization approach with a series of increasing bin counts and different shapes of bin patterns. However, even with this method, we were not able to affect the classification.

An alternative approach is to perform classification separately for individual data bins. Doing so, we could show successful orientation decoding on circular segments of the visual field, albeit at slightly decreased perfor- 
mance. We conclude that the radial bias is involved in the classification process, but does not play a pivotal role.

This finding implies the question what kind of pattern is mainly responsible for orientation decodability and on what spatial scale this pattern resides. We cannot answer this question based on our data but as discussed in chapter 4, compelling arguments against subvoxel influences lead us to regard a mesoscale pattern as the main contributor. This assumption is supported by visual inspection of cortical surface maps of orientation preference and SVM weights, whose spatial frequency is on the scale of patches of voxels rather than individual ones.

To go forward in the investigation of multi-voxel pattern analyses, we need better approaches to test their informational basis. To this end, the approach of trying to distort classifier results might pose a dead end. First, classifiers seem in general to be too robust to be susceptible to this kind of examination. Second, classification performance as a function of the amount of predictive information present and the SNR is not well-studied. We do not know how smoothly or abruptly classification performance decreases upon gradual removal of crucial information, likewise we cannot objectively interpret the amounts of performance loss caused by a certain manipulation.

Consequently, one possible direction of future research would be to perform extensive simulation studies in order to obtain a better understanding of how classification performance is related to SNR levels, different types of effects, data properties such as autocorrelations etc. The data simulation could be either simplistic, solely focussed on the studied properties, or it could attempt to simulate fMRI data, as for example in Chaimow et al. (2011). Another promising path to take is to apply our analyses to the output of artifical vision models. This could prove useful in investigating both the sources of orientation decoding and the principal behavior of MVPA. One example of such a study is the already addressed work by Carlson (2014), but there are many more models worth exploring (Olshausen and Field 1997, Serre et al. 2007). The advantage of this approach 
is that it can be controlled which information is present in the generated data and to which extent while at the same time these data are supposed to reflect (aspects of) the real processes leading to fMRI data. However, comparisons between fMRI and modeled data are usually made on the level of the final results, e.g. orientation preference or classification performance in our case. Consequently, this approach is a very indirect one and showing the same pattern of results does not necessarily mean that a model correctly reflects brain activity. Therefore, results gained from these kinds of analyses have to be interpreted with modesty.

\section{Conclusion}

The field of magnetic resonance imaging is steadily growing and advancing, both in the structural and the functional domain. Over the last decades, we have seen great advances in MRI hardware with ultra-high-field MRI becoming accessible as a research tool for neuroscientists and psychologists. Advanced hardware, however, has to go along with advanced methods. For this reason, new models and techniques are constantly being developed in order to improve MRI acquisitions and to obtain new kinds of information. An important part of this progress is the validation of models, leading to their establishment, improvement, or abandoning. With my work on diffusion tensor imaging and multi-voxel pattern analyses, I hope to have made a valuable contribution towards a more complete understanding of these methods.

\section{References}

Carlson, T. A. (2014). Orientation decoding in human visual cortex: new insights from an unbiased perspective. The Journal of Neuroscience, 34(24):8373-8383.

Chaimow, D., Yacoub, E., Ugurbil, K., and Shmuel, A. (2011). Modeling and 
analysis of mechanisms underlying fMRI-based decoding of information conveyed in cortical columns. NeuroImage, 56(2):627-642.

Olshausen, B. A. and Field, D. J. (1997). Sparse coding with an overcomplete basis set: A strategy employed by V1? Vision research, 37(23):33113325.

Serre, T., Oliva, A., and Poggio, T. (2007). A feedforward architecture accounts for rapid categorization. Proceedings of the National Academy of Sciences, 104(15):6424-6429.

Thomas, C., Frank, Q. Y., Irfanoglu, M. O., Modi, P., Saleem, K. S., Leopold, D. A., and Pierpaoli, C. (2014). Anatomical accuracy of brain connections derived from diffusion MRI tractography is inherently limited. Proceedings of the National Academy of Sciences, 111(46):16574-16579. 
Appendix A

\section{Knowledge valorization}


As I see it, there are three main motivations for us neuroscientists to study the brain: First, there is a philosophical aspect to it. For hundreds and thousands of years, great thinkers have been occupied with various existential questions. Historically, the authorities in answering those have been philosophers, shamans and priests. Modestly starting at the time of the renaissance and with ever increasing pace since then, the sciences have become another major authority. Nowadays, physicists for example develop theories about the origins of the universe and biologists about the genesis of life and mankind. Neuroscience in this regard is often concerned with questions related to the mind, such as how consciousness emerges and whether or not we have a "free will" (whatever this term means). Such questions are extremely hard to answer and my intention is not to say that science is particularly successful in doing so. However, humans crave to find the meaning of life and against this background, even incomplete, suboptimal or simply wrong answers can be more satisfying than not having an answer at all. Therefore, the pure attempt to solve the mysteries of our existence in a professional manner bears some societal value.

Second, and more importantly, there is the vast field of clinical applications of neuroscience. As mentioned in my introduction, MRI has been developed as a diagnostic tool, initially with the intention to detect tumors in regions which are otherwise hard to investigate. Even though nowadays MRI, particularly functional MRI, has many research applications without a clinical context as well, in its core it has kept this role. Improving the possibilities to diagnose pathological conditions in a fast, noninvasive way is without any doubt a significant achievement for our society. Aside of that, the health system is a huge economic sector. Therefore, neurodiagnostic methods certainly have a considerable economic value, too.

In this regard, for an imaging method to actually become a clinically applicable tool, several criteria have to be met: First of all, it has to be valid, providing robust and meaningful information about the examined tissue. Second, the method has to be sufficiently operable. This means there have to be a set of reasonably standardized parameters which allow 
users with only basic knowledge about its theoretical background to obtain good results. Finally, the interpretation of the results has to be clear, it has to be precisely known what information is contained in the acquired image volumes. These requirements cannot be guaranteed solely based on theories, but it has to be investigated in practice whether a method lives up to its expectations. Thus, making an imaging method good not only requires research and development, but also quality control, i.e. validation. This is precisely what I was concerned with in the first pillar of my doctorate, the validation of diffusion MRI. I showed first of all that a particular method of dMRI - DTI - works as it was theoretically assumed to do. I also helped towards optimizing model parameters and pointed out future directions to enhance the scope of this method. Therefore, I hope that this part of my work will play a role in the further development of dMRI and facilitate its establishment as a widely used clinical tool.

It also has to be mentioned that the clinical applications of neuroscience are not limited to only diagnostics anymore. For quite a few diseases of the brain or the mind, therapies based on neuroscience findings have been developed over the last decades. A prominent example of this is deep brain stimulation, which has proven helpful for example in cases of Parkinson's disease and major depression.

Third, there is the field of computational neuroscience. There have been many analogies for brain activity in the past. While the ancient Greeks thought of the brain as a blood cooler, in modern times the crucial link between its activity and our mind was recognized and people started to think of the brain as a steam engine, clockwork or whatever the cutting-edge machine of their time was. Today, the brain is most often seen in analogy to a computer. As such, it might not be the most powerful exemplar in terms of hardware specifications like processing speed or storage space (although this is hard to quantify). But qualitatively, the brain has some very powerful features, most notably being intelligent and able to learn. Particularly intelligence, which might be described as a set of very effective heuristics, allowing us to quickly find solutions to any kinds of new problems, is something which has not remotely been 
achievable with computers so far. Therefore, computational models of how the brain works can inspire software development, especially into the direction of artificial intelligence. In recent years, we have seen this in the example of so-called deep learning. Essentially a resurgence of old neuronal network paradigms, combined with state-of-the-art processing power, these networks show an unprecedented level of classification performance, being able to distinguish between various seen objects, faces, or heard words. These programs are still quite "dumb" in an AI sense, effectively doing little more than extracting patterns from huge data sets. However, doing this well enough to beat for example grandmaster Go players (something which some years ago was regarded as impossible), deep learning networks already show a vague resemblance of intelligence and are certainly a promising step towards a true AI.

What might sound like a rather playful field of application has in fact both a huge impact on our society and an equally huge economical potential. Many big companies have over the last decade started to acquire enormous sets of data about their (factual or potential) customers, such as which websites they visit, where they are at certain times of the day or what they like on social networks. States' security services gather similar data and store who we are talking to and when with the aim to anticipate terrorist attacks. Their main problem though is that they often lack the methods to even rudimentarily analyze all the collected data and here, the potential of semi-intelligent, neuronally inspired pattern classifiers starts to be fully recognized. I want to point out that this is a very delicate matter since our society might at the moment stand at a crossroads. With ever growing capabilities to infer various facts from seemingly innocuous personal data, the question which data we allow to be collected and what to be done with them might determine which world we live in tomorrow. Will it be comparable to how we live today, or are we going to be automatically classified on a wide range of dimensions, such as professional aptitude, healthiness or psychological fitness, by our governments and, possibly worse, all kinds of companies? Are you and me going to be able to apply for the same jobs and buy the same 
products at the same prices, or will this depend on the output of algorithms determining how likely we are to succeed and how much we would be willing to pay? We are already seeing big steps into the second direction today and I am sometimes a little concerned about the future development. The more severe the responsibility of researchers is who develop and improve classifiers. They should not be blind to how their work is utilized and make their efforts depend on the continuous assessment whether their inventions will rather help us or put us in self-made chains.

Another practical application of the brain-computer analogy is to develop actual brain-computer-interfaces, which can work in two directions: One can transform digital data into nerve stimulation, as it is done with retina and cochlea implants. Or one can acquire brain activity data (such as fMRI data) and classify them in real time. This is very interesting in several clinical contexts. For example, BCIs of this kind could allow locked-in patients to communicate at least their basic needs with others, potentially increasing their quality of life by a large margin. Also, a new device to translate trained neuronal output into muscle stimulation has recently been successfully tested in a paraplegic patient, giving him back the ability to move his hand. So far, the practicability and efficiency of BCIs is still very limited. They require data acquisition techniques such as fMRI scans or permanent electrode implants which are very cumbersome from the perspective of daily life, and the amount of information which can be transmitted in a given time is very limited. For this reason, BCIs are contemporarily worthwile mostly as technical aids for people who have no other means of signal transmission. However, should this technology advance towards a level where operating machines cognitively becomes more comfortable than by using keyboards etc., BCIs could see a much wider range of application, completely revolutionizing the way people work and spend their leisure time.

My fMRI study, described in chapter 4, is a piece of fundamental research which aims to provide basic knowledge for both branches of computational neuroscience outlined above. With regard to the first part, I described and tested methods aimed to analyze the inner working of clas- 
sifiers and eventually come to a better understanding of their performance. With regard to the second part, I used classifiers to decode visual stimuli from brain activity, and I enhanced the classification capabilities described in earlier studies by demonstrating decodability on small subsets of the visual cortex. While this study might be too methodologically focused to be directly translatable into concrete products, I hope to have convincingly argued that studies like this are the cornerstones of invaluable future developments.

Let me finally say that with the simplifying view brought about in this section, essentially reducing neuroscience to three subfields, I certainly forgot branches of neuroscience which might be just as valuable for society and economically profitable. I therefore want to stress that my intention is not to belittle any left-out branches but rather to show the most important reasons which I personally identified why we as a society should spend ressources into neuroscientific research. 
Appendix B

Acknowledgements 
I do not want at this point to list all people I know, but I also do not want to order them by how much I like them and then you might be the first one who did not make it onto the list. Therefore, without naming anyone, let me say thank you to all those who helped me factually or figuratively at creating this piece of work.

One exception though, since you honestly did not really help me at all with my work: Thank you for being here, Melis, I love you!

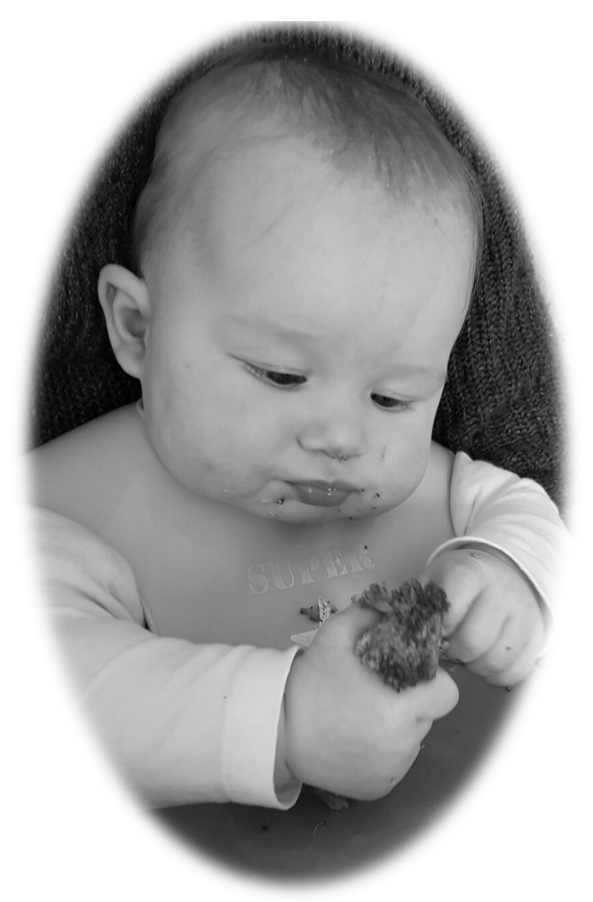


Appendix C

\section{Curriculum vitae}


Arne Seehaus was born on the 17th of June 1985 in Heppenheim, Germany. He started his school education in 1991 at the Schloss-Schule, a European school with a focus on intercultural exchange and understanding. In 1995, he went on to high school at the Altes Kurfürstliches Gymnasium in Bensheim. Here, he obtained a humanist education garnished with ample lessons in Latin and ancient Greek. He left high school in 2004 with an excellent graduation and a wide range of interests to pursue in his following studies. In winter semester 2004/2005, Arne enrolled at the Technische Universität Darmstadt in Mathematics, soon after followed by Psychology as a second major. In 2009, he started his doctorate studies. After a short period of activity in the field of work psychology under Prof. J. Vogt, he moved to the field of neuroscience in a joint project between TU Darmstadt (Prof. R. Galuske) and Maastricht University (Prof. R. Goebel). In Maastricht, Arne earned his doctorate in 2016. 